\title{
Abstracts presented at the 14th European Colorectal Congress (\#ECCStGallen), 29.11.2020-2.12.2020, St.Gallen, Switzerland
}

\section{Michel Adamina •}

${ }^{1}$ Department of Surgery, Kantonsspital Winterthur, 8401 Winterthur, Switzerland

${ }^{2}$ Faculty of Medicine, University of Basel, Basel, Switzerland

${ }^{3}$ Department of Surgery, University of Trieste, Trieste, Italy

(C) Springer Nature Switzerland AG 2021

The 14th European Colorectal Congress of St.Gallen took place from Sunday 30.11.2020 to Wednesday 2.12.2020. A total of 1403 registered participants from 81 countries enjoyed 50 Expert Lectures for another successful \#ColorectalSurgeryWeek. One in twelve participants submitted an abstract and 143 papers out of 178 submissions were selected for digital presentation at the congress, including a virtual discussion.

The abstracts of all presented posters are printed below. Of those, an international jury made of Michel Adamina, Winterthur- $\mathrm{CH}$;
Alberto Arezzo, Torino-IT; Christianne Buskens, Amsterdam-NL; Dieter Hahnloser, Lausanne-CH and Gianluca Pellino, Barcelona-SP had the difficult task to select the best contribution.

The Poster Prize of EUR 10,000 was awarded to Dr. Pankaj Kumar from Bhubaneswar, India for his work on "LIFT vs. ERAF in patients with fistula in ano-an interim analysis of the FRAIL randomised controlled trial. Congratulations to all abstract authors, in particular to the winner team from the All India Institute of Medical Sciences.

\section{Correspondence Author}

Michel Adamina, MD, PD, MSc, EMBA HSG, FEBS, FASCRS

Chief of Colorectal Surgery

Departement of Surgery

Kantonsspital Winterthur

8401 Winterthur

Switzerland

Tel. +4152 2663376

michel.adamina@gmail.com

www.colorectalsurgery.eu

@MichelAdamina
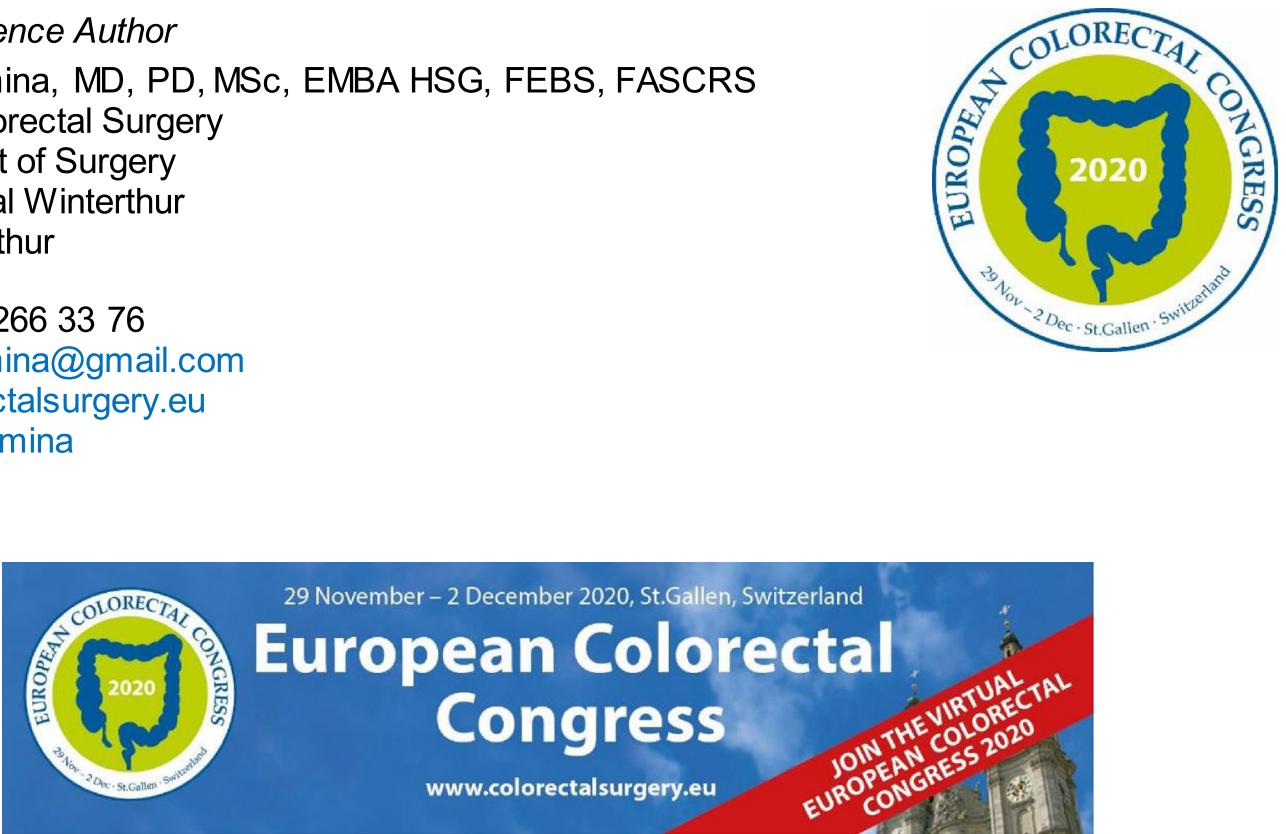


\section{REVERSAL OF LOOP ILEOSTOMY IN RECTAL CANCER PATIENTS-A SINGLE CENTRE 5 YEAR REVIEW}

\author{
Lara Armstrong, Catherine Rossborough, Kevin McElvanna \\ Craigavon Area Hospital, Craigavon, GB
}

Background/Aim Formation of a de-functioning loop ileostomy following an anterior resection (ARLI) allows proximal diversion of faecal material, therefore limiting the consequences in the event of anastomotic failure. Earlier closure has been suggested to reduce stoma-related morbidity, improve quality of life whilst effectively protecting the distal anastomosis. Our aim was to determine if reversal rate of ARLI is comparable to current national 18 months stoma standards-UK National Bowel Cancer Audit reports. Methods We reviewed the time interval to reversal of ARLI, from 2013 to 2018 in a single colorectal unit. Age, gender, date of initial surgery, date of reversal, stoma related admissions and reason for possible delay to reversal (6 months) were recorded. Results 50 patients underwent ARLI for rectal cancer between 2013 and 2018, 72\% male, median age 66 (ranging 33-83). 42 patients underwent an 18 month follow up period, $40.5 \%$ of which still had an ileostomy. Median time to reversal 9 months (3-37). $37.3 \%$ received post-operative chemotherapy and $30 \%$ required admission due to stoma morbidity. Conclusion A higher 18-month stoma rate in ARLI patients, compared with UK National Bowel Cancer Audit Reports (35\% 18 month stoma rates). Prolonged reversal time was expectedly observed with advanced stage. Reason for delay included pending water soluble results, adjuvant chemotherapy and patient morbidity.

\section{THE IMPACT OF THE ADVANCED MULTIDISCIPLINARY TEAM (MDT) WORKING ON STAGE IV COLORECTAL CANCER}

\section{Elroy Weledji \\ University of Buea, Buea, CM}

Background/Aim The management of stage IV colorectal cancer is optimized by bringing together all relevant specialties in colorectal metastatic (CRLM) disease management in a centralized high volume liver cancer surgery center. The major objective is to increase respectability so as to achieve long-term potential survival ( $>5$ years). New chemotherapy regimens including biologicals are bringing more patients to resection. Including respectable extrahepatic disease, respectability is the complete removal of liver metastasis while leaving at least $30 \%$ of functional remnant liver. The aim of the study was to evaluate the impact of the objectives of the advanced MDT on the clinical outcome of Stage IV colorectal cancer. Methods A British Association of Surgical Oncology (BASO) Ronald Raven traveling fellow '2 week' observational study on the role of the advanced MDT in the management of stage IV colorectal cancer at the Aintree hepatobiliary center, Liverpool UK. Results The major end point of the advanced multidisciplinary approach to stage IV colorectal cancer management is resectability due to an impact on patient survival of $40 \%>5$ years and $30 \%$ in 10 years. There are 3 categories of patients with resectable disease: (1) $10-20 \%$ of patients with liver-limited disease are resectable with curative intent at time of detection (i.e. easily resectable), (2) $10 \%$ are amenable to surgical intervention-resection with ablation or two stage hepatectomy with concomitant resectable extrahepatic disease (i.e. borderline resectable), (3) $30-40 \%$ of patients with initially liver-limited irresectable disease are made resectable by chemo/ biological therapy.
Thus, $50-70 \%$ of CRLM are potentially resectable. Conclusion The overall survival from medical and surgical treatment of CRLM increased significantly from $30 \%$ in 2008 to $70 \%$ in 2011 with the advent of the advanced MDT in Aintree, Liverpool, UK. The nonadherence to MDT decisions leads to lower survival rates.

\section{IMPACT OF OBSIDIAN ${ }^{\circledR}$ ASG AUTOLOGOUS PLATELET-RICH FIBRIN MATRIX ON ANASTOMOTIC HEALING IN COLORECTAL SURGERY}

Andreas Shamiyeh, Bettina Klugsberger, Carina Zeitlhofer, Wolfgang Schimetta, Friedrich Herbst, Bernhard Dauser

Kepler University Clinic, Linz-AU

Background/Aim The use of regenerative medicine in colorectal surgery constitutes an entirely new therapeutic principle. The aim of this new therapeutic approach is to reduce the anastomotic leak rate and minimise morbidity and mortality. The literature identifies the leak rate for colorectal operations as $3-39 \%$. Methods This is a prospective, multi-centre descriptive study commencing in June 2018. As part of the elective laparoscopic colorectal surgery, an autologous fibrin matrix was used as part of anastomotic technique in conjunction with activated thrombocytes (Obsidian $\mathrm{ASG}^{\circledR}$ ). During anastomosis, this matrix was applied after resection onto the colorectal tissue surfaces with the aim of triggering tissue regeneration and improved wound healing. The anastomotic leak rate was primary outcome parameter. Secondary outcomes were faecal blood, fever and the length of the hospital stay. Results Between 01/07/2018 and 31/12/ 2019, 101 elective laparoscopic procedures were conducted: 78 for sigmoid resection and 23 for rectum resection, for either benign or malignant disease. The study included 51 women and 50 men. The average age of the patients was $63.6( \pm 11.6)$ years. Apart from a left hemicolectomy, all operations were carried out with laparoscopic assistance. Postoperatively, 11 patients $(11 \%)$ presented with postoperative faecal blood without an associated anastomotic leak: 2 patients experienced an anastomotic leak $(2 \%)$, one rectum $(4.3 \%)$, one sigmoid $(1.3 \%)$. None of the patients suffered undesirable side effects due to the application of Obsidian ASG. None of the patients died. Conclusion The therapeutic concept of tissue regeneration appears to be a promising approach to further reduce the anastomotic leak rate. Further randomised controlled studies are required to review the statistical significance of these results.

\section{ASSOCIATION BETWEEN INTRAOPERATIVE BLOOD GLUCOSE AND ANASTOMOTIC LEAKAGE IN COLORECTAL SURGERY}

Murièl Reudink Máxima, Daitlin Huisman, Louis Lieverse, Stefan van Rooijen, Rudi Roumen, Freek Daams, Gerrit D Slooter

Veldhoven, $N L$

Background/Aim Perioperative hyperglycaemia is a known risk factor for postoperative complications after colorectal surgery. The aim of this study was to investigate whether values of intraoperative blood glucose (BG), obtained at the moment of anastomosis creation, were associated with colorectal anastomotic leakage (CAL) in diabetic and non-diabetic patients undergoing colorectal surgery. Methods The present study is a subgroup analysis of the previously published LekCheck study enrolling consecutive adult patients 
undergoing colorectal surgery with primary anastomosis between 2016 and 2018. From all included patients preoperative diabetes status was known and intraoperative BG was determined. Primary outcome was the occurrence of CAL within 30 days postoperatively. Results Of 1474 patients (mean age 68 years), 224 patients (15\%) had diabetes mellitus, 737 patients $(50 \%)$ presented with intraoperative hyperglycaemia $(\geq 126 \mathrm{mmol} / \mathrm{dL},>7.0 \mathrm{mmol} / \mathrm{L})$, and 129 patients $(8.8 \%)$ developed CAL. Patients with intraoperative hyperglycaemia had significantly higher CAL rates compared to patients with a normal glucose level (12\% versus $5 \%, \mathrm{P}<0.001)$. Considering non-diabetic patients, a glucose level of $126-200 \mathrm{mg} / \mathrm{dL}$ (7.0-11.1 $\mathrm{mmol} / \mathrm{L}$ ) was associated with an increased CAL risk (OR 4.87) as was a glucose level of $>200 \mathrm{mg} / \mathrm{dL}(>11.1 \mathrm{mmol} / \mathrm{L})(\mathrm{OR}$ 9.11). By contrast, this increase in risk was not seen in diabetic patients. Conclusion Incidence and severity of intraoperative hyperglycaemia are associated with CAL in non-diabetic patients but not in known diabetic patients. Whether hyperglycaemia is an epiphenomenon or a marker for other risk factors or a modifiable risk factor per se for CAL requires future research.

\section{A PILOT AUDIT OF NEGATIVE APPENDICECTOMY RATE IN A SINGLE SCOTTISH INSTITUTION}

\section{Elizabeth Westwood, Susan Moug, Carly Bisset Paisley}

\section{GG Royal Alexandra Hospital}

Background/Aim The Right Iliac Fossa Pain Treatment (RIFT) Study found a negative appendicectomy rate (NAR) of $20 \%$, in patients aged $16-45$ in the UK-far higher than other countries. Our aims were two-fold: firstly; to determine our institution's negative appendicectomy rate, and secondly; to identify strategies which may minimise the risk of unnecessary appendicectomy. Methods We performed a retrospective audit over 6 months (July 2019-Jan 2020). Patients aged over 16 years who had the following codes: "Laparoscopic Appendicectomy", "Open Appendicectomy" or "Diagnostic Laparoscopy" ( $=68$ ) were identified from the emergency theatre logbook in a single institution, which were then crossreferenced with electronic records. This allowed exclusion of cases where the appendix was not removed for appendicitis $(n=5)$, or confirmation was not possible $(n=4)$. Index diagnostic suspicion of appendicitis, inflammatory markers, imaging and histological diagnosis were evaluated. Results In a 6 month period, 59 patients underwent appendicectomy: $51 \%$ female, $49 \%$ male. Median age was 43 years (range 17-79) with appendicitis suspected at admission in $81 \%$. Mean admission WCC was $14.4($ norm $<10)$ and median CRP was 47 (norm < 10). $32 \%$ of patients had either normal WCC or CRP (17\% normal WCC, $19 \%$ normal CRP, $3 \%$ both markers normal). $69 \%(n=41)$ of patients had pre-operative imaging; CT was the commonest modality-37/41 scanned patients. Radiological evidence of appendicitis was found in $92 \%$ of imaged patients. The institution's NAR was $8.5 \%$ (10\% and $6.9 \%$ for females and males respectively). NAR among non-imaged patients was $22.2 \%$ versus $2.7 \%$ in imaged patients. Conclusion Our institution's NAR was significantly lower than the UK average. Lower NAR among imaged patients highlights the importance of early imaging in accurate diagnosis, regardless of gender. We believe low scanning thresholds, aided by good working relationships with radiology, contribute to better than average national NAR. Access to short-term follow-up via a consultant-led "Hot Clinic" service offers further safety netting, and may increase clinical confidence in conservative management or observation in low risk patients.

\section{LONG-TERM OUTCOME OF RADICAL EXCISION VS. PHENOLISATION OF THE SINUS TRACT IN PRIMARY SACROCOCCYGEAL PILONIDAL SINUS DISEASE; A RANDOMIZED CONTROLLED TRIAL}

Akke Pronk, Michiel Vissink, Niels Smakman, Edgar Furnee

Groningen, NL University Medical Cent Groningen

Background/Aim The aim of this randomized trial was to compare the long-term outcome between pit excision and phenolisation of the sinus tracts vs. radical excision with primary wound closure as treatment for sacrococcygeal pilonidal sinus disease (SPSD). Methods A total of 96 patients with primary SPSD were randomized; 50 patients underwent phenolisation and 46 radical excision. Long-term outcome was obtained by an online questionnaire, including quality of live (Short-Form 36), recurrence of SPSD and SPSD-related complaints. Results A total of 74 patients $(77.1 \%)$ completed the questionnaire; 36 patients after phenolisation and 38 after excision. The mean $( \pm \mathrm{SD})$ time to follow-up was $48.4( \pm 12.8)$ and 47.8 $( \pm 13.5)$ months, respectively. There was no significant difference between both group with regard to quality of life and SPSD-related symptoms. In the phenolisation group, two patients (5.6\%) developed a recurrence and one patient $(2.6 \%)$ in the excision group $(\mathrm{P}=0.604)$. Five patients $(13.9 \%)$ in the phenolisation group required a second phenolisation treatment to reach complete wound healing. The impact of treatment was significantly less after phenolisation $(\mathrm{P}=0.010)$. Conclusion Recurrence rate and quality of life were not significantly different between phenolisation and radical excision for primary SPSD after a follow-up of 4 years. The impact of the whole treatment was significantly less after phenolisation, however, approximately $14 \%$ of patients required a second phenolisation treatment to reach complete wound healing.

\section{ROBOTIC ASSISTED ULTRA-LOW ANTERIOR RESECTION USING TRANSANAL PLATFORM FOR LOW RECTAL CANCER}

Marcos Carvajal, Misael Ocares, Christian König, Jose Vivanco, Gino Caselli, Claudio Benavides

Conception, $C L$

Background/Aim Robotic assisted rectal resections make sense within the narrow confines of the pelvis and several studies have shown reduced rates of conversion to open and less blood loss compared to laparoscopic approach (S. Lee et al. Int J Colorectal Dis 2018). In addition, the transanal platform (GelPoint Path) allows a better exposure of the distal rectum and direct determination of distal resection margin (Yao et al. Surg Innov. 2020). Methods A 65-yearold female patient with low rectal cancer $5.7 \mathrm{~cm}$ cT3bN2M0, two centimeter over puborectalis muscle with total neoadjuvant chemoradiotherapy underwent a robotic assisted ultralow anterior resection using Da Vinci X? system and the distal resection margin using transanal platform (GelPoint Path?). Bowel perfusion was assessed prior to fashioning the anastomosis using indocyanine green (ICG) $0.5 \mathrm{mg} / \mathrm{ml} / \mathrm{kg}$ i.v. Results Robotic console was docked at left oblique position for abdominal and rectal phase, and there was no need for table repositioning (redocking). The inferior mesenteric artery was identified and ligated. Followed by medial to lateral approach, left ureter and gonadal vassels were respected. Subsequently, through the avascular plane, mesorectum was dissected totally down to the level of the pelvic floor. Then, transanal approach 
was performed with GelPoint Path? With insufflation stabilization bag (ISB). A purse-string suture is placed $1 \mathrm{~cm}$ distal to the tumor. A fullthickness incision of rectal wall is performed circumferentially using ultrasonic dissection. We marked the intended level of transection of the proximal colon and the anesthesiologist was instructed to administer $0.5 \mathrm{mg} / \mathrm{mg} / \mathrm{kg}$ of ICG intravenously. Two minutes later, the demarcation line of fluorescence matched the marked level of intended bowel transection. Once the specimen has been removed, coloanal end-to-end hand-sewn anastomosis plus protective loop ileostomy are performed. The postoperative course was uneventful. The patient was discharged 5 days after surgery. Pathological biopsy indicated ypT2NOM0 with tumor-free resection margins. Conclusion The transanal approach to determine the distal resection margin is an easy, feasible and safe method compared to the transabdominal approach in robotic assisted ultra-low anterior rectal resection.

\section{A REVIEW OF MICROSATELLITE INSTABILITY (MSI) TESTING: REAL WORLD CLINICAL DATA}

\section{Chad Harris}

\section{Newcastle, GB, Northumbria Health Care NHS Trust}

Background/Aim Colorectal cancers (CRC) are the second most common cause of death by cancer, in the UK. Microsatellite instability (MSI) analysis is novel yet important part of managing CRC and as a tool for predicting prognosis, treatment and identify lynch syndrome. Where lynch syndrome is identified, preventative screening can be utilised. Previous studies have only focused tumor testing in high risk cases. Methods A retrospective study at Northumbria Health Care NHS Trust was performed on all new CRC patients who underwent MSI analysis between 2017 and 2020. Results A total of 965 patients with CRC were identified. After exclusion criteria was applied to the cohort, a total of 483 patients were identified as having undergone MSI analysis. The mean age was 73.5 years old, with the female to male ratio being $1: 1.4$. Patients were further grouped into MSI stable, low and high. MSI High patients accounted for $10 \%$ of patients analysed. Further genetic testing was performed on these patients which highlighted 28 patients with BRAF positive genes who went on to screening for Lynch syndrome associated cancers. Conclusion MSI testing can provide essential diagnostic and prognostic information and also guide treatment options. $2.9 \%$ of patients were identified as high risk for familial cancers and went on to have further genetic and surveillance screening.

\section{THE ROLE OF PRO-INFLAMMATORY CYTOKINE AND ADIPOKINE PROFILES IN PATHOGENESIS OF CRYPTOGLANDULAR PERIANAL FISTULA}

\section{Marcin Wodarczyk, Jakub Fichna \\ Medical University of Lodz, Lodz, PL}

Background/Aim This prospective study aimed at evaluating the role of hormonal activity of adipose tissue and pro-inflammatory cytokine profile in the pathogenesis of cryptoglandular perianal fistulas. Methods In the study 80 patients (46 male, 34 female) who were hospitalized in the Department of General and Colorectal Surgery at the Medical University of Lodz and underwent surgery due to cryptoglandular perianal fistula have been enrolled. Levels of adipokines: adiponectin, leptin, resistin, IL-1, IL-8, IL-12 and TNF- $\alpha$ in perianal tissue and in serum were determined. Results The expression of adiponectin, leptin and resistin were dedected in $91 \% ; 84 \% ; 81 \%$ of canal tissue in the anal fistulas. Also, cytokines IL-1, IL-8, IL-12 and TNF- $\alpha$ were present in respectively, 73, 78, 81 and $83 \%$ of the anal fistulas. The tissue levels of adiponectin, leptin and resistin positively corelated with BMI. Complex fistula (defined as fistula with $>30 \%$ of external sphincter involvement, or anterior fistulas in female patients, as well as recurrent fistulas) was found in $41 \%$ of obese patients vs. $11 \%$ in non-obese patients $(p=0.008)$. Complex, in compare to simple fistulas, was related with signifcantly decreased levels of adiponectin $(4998 \pm 241$ vs. $6998 \pm 341 \mathrm{ng} / \mathrm{mL}$, $\mathrm{p}=0.012$ ) and eleveted levels of resistin, IL-1? And TNF-a (respectively, $16.1 \pm 2.1$ vs. $13.6 \pm 0.9 \mathrm{ng} / \mathrm{mL}, \mathrm{p}=0.032 ; 1.91 \pm 0.13$ vs. $1.63 \pm 0.18 \mathrm{pg} / \mathrm{mL}, \mathrm{p}=0.041 ; 15.5 \pm 3.9 \mathrm{vs} .19 .8 \pm 4.3 \mathrm{pg} / \mathrm{mL}$, $\mathrm{p}=0.036)$. Obese patitents (BMI $>30$ ) had significantly higher rate of complications in the peri- and post-operative course $(9.2 \%$ vs. $1.3 \%)$ and longer hospitalization time $(4.8 \pm 1.1$ vs. $2.9 \pm 1.4$ days; $\mathrm{p}=0.42$ ) than non-obese patients. Conclusion Reduced levels of adiponectin and elevated levels of resistin and proinflammatory cytokines (IL-1? and TNF-a) may be used as markers of complexity of perianal fistulas. Also those patients have a higher rate of complication during the peri- and post-operative course. Obesity has strong impact on the adipokines and cytokines serum levels and may be linked to poor outcomes of fistula treatment.

\section{ASSESSING THE QUALITY OF LIFE IN PATIENTS WITH INFLAMMATORY BOWEL DISEASE UNDERGOING SURGERY}

\section{Anuradha Ashok Thrikandiyur, Rajat Raghunath, Sharmila Gupta}

ESNEFT Colchester, Essex, GB

Background/Aim To assess the effect of surgery on the quality of life of patient with inflammatory bowel disease. Methods Patients undergoing surgery for Inflammatory Bowel Disease in a single centre between 2015 and 2019 were administered the SF-36 quality of life questionnaire preoperatively and 6 weeks, 6 months and 1-year postoperatively. The mean and standard deviation of the scores were calculated for each dimension, across the 4 different time frames. In each domain the scores were compared with its immediately succeeding score. Multiple paired T-tests were performed in this manner. Results This study had 90 participants with 42 (46.7\%) women. The operative interventions included both small and large bowel resections. Eight dimensions of the SF-36 were studied. General health: Mean score was 37.07 preoperatively, 57.58 at 6-weeks post-op, 61.02 at 6-months post-op and 57.4 at 1-year post-op. Physical functioning scale: Mean score was 66.59 preoperatively, 70.81 at 6-weeks post-op, 82.26 at 6-months post-op and 80.26 at 1-year postop. Pain scale: Mean score was 42.91 preoperatively, 63.08 at 6-weeks post-op score,72.27 at 6-months post-op and was 76.28 at 1 -year post-op. Social functioning scale: Mean score was 59.03 preoperatively, 67.26 at 6 -weeks post-op, 75 at 6-months post-op and 77.24 at 1-year post-op. Emotional well-being scale: Mean score was 60.43 pre-operatively, 72.62 at 6-weeks post-op, 70.22 6-months postop and 68.82 at 1-year post-op. Energy and Fatigue scale: Mean score was 29.43 preoperatively, 44.75 at 6-weeks post-op, 53.02 at 6 -months post-op and 51.6 at 1 -year post-op. Role limitation due to the emotional health scale: Mean score was 55.22 preoperatively, 60.84 at 6-weeks post-op, 79.54 at 6-months post-op and 80.34 at 1-year post-op. Role limitation due to physical health scale: mean score was 32.72 preoperatively, 29.58 at 6-weeks post-op, 72.77 at 6 -months post-op and 73.68 at 1 -year post-op. Conclusion There is a significant improvement in the quality of life across all dimensions after surgery for inflammatory bowel disease. This may be an 
indication to consider early operative intervention in the decision making process for patients with inflammatory bowel disease.

\section{IMPACT OF ADHERENCE TO ERAS GUIDELINES ON OUTCOMES AFTER COLORECTAL RESECTION IN A MULTICENTER SETTING}

Ellen Coeckelberghs, Kris Vanhaecht, Deborah Seys, Bianca Cox, Gabriele Bislenghi, Albert Wolthuis, Andre d'Hoore

\section{$K U$ Leuven, Leuven, $B E$}

Background/Aim Enhanced Recovery After Surgery (ERAS) guidelines provide a framework to improve postoperative outcomes of patients after colon surgery. Despite growing evidence of effectiveness, adherence to these guidelines remains challenging. The goal of the present study was to investigate the association between adherence to ERAS guidelines and outcomes after colorectal resection. Methods This is an observational, non-commercial, multicenter retrospective pretest posttest study, performed in 13 hospitals in Belgium. Patient records of consecutive patients, admitted for nonmetastatic colon cancer surgery and above 18 years old, were analyzed. The first audit took place from June until November 2017, and the second from March until October 2019. Outcomes of interest were postoperative complications, LOS, readmission, and adherence to set of 12 ERAS interventions. Results A total of 566 patients were included. The mean overall adherence to ERAS guidelines increased from $41.6 \%$ in 2017 to $55.0 \%$ in 2019 . Nevertheless, patient outcomes were not significantly different between periods. Across both periods however, on patient level, length of stay (LOS) and postoperative complications were significantly reduced with increasing ERAS protocol adherence. Mean LOS was significantly shorter in patients with high protocol adherence (6-7 interventions: -1.95 days; $8-9$ interventions: -3.32 days; $10-12$ interventions: -3.99 days) compared with patients with low protocol adherence ( $<6$ interventions). The odds ratios (ORs) for postoperative complications were 0.65 (95\% CI, 0.33-1.28), 0.12 (95\% CI, 0.03-0.38), and 0.12 (95\% CI, $0.23-0.59)$ with $6-7,8-9$ and 10-12 ERAS interventions, respectively. Conclusion Improved adherence to the standardized ERAS guidelines is significantly associated with a decrease in length of stay and risk of postoperative complications, without increasing readmission rate. This relationship shows an incremental improvement, with increasing adherence. Therefore, it is important to implement an ERAS bundle and not focus on a limited number of interventions.

\section{COLONIC ADENOCARCINOMA FOLLOWING TOTAL COLECTOMY AND ABDOMINO- PERINEAL RESECTION FOR ULCERATIVE COLITIS: CASE REPORT AND REVIEW OF LITERATURE}

\section{Krishanth Naidu, Travis Ackermann, Ian Davis, Hazel Serrao-Brown Garran \\ The Canberra Hospital, Canberra, Australia}

Background/Aim IBD-associated colorectal cancers usually arise from dysplastic mucosa, and can still develop after colectomy in patients with a rectal stump, ileorectal anastomosis and ileal-pouch anal anastamosis, with an incidence of $1-3 \%$. However, to the best of our knowledge there have been no published reports of colorectal adenocarcinomas arising in patient post total colectomy and abdomen-perineal resection for ulcerative colitis. Methods An otherwise healthy 77-year-old man was admitted to our hospital in acute renal failure with severe left-sided hydronephrosis, secondary to extrinsic compression by a large pelvic cyst. He reported symptoms of anorexia and lethargy on a background of deteriorating renal function. His past medical history is significant for ulcerative colitis for which he required a total colectomy, abdominoperineal resection and end ileostomy in 1968 for colitis refractory to medical management. To the patient's best knowledge, the resection specimen harboured no malignancy. Furthermore, a consultant Radiologist confirmed the anal canal to be absent. Results CT-urogram demonstrated marked left sided hydronephrosis and a complex cystic lesion $(12 \times 10 \times 14 \mathrm{~cm})$. The cyst was of homogenous density and was located anterior to the sacrum. His renal obstruction was managed with insertion of a left retrograde ureteric stent. Surgical drainage of the cyst was undertaken and malignancy was confirmed. Both cytology and histopathology showed moderately differentiated adenocarcinoma, composed of cribriform glands, lined by tall columnar cells with basal located nuclei and glands showed central comedo necrosis. On immunohistochemistry they were positive for CK7, CK20, CXD2 and SATB2. The Ki-67 proliferative index was very high. Overall, the morphology and immunoprofile are of adenocarcinoma of colorectal origin. Subsequent CT PET staging revealed no metastatic disease. The patient declined further surgical opinion from a specialist pelvic exenteration unit. Conclusion To our knowledge, this is the first case reporting colorectal adenocarcinoma following a total colectomy and abdomino-perineal resection for IBD. Endoscopic surveillance in this unique case is therefore impractical and the diagnosis challenging. Therefore, a high degree of suspicion is required in patients who present with indeterminate pelvic nodules or cysts in patients with a history of long-standing IBD, even in the absence of rectal or anal mucosa.

\section{ILEOSTOMY FORMATION IN COLORECTAL SURGERY INCREASES THE RISK OF ACUTE KIDNEY INJURY ACCORDING TO THE MODIFIED KDIGO CRITERIA}

\section{Krishanth Naidu Garran, Xiaotong Cheryl Tan, David Sheridan Rangiah}

The Canberra Hospital, Canberra, AU

Background/Aim Loop ileostomy construction is commonly used to attenuate septic complications associated with anastomotic leakage in low anterior resections (LAR). Post-operative dehydration amongst others, has deleterious consequences to electrolytes and renal profile culminating in acute renal failure (ARF). The aims of this study is to examine the epidemiology of loop ileostomy construction and its consequential local and systemic insults in those with left sided colonic resections. Secondarily, we intend to analyse the difference between early and late ileostomy reversals. We also aim to identify risk factors for ARF that may influence a patient's survival and quality of life. Methods A retrospective case-control review was conducted on colorectal cancer patients who underwent surgery over a 5-year period from 2011 to 2016 at 2 tertiary public hospitals in Australia. Records were accessed from the institutions' electronic medical records and discharge diagnosis codes to ensure all episodes were captured. The study group included those with a temporary ileostomy formed during a major left-sided colorectal cancer surgery. Patients who are under 18 years of age and did not have follow-up blood test for comparison were excluded. ARF was defined using the modified KDIGO criteria. Results Of 467 patients undergoing anterior resection surgery, $43 \%$ had ileostomies constructed. The median 
age of patients was 66 years with a male preponderance. Reassuringly, all patients with ileostomies had enteral continuity restored at a median time of 6 months $(n=200)$. Pre-existing renal failure rates was approximately $4.5 \%$ in both study groups. There was no septic or terminal complications in both cohorts. In the early post-operative days, there was inconsequential differences in the ARF rates between the cases and controls. When those with loop ileostomies are analysed, the risk of developing ARF is 2.6 times that of the control group $(13.5 \%$ vs $5.6 \%$; OR $=2.62 ; p=0.012)$. Of note, in those with an ileostomy, there is approximately 19- and 2.3-times risk of ARF when burdened with diabetes and anti-hypertensives, respectively. Conclusion We confirmed that after major left-sided colorectal resection surgery, there was a significant risk of developing ARF with an ileostomy (13\%). Though morbid, the mortality risk with stoma formation is null. In this study, pre-existing diabetes and antihypertensives increased the risk of ARF when an ileostomy is present.

\section{RANIBIZUMAB INDUCED ISCHAEMIC COLITIS: A REPORT AND REVIEW OF LITERATURE}

\section{Naren Kumaran}

\section{Kettering General Hospital, Leicester, GB}

Background/Aim Intravitreal injections of vascular endothelial growth factor (VEGF) inhibitors for exudative age-related macular degeneration (AMD), diabetic retinopathy, neovascular glaucoma and retinal vein occlusions are becoming the most frequent procedures in ophthalmology. In the randomized Comparison of Age-Related Macular Degeneration Treatments Trials (CATT) that assessed the relative efficacy and safety of ranibizumab (Lucentis, Novartis Pharma, Basel, Switzerland, and Genentech, South San Francisco, Calif., USA) and bevacizumab (Avastin, Genentech) for exudative AMD treatment, the incidence rates of serious systemic adverse events were higher in the bevacizumab-treated patients than in the ranibizumabtreated patients; in particular, the occurrence of gastrointestinal disorders was significantly higher with bevacizumab (3.0\%) than with ranibizumab (1.0\%).For this reason Ranibizumab is preferred.However it is more expensive than bevacizumab.We present a case of Ischaemic Colitis after an intravitreal injection of Ranibizumab.This has never been reported in literature. Methods We present a case of a 62-year-old woman who presented with multiple episodes of diarrhoea mixed with blood and severe abdominal pain after an intravitreal injection of Ranibizumab.The patient had an elevated white cell count of about 18 and a high lactate of 4 . A CT abdomen and pelvis was done which showed mucosal edema and thumb printing in the distal transverse colon, descending and proximal sigmoid colon. These signs were consistent with a diagnosis of ischaemic colitis. Results The patient was managed conservatively with intravenous fluids and nutrition. The patient was eventualy discharged home. A follow up CT Angiogram done 3 months after the initial episode showed complete resolution. Conclusion Irrespective of the safety profile, several monoclonal antibodies can cause a variety of gastro intestinal complications. The surgeon should specifically ask for a thorough medication history in patients presenting with ischaemic colitis. The adverse event should be reported and further doses must be avoided. Despite being expensive Ranibizumab is preferred because it is thought to have lesser side effects.Is this extra cost justified given the fact that even Ranibizumab is not immune to adverse effects.

\section{LOOP ILEOSTOMIES IN RECTAL CANCER}

\author{
Filipa Campos Costa, Inês Alegre
}

\section{Lisbon, $P T$}

Background/Aim In colorectal cancer a diverting stoma is used to reduce the clinical consequences of low colorectal and colo-anal anastomosis leaks. The decision to use a defunctioning loop ileostomy to protect an anastomosis is based on whether there are factors that affect the healing of the anatomosis, as well on the assessment of the individual surgeons. When creating a defunctioning loop ileostomy, the aim is to reverse it within $8-12$ weeks. The aim of this study was to determine time until ileostomy closure in colorectal cancer patients who had their ileostomy done to protect an anastomosis. We also tried to identify complications related to loop ileostomies and risk factors for nonclosure. Methods All consecutive patients between January 1, 2016 and December 31, 2019 who had a colorectal/coloanal anastomosis done for rectal cancer were identified. Clinical data from the patients who had a defunctioning ileostomy done to protect an anastomosis were retrospectively retrieved from patient records. Data were collected in July 2020. Results During the period of evaluation 115 patients were submitted to a rectal cancer surgery with or without an ileostomy, excluding transanal excisions. Thirty-three percent (38/ 115 ) had a diverting loop ileostomy due to a preoperative or intraoperative decision with the purpose of protecting the anastomosis. Thirty-seven percent of patients (14/38) had one or more complications related to their loop ileostomy: parastomal skin ulceration $(6 / 14)$ and high-volume output (4/14) were the most common. Eighty-two percent of patients $(31 / 38)$ had their stoma reversed within the period of evaluation. Median time until closure was 259 days. Delay in ileostomy closure was mainly due to adjuvante chemotherapy, preclosure investigations and lack of theatre time. Seven patients (7/38) did not have their stoma reversed within the period of evalution. When evaluating the risk factos for nonclosure the main risk factor was progression of the disease (6/7). Conclusion The time until closure was much longer than the suggested. The morbidity related to loop ileostomies is also considerable as well as the number of patient that do not have their ileostomy closed. Patients should be informed of these issues prior to the índex surgery.

\section{IMPACT OF SMOKING ON OUTCOMES POST RIGHT HEMICOLECTOMIES}

\section{Sarit Badiani, David Goltsman, Chris Berney}

Bankstown Hospital, Sydney, AU

Background/Aim Research has shown that compared to non-smokers, smokers have a higher chance of surgery-related complications. There is no data on the specific impact of smoking following right hemicolectomies $(\mathrm{RH})$ for cancer resections. The aim of this study was to determine the effect of smoking on post-surgical outcome following RH. Methods Data was obtained from the ACS-NSQIP database between 2014 and 2018. Logistic regression models were used to examine the association between smokers and non-smokers, with regards to medical or surgical complications. The statistic of interest in these analyses was the odds ratios $[95 \%$ (CI)] and their p-values of smokers and non-smokers. Subgroup analyses were performed based on method of resection: open, minimally invasive (laparoscopic/SILS) and robotic. RH commenced laparoscopically or robotically, but converted to open were overall classified as open procedures. Results A total of 18,889 RH patients were identified out of which, 10,861 (57.5\%) underwent a laparoscopic approach, 6657 $(35.2 \%)$ an open procedure and 1381 (7.3\%) a robotic 
resection. There were $2313(12.2 \%)$ smokers in this population. Twenty-four percent $(4475 / 18,889)$ of patients experienced postoperative complications. Logistic regression analyses showed that smokers experience a significantly higher rate of medical complications (OR: 1.43, p-value: <0.001), surgical complications (OR: 1.20, p-value: 0.002), wound complications (OR: 1.50 , p-value: $<0.001$ ), and anastomotic leaks (OR: 1.56, p-value: $<0.001$ ). On subgroup analysis smoking mostly affected outcomes in the minimally invasive approach increasing the odds of wound complications (OR: 1.54, p-value: 0.005) and anastomotic leaks (OR: 1.70, p-value: 0.003). Smoking affected the odds of medical complications in open procedures (OR: 1.34, p-value: 0.002) and surgical complications in robotic procedures (OR: 1.74, p-value: 0.027). Conclusion This outcomebased study demonstrates that patients diagnosed with right-sided colon cancer who are smokers have a significantly increased relative risk of developing major post-operative complications, compared to non-smokers. There is a strong argument for the cessation of smoking prior to embarking on surgery even in the elective setting.

\section{LAPAROSCOPIC POSTERIOR PELVIC ORGAN PROLAPSE SUSPENSION (PPOPS) TECHNIQUE WITH A BRAND NEW PRE-FOLDED POLIURETHANE COATED MESH: AN INNOVATIVE APPROACH FOR COMPLEX PELVIC ORGAN PROLAPSE}

Giacomo Carganico, Francesco Calabrese, Giorgio Querini, Andrea Caneparo, Marco Giacometti, Renza Trapani, Federico Lovisetto, Sandro Zonta

\section{Ospedale Unico Plurisede ASL VCO, Verbania, IT}

Background/Aim Descending perineum syndrome due to pelvic prolapse is defined as the descent of the pelvic organs due to weakness of the supporting structures of the pelvic floor. When pelvic prolapse mainly affects the organs of the posterior compartment, proctological dysfunctional symptoms become prevalent in the clinical picture. Here we describe an innovative technique called pPOPS: this is inspired by the POPS technique used in gynecology but it is specific for the middle and posterior pelvic compartment. Methods We treated 25 female patients with pelvic prolapse from 2018 to 2020 with the pPOPS technique. After inducing pneumoperitoneum with a Veress needle introduced in the left subcostal, three trocars are placed and the uterus is suspended. The pre folded Polyurethane-coated mesh (Wing Mesh Pro Compact ${ }^{\circledR}$, Angiologica s.r.l.-Italy) grants a quick and easy positioning, avoiding time waste for plain developing of the mesh in the pelvis. The Polyurethane-coated mesh is fixed to the posterior fornix and to the anterior wall of the rectum with $2 / 0$ polypropylene detached sutures. Only Polyurethane layer touches the hollow viscera. The wings of the mesh are then extended bilaterally through a pre-peritoneal tunnel created through an incision made at the level of the antero-superior iliac spine and fixed to the fascia of the external oblique muscle under traction to ensure the correct suspension of the pelvic organs. Results All patients treated were discharged in the 5 th post-operative day. Mean operative time was $42 \pm 13 \mathrm{~min}$. No intraoperative complication was reported, all patients reported an improvement in symptoms. No complication related to mesh implant such as erosion, infection was highlighted along with patients follow up. Radiological post-operative examination documented the markedly reduction of the perineum descendent syndrome in all patient treated. No recurrency was reported. Conclusion The use of the pre-folded Polyurethane mesh showed significant reduction of the operative time, ease of positioning in the operative field that meant improved effectiveness in pelvic prolapse correction. Then, the use of polyurethane layer increases the patient's safety regarding the risk of infection and erosion.

\section{IMPACT OF NEOADJUVANT CHEMOTHERAPY INTERVAL ON TUMOUR REGRESSION GRADING FOR RECTAL CANCER}

Thomas Tiang, Ankur Sidhu, David Williams, Andrew Bui

Austin Health, Heidelberg, AU

Background/Aim Colorectal Cancer is the third most common in Australia with almost 17,000 new diagnosis per year. The time interval of surgery after completion of neoadjuvant chemoradiotherapy (nCRT) has been a point of debate in rectal cancer management. This study investigates the tumour regression grade in rectal cancer patients, primarily if interval of surgery after nCRT has an impact on tumour regression grade and disease recurrence. Methods Data was collected from from 2009 to 2015. Primary outcomes being tumour regression grade of the rectal specimen. Logistic regression modeling is utilized to determine if interval of surgery has an impact on tumour regression grade, adjusting for other factors such as age, sex, operation, location of tumour and pre and post-operative $\mathrm{T}$ and $\mathrm{N}$ stage. Results There were 94 patients that satisfied the inclusion criteria of neoadjuvant chemoradiotherapy and progressed on to Total mesorectal excision, either with or without anastomosis. $60 \%$ patients had an anastomosis, $16 \%$ had excision without anastomosis, either APR or Hartmanns, $3 \%$ had a local resection. Of the 56 patients that had an anastomosis $70 \%$ had this procedure done laparoscopically. Median Age of this cohort was 62. The gender proportion was M:F [36\%:73\%]. Conclusion Tumour regression grade was not reported for all patients, 52 patients in total had a valid grading performed. There was a complete pathological response rate of $13.4 \%$. Performing a multivariate logistic regression analysis adjusting for Age, Gender, Location and Operation. Interval to surgery does not show a statistically significant effect on Tumour regression grading. The $\mathrm{p}$-value for this factor in the model is 0.478 indicating interval to surgery does not have a significant impact on TRG.

\section{CARDIOPULMONARY EXERCISE TESTING (CPET) IN HIGH RISK COLORECTAL SURGERY PATIENTS SHOULD BE USED TO MODIFY PERIOPERATIVE PATHWAYS IN ORDER TO OPTIMISE OUTCOMES}

\author{
David Berry, Rosie McDonald, Andrew Packham, Andrew Miller
}

Leichester Royal Infirmary, Leichester, GB

Background/Aim Cardiopulmonary exercise testing (CPET) provides an objective measure of a patient's cardio-respiratory functional capacity under stress. Previous studies have suggested that CPET parameters can predict postoperative morbidity and mortality. The aim of this study was to explore whether CPET parameters of highrisk colorectal patients were predictive of outcomes. Methods Colorectal patients considered high risk for surgery and referred for CPET over a one-year period were included. Demographics, CPET parameters (AT, Peak V02, VeqC02), operation details, complications, mortality and length of hospital stay were gathered from the patient's electronic records. Results Of the 99 colorectal patients referred, 85 completed CPET assessment and 59 proceeded to also 
have elective surgery. Of those proceeding to surgery mean age was 72.9 years, $52.5 \%$ were male, average BMI was 28.6 , mean AT was $8.8 \mathrm{ml} / \mathrm{kg} / \mathrm{min}$, mean Peak V02 was $14.0 \mathrm{ml} / \mathrm{kg} / \mathrm{min}$ and mean VeqC02 was $40.6 \mathrm{~L} / \mathrm{min}$. Of those not proceeding to surgery mean age was 69.4 years, $61.5 \%$ were male, average BMI was 30.5 , mean AT was $7.5 \mathrm{ml} / \mathrm{kg} / \mathrm{min}$, mean Peak V02 was $11.6 \mathrm{ml} / \mathrm{kg} / \mathrm{min}$ and mean VeqC02 was $43.5 \mathrm{~L} / \mathrm{min}$. Sixty-five discrete complications were tabulated against AT, Peak VO2 and VeqC02 with all 8 Clavien Dindo IIIa or higher complications being in patients with an AT $<10$. Of the patients with an AT $\geq 1041.9 \%$ had an uncomplicated admission. Mean length of stay was 13.2 days. Return to theatre rate was $6.5 \%, 6.5 \%$ were discharged to a rehabilitation placement and readmission rate was $11.3 \%$. There was 1 mortality in the postoperative period $(1.6 \%)$. Conclusion This study identifies that whilst there may be an association between CPET parameters and major complications (Clavien Dindo III-V) a significant proportion of patients considered high risk have an uncomplicated hospital admission. This may reflect CPET being used to risk stratify patients and outcomes being optimised by careful perioperative planning.

\section{LHP FOR THE TREATMENT OF HEMORRHOIDAL DISEASE, EXPERIENCE OF 350 CASES}

\section{Ionel H. Roman \\ Clinique CIC Valais, LA CONVERSION, CH}

Background/Aim Background Laser hemorrhoidal procedure (LHP) is a new minimally invasive technique to treat symptomatic hemorrhoids. The aim of this study was to draw some preliminary conclusions regarding the treatment of hemorrhoidal disease by laser, using the LHP technique, after performing more than 350 interventions of this type. Methods Indications for LHP included patients with symptomatic hemorrhoids resistant to medical therapy, with lowmedium grade of prolapse. Clinical efficiency was evaluated assessing resolution of symptoms and patient satisfaction. We compared the acceptability, postoperative evolution and levels of pain, hospitalization, and overall short time results after more than three years of experience with LHP. It is important to mention that in our practice we apply a large palette of interventions for the treatment of hemorrhoidal disease, including Doppler, Fergusson or even more classical techniques. Results The good short term results, confirmed also by the studies already published, rise the question if the LHP would not be a major breakthrough in the treatment of hemorrhoidal disease. Conclusion The LHP procedure seems to be safe and effective in patients with symptomatic hemorrhoids. It is simple, minimally invasive, and relatively pain free. It can be performed in an ambulatory setting, and it achieves high patient satisfaction.

\section{THE SURGEON PSYCHE: A PRELIMINARY ANALYSIS OF COLORECTAL SURGEON PERSONALITY}

\section{Carly Bisset, Nicola Dames, Susan Moug \\ The Plato Project Steering Group On behalf of Royal Alexandra Hospital, Paisley, GB}

Background/Aim The Edinburgh Delphi (2016) was the first study to investigate the potential role of personality on anastomotic decision making. The authors from that study found that surgeons displayed high levels of conscientiousness and emotional stability compared to population norms. A patient and public involvement study demonstrated that patients believe that post-operative outcomes may be influenced by surgeon personality. We aimed to perform a preliminary analysis of the first 50 participants undertaking The Plato Project survey. This may allow targeted non-technical skills training and gain insight into the surgeon's cognitive bias via a reflexivity intervention. Methods The Snap11 online survey tool was used to create a descriptive and quantitative study; collecting data on: surgeon demographics (age, gender, country of practice, experience), validated personality testing (44-item Big Five Inventory) and a validated risk-taking tool (30-item DOSPERT). Certified colorectal surgeons were invited to participate via social media and colorectal association correspondence. Results We present the preliminary results of the first 50 responders from the survey. Surgeons from 15 countries participated, with the majority based in England (46\%). 34\% of responders were female $(n=17)$, with half of responders $(48 \%)$ aged between 40 and 49 years. 5 personality domains were reported using the Big Five Inventory, with UK population norms provided for context: extraversion (2.81 vs 3.24); agreeableness (3.02 vs 3.74); conscientiousness (3.44 vs 3.65 ); emotional stability ( 3.25 vs 2.97 ) and openness (3.15 vs 3.67). Risk-taking was reported using the following domains: ethical (1.99); financial (2.3); health and safety (2.81); recreational (3.56) and social (4.82) with UK population norms for comparison. Conclusion This cohort of colorectal surgeons have displayed lower levels of extraversion (more reserved than outgoing), agreeableness (more analytical than emotive) and openness (more objective than creative) compared to previous groups; with higher levels of emotional stability (performing well under pressure). Further analysis is necessary to validate these findings and identify if geographical variations in personality exist between surgeon groups.

\section{COMPARISON OF COLORECTAL CANCER OUTCOMES BEFORE AND DURING THE COVID- 19 PANDEMIC}

\section{Triantafyllos Doulias}

Colchester Hospital, Colchester, UK GB

Background/Aim The COVID-19 pandemic has significantly impacted all aspects of surgical practice, including colorectal cancer. We aimed to identify changes in colorectal cancer management and outcomes resulting from the COVID-19 pandemic. All colorectal cancer patients treated with curative intent during the peak COVID19 period in our region (March-June 2020) were identified. A comparison of operative approach and outcomes was made with patients from the same 3-month period of 2019. Methods 39 patients had elective surgery during the COVID period compared with 76 patients in the equivalent pre-COVID period, demonstrating a $46.8 \%$ reduction in elective activity. Additionally, 3 patients awaiting elective surgery during COVID presented with acute obstruction and underwent emergency surgery, with 1 mortality. Results $43.6 \%$ (17) of patients had laparoscopic surgery during COVID compared to $81.6 \%$ (62) pre-COVID [p = 0.01, Chi-Square]. During COVID, 56.4\% (22) had primary anastomoses, $10.3 \%$ (4) had temporary stomas, and $33.3 \%$ (13) had permanent stomas. Pre-COVID, 68.4\% (52) had primary anastomoses, $17.1 \%$ (13) had temporary stomas, and $14.5 \%$ (11) had permanent stomas [ $p=0.55$, Chi Square]. Median [IQR] length of stay was 6[5-9] days during COVID and 5[4-8] pre-COVID [p $=0.007$, Mann-Whitney U]. 30-day mortality was $2.6 \%$ during COVID compared with $0 \%$ pre-COVID. Conclusion A change in operative practice during COVID-19 is clearly demonstrated. A significantly higher proportion of patients underwent open rather than laparoscopic surgery during the COVID-19 period, reflecting a 
change in national UK guidance due to concerns regarding aerosolisation risk. Patients were also more likely to have a permanent stoma rather than anastomosis. This change in practice not only had an impact on post-operative recovery, but will also likely have a significant impact on quality of life in these colorectal cancer patients.

\section{TOLERABLE GASTROINTESTINAL IMMUNE RELATED ADVERSE EFFECT (IRAE) \\ OF NATURAL KILLER (NK) CELLS THERAPY ON ADVANCED STAGE COLORECTAL CANCER: A PRELIMINARY STUDY}

Budhi Ida Bagus Karanganyar, Setyawati Ida Ayu, Mastini Ida Ayu Kade, Metria Ida Bagus

\section{Sebelas Maret University, Karanganyar, ID}

Background/Aim Adjuvant therapy for advanced stage colorectal cancer was so challenging, many guidelines already been updated for the better clinical outcome of these colorectal cancer patients. Chemotherapy alone has many variable outcome on the patients. On the last decades, immunotherapy has an important role on giving the better outcome as a combination with standard chemotherapy or as monotherapy. Especially in our developing country, funding issues was the common problems, we have lack of data about the effectivity or adverse effects of this promising therapy like natural killer cells. Latest study state that $44 \%$ cases related to gastrointestinal irAEs. Methods We will evaluated the gastrointestinal tolerability and degree of immune related adverse effect which could be presented during natural killer cells therapy as monotherapy on stage III colorectal cancer. The patient was resectable colorectal cancer with no neo-adjuvant chemotherapy history before. Any gastrointestinal symptoms will be reported. Results We reported a case of 67-year-old female patients with transverse colon adeno carcinoma and lymph node involvements confirmed by histopatology results. R0 resection has been done, NK cells monotherapy has started and continued for 2 course with 2 months interval. During this evaluation, she has a mild diarrhea associated with colitis and can be managed conservatively without interruption on this immunotherapy cycle (grade I). The patient was tolerated well with the NK cells therapy. Conclusion It might be to early to make a recommendation in our clinical practice, from this study, we can conclude that gastrointestinal related to NK cells immunotherapy was tolerable and can be managed conservatively. Keywords: gastrointestinal symptoms, immune related adverse effect, advanced colorectal cancer.

\section{DOES HAVING ENGLISH AS A SECOND LANGUAGE AFFECT THE ADEQUACY OF BOWEL PREPARATION IN PATIENTS UNDERGOING COLONOSCOPY?}

\author{
Ankur Sidhu Kensington, Jessica Rahme, Sharon Lee, Haider \\ Abdulrasool, Mark Tacey, Basil D'Souza
}

\section{Northern Health, Kensington, $A U$}

Background/Aim Colonoscopy remains the gold standard for diagnosis of adenomatous polyps and colorectal cancer. The success of colonoscopy is highly dependent upon the adequacy of bowel preparation. A range of risk factors have been identified that contribute to poor bowel preparation. Currently there is little research to suggest that having English as a second language leads to poor bowel preparation. The aim of this study is to determine whether having English as a second language contributes to poor bowel preparation. The secondary aim was to determine other factors contributing towards a poor bowel preparation. Methods This study was conducted as a retrospective audit. All patients who underwent colonoscopy at the Northern Hospital over a 3-month period were identified. From this cohort, patients who listed language other than English as their primary language were identified. The quality of bowel preparation was assessed through the colonoscopy reports. Descriptive statistics were prepared and multivariable logistic regression analysis conducted to identify factors associated with poor bowel preparation. Results A total of 1418 patients were identified who underwent colonoscopy during the study period. $1161(81.9 \%)$ patients were from English speaking background (ESB) and 257 $(18.1 \%)$ patients were from non-English speaking background (NESB). Within the NESB cohort, an interpreter was utilised for $72.8 \%$ of patients for their clinic consultation and consent process. The incidence of poor bowel prep was $10.4 \%$ in ESB patients and $10.9 \%$ in NESB patients $(\mathrm{p}=0.36$ ), with no significant difference found via multivariable analysis (NESB vs ESB: OR 1.05, 95\% CI: $0.67-1.63, \mathrm{p}=0.83)$. The variables that were associated with poorer bowel preparation included male sex (OR 1.53, 95\% CI 1.09-2.16, $\mathrm{p}=0.015)$, extended bowel preparation (OR 2.40, 95\% CI 1.43-4.02, $\mathrm{p}=0.001$ ) when compared to Prep Kit C/Moviprep and having colonoscopy on the morning list compared to an afternoon list (OR $1.51,95 \%$ CI $1.07-2.15, \mathrm{p}=0.021)$. Conclusion There was no difference in bowel preparation between ESB and NESB patients undergoing colonoscopy. This could be related to high rate of interpreter utilisation. Other variables associated with poorer bowel preparation were similar to previous studies.

\section{THE CHANGING PHASES OF ACUTE APPENDICITIS MANAGEMENT DURING THE FIRST WAVE OF COVID-19 IN THE UNITED KINGDOM}

\section{Hannah Javanmard-Emamghissi Derby, Eleanor Massie}

University of Nottingham, Derby, GB

Background/Aim Acute appendicitis (AA) is the most common general surgical emergency worldwide. The World Society of Emergency Surgery (WSES) guidelines endorse conservative management (CM), with antibiotics, as one safe and effective treatment of AA, yet in the United Kingdom (UK) laparoscopic appendicectomy remains the standard of care. During the COVID-19 pandemic, established professional bodies supported changing surgical practice to $\mathrm{CM}$ to avoid the potential risk of virus aerosolization during laparoscopy, and increased mortality in patients infected with COVID-19 peri-operatively. Here we report on the response of the UK surgical community to these proposals. Methods This prospective, multi-centre cohort study included participants aged 18 years and older with clinically or radiologically diagnosed AA. Data was collected from 23rd March 2020 to 23rd June 2020. National guidelines were collated from the Surgical Colleges and the Association of Laparoscopic Surgeons of Great Britain and Ireland (ALSGBI). Shortly before the peak of the pandemic (Phase 1), intercollegiate guidelines were released recommending that non-surgical solutions be used where possible, with further clarification on March 27th that AA should be managed either conservatively or with open surgery. ALSGBI guidelines released on April 22nd (Phase two-decline) advised consideration of laparoscopic surgery where safety measures could be met. On May 30th (Phase three-recovery) the intercollegiate guidelines advised local risk assessment regarding laparoscopy. AA 
management in these phases was compared with the changing guidelines. Results 3107 participants with a clinical or radiological diagnosis of AA were included. During Phase 1, $48 \%$ of patients were managed conservatively, and only $18 \%$ laparoscopically. In Phase 2 $36 \%$ were managed conservatively, with $41 \%$ undergoing laparoscopic appendicectomy. By Phase $315 \%$ were managed conservatively and $76 \%$ with laparoscopic surgery. Conclusion During Phase 1, the majority of AA patients were treated conservatively, however by Phase 3, the majority had returned to the previous standard of laparoscopic appendicectomy. This work displays that general surgeons promptly adopted the rapidly changing guidelines, demonstrating the significant influence of surgical professional bodies on UK practice.

\section{THE ROLE OF TRANSCATHETER ARTERIAL EMBOLIZATION IN THE MANAGEMENT OF LOWER GASTROINTESTINAL BLEEDING: A META-ANALYSIS}

Krishna Kotecha, Ji Chen, Jothi Rajasekaran Murugesan, Timothy Shore, Lucian Roseverne Richard Maher, Alexander Engel

Royal North Shore Hospital, St Leonards, NSW, AU

Background/Aim The investigation and management of lower gastrointestinal bleeding (LGIB) is resource intensive. Following risk stratification, and investigation of source and location, management options include therapeutic endoscopy, transcatheter arterial embolization (TAE) and surgery. Despite widespread adoption of TAE in the management of LGIB, no randomised control trials comparing TAE with colonoscopy exists and is unlikely ever to be available. Meta analytic summary establishing summary thresholds for the rates of success, complications and rebleeding in TAE is the purpose of the present study. Methods We conducted a systematic review and meta-analysis of studies published between 1990 and 2019. A total of 50 studies were included in summative analysis via the PRISMA flowchart. A total of 1354 patients underwent TAE for LGIB were included. Results TAE showed an immediate technical success rate of $94.6 \%$. A total of 285 patients rebled at some point post TAE, of whom $89.8 \%$ rebled within 30 days, with an overall TAE rebleed rate of $16.1 \%$ (95\% CI $13.7 \%$ to $18.6 \%, p<0.0001)$. In 112 patients $(8 \%)$ intestinal ischaemia was reported, of which 41 (47\%) underwent surgical resection. 30 day all cause mortality rate following TAE was $9.7 \%$ and $2.9 \%$ for disease specific mortality. Conclusion This is the first meta-analysis that has shown that TAE is an effective treatment for LGIB with acceptable rebleed, complication and mortality rates.

\section{ROLE OF CIRCULATING MIRNAS AS BIOMARKERS DURING MULTIMODAL TREATMENT OF RECTAL CANCER-TIMISNAR- MIRNA: A SUBSTUDY OF THE TIMISNAR TRIAL (NCT03962088). PROTOCOL STUDY}

Igor Monsellato, Elisabetta Garibaldi, Elisa Cassinotti, Luigi Boni, Ugo Elmore, Roberto Delpini, Riccardo Rosati, Roberto Perinotti, Filippo Alongi, Elisa Bertocchi, Stefania Gori, Giacomo Ruffo, Graziano Pernazza, Raffaello Mancini, Angela Torsello, Fabio Pulighe, Carlo De Nisco, Emilio Morpurgo, Tania Contardo, Davide Cavaliere, Leonardo Solaini, Giorgio Ercolani, Valter Torri, Daniela Rega, Delrio Paolo, Surgo Alessia, Alba Fiorentino, Alessandro
Verbo, Massimiliano Ardu, Francesco Riente, Vittorio Fusco, Sara Orecchia

Antonio e Biagio e Cesare Arrigo Hospital, Alessandria, IT

Background/Aim Neoadjuvant chemoradiotherapy followed by surgery is the mainstay treatment for locally advanced rectal cancer, leading to significant decrease in tumor size (downsizing) and a shift towards earlier disease stage in the primary tumor and lymph nodes (downstaging). Extensive histopathological work-up of the tumor specimen after surgery including tumor regression grading and lymph node status helped to visualize individual tumor sensitivity to chemoradiotherapy, retrospectively. Since the response to neoadjuvant chemoradiotherapy is heterogeneous, however, valid biomarkers are needed to monitor tumor response. A relevant number of studies aimed to identify molecular markers retrieved from tumor tissue while the relevance of blood-based biomarkers is less stringent assessed. MicroRNAs are currently under investigation to serve as blood-based biomarkers. To date, no screening approach to identify relevant miRNAs as biomarkers in blood of patients with rectal cancer was undertaken. The aim of the study is to investigate the role of circulating miRNAs as biomarkers in those patient included in the TiMiSNAR Trial. This is a biomolecular substudy of TiMiSNAR Trial (NCT 03465982). Methods All included patients in the TiMiSNAR Trial are supposed to undergo blood collection at the time of diagnosis, after neoadjuvant treatment, after 1 month from surgery and after adjuvant chemotherapy whenever indicated. Results TiMiSNAR-MIRNA will evaluate the association of variation between preneoadjuvant and postneoadjuvant expression levels of miRNA with pathological complete response. Conclusion Moreover, the study will evaluate the role of liquid biopsies in the monitoring of treatment; correlate changes in expression levels of miRNA following complete surgical resection with disease-free survival and evaluate the relation between changes in miRNA during surveillance and tumor relapse.

\section{PNEUMOMEDIASTINUM AFTER HAEMORRHOIDOPEXY. A UNIQUE CASE?}

Francisco Girão de Caires Funchal, António Girão de Caires, Tiago Branco, Regina Silva, Rita Camarneiro, Isabel Dionisio, Adelaide Costa, António Martins

\section{Centro Hospitalar do Oeste, Funchal, PT}

Background/Aim Haemorrhoidal disease is the most frequent reason for proctology consultation, and may be present in up to $70 \%$ of the working population. Haemorrhoid artery ligation (HAL) was first described in 1995 by Morinaga and have been improved with fixation of prolapsed tissue with mucopexy or recto-anal repair (RAR). [2] Complications with this procedure have been reported and include persistent pain, haemorrhage, rectal perforation, complex fistulas, peritonitis, faecal incontinence and anal stricture. Methods 59-yearold, presented to the emergency department with the main complaint of precordial discomfort. The patient had been submitted to an elective HAL-RAR due to grade IV hemorrhagic haemorrhoids two days prior to this episode. She had no other significant past medical history. Physical examination: Subcutaneous emphisema in the cervical region. No abnormalities in the Electrocardiogram and analytically were found. The computed tomography (CT-Scan) revealed an extensive emphysema of the parapharyngeal, retropharyngeal and sublingual space in addition to a pneumomediastinum and pneumo-retroperitoneum originated in the rectum. The patient was admitted under analgesia, dietary restriction and imagiologic 
control. She kept clinically stable with algic improvement and regression of the emphysema. She was discharged by the 8th day. By the post-op consult ( 29 days after) the CT-scan only showed a few peri-renal bubbles. Results The major advantage of HAL over other forms of treatment is the reduced postoperative discomfort and bleeding. This case has highlighted a potentially life-threatening complication of an HAL-RAR. Although similar cases have been described in other procedures, this case represents a yet to be described complication. Conclusion We still believe the use of HAL is an effective, feasible and safe treatment modality for symptomatic haemorrhoids. It should, however, alert physicians that, as with all surgical procedures, it is not free of potentially serious complications.

\section{LIMBERG FLAP VERSUS KARYDAKIS FLAP FOR TREATING PILONIDAL SINUS DISEASE: A SYSTEMATIC REVIEW AND META-ANALYSIS}

\section{Paschalis Gavriilidis Birmingham, Emil Bota}

Imperial College London, Birmingham, GB

Background/Aim The Limberg flap reconstruction and the Karydakis flap reconstruction are the 2 most used off-midline closure techniques in pilonidal sinus surgery. The current evidence is inconclusive as to which is the optimal technique. The aim of this systematic review and meta-analysis was to compare differences in outcomes between these 2 flap-based techniques. Methods We identified studies by a systematic literature search of the Embase, MEDLINE (PubMed), Cochrane Library and Google Scholar databases and studies selected as per the Preferred Reporting Items for Systematic Reviews and Meta-Analyses (PRISMA) checklist. Only randomized controlled trials (RCTs) that compared the Limberg flap (standard or modified) and the Karydakis flap were included in this review. Results Operative time was shorter by $7 \mathrm{~min}$ in the Karydakis group than in the Limberg group (mean difference $7.00 \mathrm{~min}, 95 \%$ confidence interval [CI] 0.53 to 13.48). The seroma formation rate was significantly higher in the Karydakis cohort (odds ratio [OR] 0.36, 95\% CI 0.24 to 0.56 ); however, after excluding studies with a high risk of bias, the sensitivity analysis showed no significant differences in seroma formation rate between the 2 techniques (OR $0.76,95 \%$ CI 0.31 to 1.85 ). Other outcomes of interest showed no significant differences between the Limberg and Karydakis techniques. Conclusion There were no significant differences between the Limberg and Karydakis techniques. Future RCTs with strict adherence to CONSORT guidelines will further elucidate the efficacy of these surgical procedures.

\section{IMPACT OF NEOADJUVANT CHEMORADIOTHERAPY ON POST OPERATIVE OUTCOME IN PATIENTS WITH RECTAL CANCER}

\author{
Shirish Tewari, Sophie Bondje, Vivek Gupta, Nick Reay-Jones \\ Lister Hospital, East and North Herts Trust, Stevenage, GB
}

Background/Aim Neoadjuvant chemoradiotherapy not only helps in downstaging the rectal cancer but also reduces the rate of local recurrence but it has its attendant risk factors. Our aim is to look into the implications of neoadjuvant chemo radiotherapy in patients with rectal cancer in our hospital. Methods Retrospective data was collected from the infoflex system of database for the patients who underwent neoadjuvant chemoradiotherapy between Jan 2016-Dec 2019 in our DGH. The data was analysed for demographics, type of surgery, histology, length of stay, complications and stoma reversal rates. Results 27 Patients with rectal cancer during the study period underwent neoadjuvant chemoradiotherapy. 16 were male and 11 females. Age range was 33-82 year. 13 patients underwent APER, 8 Hartmann's and 6 anterior resection with defunctioning loop ileostomy. 5 patients initially required defunctioning sigmoid loop colostomy and one loop ileostomy prior to start of neoadjuvant treatment. Histology showed 26 adenocarcinoma and 1 had squamous cell cancer. 3 patients had complete pathological response. Post operative stay ranged from 5 to 32 days. 12 patients developed pelvic collection, 5 wound infection( 4 perineal +1 abdominal), 5 had paralytic ileus, 1 patient had PR bleed, 2 anastomotic leak and 2 patients had chest related complications. Only 4 out of 6 loop ileostomies were reversed. Conclusion Neoadjuvant treatment benefits comes with its attendant complications. The tissue response to radiotherapy and chemotherapy and advanced tumor stage may contribute for the increased morbidity. Hopefully in future with the advent of new armamentarium the morbidity may come down but till then we have wait and watch.

\section{STUMPED BY APPENDDICITIS}

Francisco Girão de Caires, António Girão de Caires, Duarte Gil Alves, Carolina Silva, Rita Camarneiro, Ana Luiśs Rodrigues, Isabel Dionisio

\section{Centro Hospitalar do Oeste, Funchal, PT}

Background/Aim Appendicitis is one of the most common surgical emergencies in the world with an annual incidence of 10 cases per 100,000 population and laparoscopic appendectomy is the gold-standard regarding its management. Recurrent appendicitis is a rare entity, being reported approximately 1 in 50,000 appendectomies. It is typically caused by inflammation of the appendiceal stump. Appendicular stump closure is a critical step in this procedure, and several methods have been described to secure the appendicular stump during the laparoscopic appendectomy. Using Hem-o-lok clips to secure the base of the appendix is a safe and efficient option and, because of the price of the plastic clip and the simplicity of its application, its use is still highly favored during the laparoscopic procedure. Despite the afore mentioned, it has been proved that mild and moderate inflammation of the stump is more frequent when using Hem-o-lok clips than conventional stapler and cases of reaction to a foreign body have been reported when using Hem-olok clips. Methods We present a case of a 70-year-old male who had been submitted to laparoscopic appendectomy 2 years earlier subsequently presenting twice with a main complaint of right lower quadrant abdominal pain. On both episodes the definite diagnosis of stump appendicitis (SA) was established with a computed tomography and managed with antibiotic therapy. Due to his history the patient underwent successful elective laparoscopic removal of the appendiceal stump. Macroscopic examination of the appendiceal stump revealed 2 Hemo-o-lok clips in the lumen. The histopathologic analysis confirmed a chronic inflammatory reaction with cicatricial tissue. Results Although we cannot safely assert the etiology of this appendix inflammation, we cannot rule out the possibility that this might have been due to a tissue reaction to the Hem-o-lok clips. SA poses a diagnostic dilemma because of its infrequent nature and, if misdiagnosed, can lead to severe complications. Conclusion Despite this case we still believe that the use of Hem-o-lok clips is still a great option to secure the base of the appendix. 


\section{COMPLICATED METASTATIC MELANOMA TO THE GASTROINTESTINAL TRACT}

Rita Pereira Barreiro, Sara Patrocínio, Sofia Reis, Isa Santos, Zara Caetano

Centro Hospitalar Barreiro Montijo, Barreiro, PT

Background/Aim The annual incidence of malignant melanoma in the mediterranean countries is $7 / 100,000$ inhabitants, with a maximum peak at 65 -years. Ultraviolet radiation is the main carcinogenic agent. Most cases are diagnosed at an early stage when surgery is curative, however some patients have advanced disease at the time of diagnosis or develop metastases after initial treatment. Metastasis accounts for most of the morbidity and mortality. Methods Previously healthy 65 -year-old man diagnosed with malignant nodular melanoma on the back, was submitted to excision of the lesion and negative sentinel lymph node biopsy. Five years after the initial diagnosis, he developed nausea, anorexia and anemia. Upper digestive endoscopy identified a lesion in the duodenum whose biopsy was compatible with melanoma metastasis (M1c). Thoraco-abdominalpelvic CT scan revealed unresectable stage IV malignant melanoma. The patient started palliative chemotherapy and 2 months later he was admitted due to intestinal subocclusion, treated with endoscopic placement of a duodenal prosthesis. Due to the progression of the disease, he was re-admitted 1 month later with intestinal obstruction and an urgent gastrojejunostomy was performed. He was hospitalized 3 months later with focal neurological symptoms due to brain metastasis (M1d) and died 17 days later. Results Metastatic disease occurs approximately in $8 \%$ of patients two years after the initial diagnosis, with skin, lung and brain being the sites of choice. It is now known that gastrointestinal metastasis is not uncommon, occurring more frequently in liver and small intestine. However, less than $4 \%$ of patients with gastrointestinal metastases will develop symptoms during their lifetime, with the remaining cases being diagnosed only on autopsy. Gastrointestinal metastases portend a poor prognosis, with survival averaging 4-6 months. In the case of oligometastatic melanoma disease surgical resection should be performed to disease control. If curative resection is not possible, a palliative resection of intestinal metastases can be an appropriate treatment option for symptomatic disease, including intussusception, hemorrhage, perforation and obstruction. Conclusion Metastatic melanoma to the gastrointestinal tract poses a unique clinical challenge. In patients with previous history of cutaneous melanoma and complaints of abdominal pain or anemia, a study of the small intestine for an early diagnosis of metastases is recommended.

\section{RISK OF EMERGENCY SURGERY OR DEATH AFTER INITIAL NONOPERATIVE MANAGEMENT OF COMPLICATED DIVERTICULITIS IN SCOTLAND AND SWITZERLAND}

\author{
Marco von Strauss und Torney Basel, Giusi Moffa, Maximilan Kaech, \\ Fabian Haak, Stefan Riss, Elisabeth Deutschmann, Heiner Bucher, \\ Christoph Kettelhack, Hugh Paterson \\ Clarunis, Universitätsspital Basel, $\mathrm{CH}$
}

Background/Aim National guidelines on interval resection for prevention of recurrence after complicated diverticulitis are inconsistent. Although US and German guidelines favor interval colonic resection to prevent a perceived high risk of recurrence, UK guidelines do not. To investigate patient management and outcomes after an index inpatient episode of nonoperatively managed complicated diverticulitis in Switzerland and Scotland and determine whether interval resection was associated with the rate of disease-specific emergency surgery or death in either country. Methods This secondary analysis of anonymized complete national inpatient data sets included all patients with an inpatient episode of successfully nonoperatively managed complicated diverticulitis in Switzerland and Scotland from January 1, 2005, to December 31, 2015. The 2 countries have contrasting health care systems: Switzerland is insurance funded, while Scotland is state funded. Statistical analysis was conducted from February 1, 2018, to October 17, 2019. The primary end point defined a priori before the analysis was adverse outcome, defined as any disease-specific emergency surgical intervention or inpatient death after the initial successful nonsurgical inpatient management of an episode of complicated diverticulitis, including complications from interval elective surgery. Results The study cohort comprised 13861 inpatients in Switzerland (6967 women) and 5129 inpatients in Scotland (2804 women) with an index episode of complicated acute diverticulitis managed nonoperatively. The primary end point was observed in 698 Swiss patients (5.0\%) and 255 Scottish patients $(5.0 \%)$ (odds ratio, 0.98; 95\%CI 0.81-1.19). Elective interval colonic resection was undertaken in 3280 Swiss patients (23.7\%; median follow-up, 53 months [interquartile range, 24-90 months]) and 231 Scottish patients (4.5\%; median follow-up, 57 months [interquartile range, 27-91 months]). Death after urgent readmission for recurrent diverticulitis occurred in 104 patients $(0.8 \%)$ in Switzerland and 65 patients $(1.3 \%)$ in Scotland. None of the investigated confounders had a significant association with the outcome apart from comorbidity. Conclusion This study found no difference in the rate of adverse outcome (emergency surgery and/or inpatient death) despite a fivefold difference in interval resection rates.

\section{LOCAL STAGING OF RECTAL CANCER: HOW ACCURATE IS IMAGING?}

Joana Lemos Garcia, Miguel Braga, Isadora Rosa, Ricardo Fonseca, José Venâncio, António Dias Pereira

Instituto Português de Oncologia de Lisboa Francisco Gentil, E. P. E., Lisbon, PT

Background/Aim Rectal cancer is one of the most common malignant neoplasms of the gastrointestinal tract. Local staging is usually made by magnetic resonance (MRI), sometimes complemented by other methods, with variable diagnostic accuracy reported.Our goal was to evaluate the accuracy of rectal cancer staging in patients submitted to surgery with no neoadjuvant therapy. Methods Retrospective study. Patients with rectal cancer first assessed between January/2017 and March/2020 and directly referred to surgery after staging were included. Pathologic staging was considered gold-standard. Results 45 patients were included: $55.6 \%$ men, mean age 68.98 years-old. Upper rectum $=51.1 \%$, $\mathrm{mid}=35.6 \%$, lower $=$ $13.3 \%$. Local staging was made by MRI $=88.9 \%$ (15.6\% plus endoscopic ultrasound [EUS]) or computed tomography $=11.1 \%$ (4.4\% plus EUS). These methods accurately staged 3/7 T1, 8/12 T2, $14 / 23 \mathrm{~T} 3,3 / 3 \mathrm{~T} 4$ and $25 / 31 \mathrm{~N} 0,5 / 10 \mathrm{~N} 1$ and 1/2 N2. Imaging had sensitivity $\quad(\mathrm{Se})=92.0 \%, \quad$ specificity $\quad(\mathrm{Sp})=52.9 \%, \quad$ accuracy $(\mathrm{AUC})=81.4 \%$ for $\mathrm{T}$ staging; with no association between diagnostic accuracy and tumor location $(\mathrm{p}=0.723)$, pathological staging $(p=0.383)$, staging method used $(p=0.117)$ or time until surgery $(\mathrm{p}=0.646)$. Nodal involvement assessment showed $\mathrm{Se}=93.5 \%$, $\mathrm{Sp}=16.7 \%$, accuracy $=71.1 \%(75.6 \%$ in distinguishing N0 vs. $\mathrm{N}+$ ), with a statistically significant association between accuracy and $\mathrm{N}$ stage $(\mathrm{p}=0.014)$ - MRI correctly classified $80.6 \%$ of N0 patients 
and $45.5 \%$ of N1/N2 patients; no association was found with tumor location $(\mathrm{p}=0.112)$, method $(\mathrm{p}=0.314)$, time until surgery $(\mathrm{p}=0.805)$ or $\mathrm{T}$ stage $(\mathrm{p}=0.397)$. Discrepant cases had therapeutic implications in 5 patients ( 2 understaged as $\mathrm{T} 1$ and 3 understaged as N0). Conclusion As described in the literature, MRI showed good accuracy for $\mathrm{T}$ staging, but was only fair in $\mathrm{N}$ staging.

\section{QUALITY OF LIFE FOLLOWING CURATIVE SURGERY FOR COLORECTAL CANCER IN A SRI LANKAN COHORT; NORTH COLOMBO EXPERIENCE}

\section{Supun Godahewa, Kaushika Gunasekare, Shashikala Gajasinghe, Sumudu Kumarage, Pramodh Chandrasinghe \\ University of Kelaniya, Ragama, LK}

Background/Aim Colorectal cancer (CRC) is the 4th commonest cancer in Sri Lanka. It is increasingly approached with a curative intent due to the advancements in the surgical and medical management. This has resulted in a large cohort of cancer survivors that needs to be managed. Quality of life (QOL) and patient reported outcomes in CRC survivors assist in identifying and managing unique issues in this population. This study aims to describe objectively measured quality of life after surgery for colorectal cancer in a South Asian population. Methods Patients who underwent surgery for CRC and are currently disease free were included. QOL was assessed using the translated and validated SF36 questionnaire (Sinhala) along with patient complaints. SF36 components were aggregated into physical component summery (PCS) and mental component summery (MCS). Patient reported symptoms were categorized in to organ function, life style, and psycho-sexual. Results A Total of 100 patients completed the survey (median age-61 years; range 25-86, female-56\%). Of the total $65 \%$ were rectal cancers while $35 \%$ were colon cancers. QOL assessment reported a PCS of 0.64 and a MCS of 0.67 (Max1.0). There was no difference in QOL between rectal and colon cancers (PCS: rectal -0.64 vs colon-0.63, P = 0.9; MCS: rectal0.65 vs colon -0.66$)$ or based on follow-up period $(<5$ years vs $>$ 5 years; $\mathrm{P}=0.3$ and 0.9 ). Organ function related problems were reported by $69 \%$ and increased frequency was the main complaint (27\%). Conclusion The QOL of a Sri Lankan cohort of CRC survivors is described. QOL did not differ between rectal and colon cancers. Neither PCS nor MCS had significantly improved over time following surgery.

\section{SURVIVAL FOLLOWING CURATIVE SURGERY FOR COLORECTAL CANCER (CRC) IN THE MIDDLE-AGED POPULATION: DATA FROM A SOUTH ASIAN COHORT}

Pathum Vishwajith, Pramodh Chandrasinghe, Kaushika Gunasekare, Shashikala Gajasinghe, Sumudu Kumarage

University of Kelaniya, Ragama, LK

Background/Aim Colorectal cancer (CRC) is the fourth commonest cancer in Sri Lanka. Disease burden and survival among the middleaged population (50-70 years) with CRC is less studied. With the aging global population, this cohort has a high economic and a social impact. This study aims to analyse the survival pattern and contributing factors in a middle-aged patient cohort with CRC. Methods CRC patients managed with a curative intent between 1997 and 2020 at a specialised tertiary care unit were analysed. Demographic data, tumour characteristics and survival of the patients aged between 50 and 70 years was analysed. Overall survival was compared with the younger $(<50$ years) and older $(>70$ years) populations with CRC using Kaplan-Meire curves. Individual variable analysis was performed to sought for significant association of survival with age, sex, tumour stage and tumour site. Multifactorial analysis was performed using Cox-proportional hazard model. Results A total of 411 patients between 50 and 70 years age group with CRC were analysed (mean 60.07 years; range $-50-70$ years, male $-47.45 \%$ ). Overall survival of the middle-aged population was significantly better (mean133 months, SE 6.84) compared to the elderly population (mean58 month, $\mathrm{SE}=7.74$ ) but worse compare to the younger population (mean-167 month, SE $=10.81$ ). Majority of the tumours $(60.5 \%)$ in the middle-aged group were locally advanced on presentation $(>\mathrm{T} 3)$. Tumor site and age at presentation were significant factors that influence survival in this age group. Conclusion Middle-aged patients have a better mean overall survival than their older counterparts but fare worse compared to the younger age group. Tumor stage and age were the only factor that significantly influenced survival.

\section{IMMUNE DYSFUNCTION AS MEASURED BY THE SYSTEMIC IMMUNE-INFLAMMATION INDEX IS ASSOCIATED WITH THE SUB-TYPE OF MINIMAL RESIDUAL DISEASE AND OUTCOME IN STAGE II COLON CANCER TREATED WITH SURGERY ALONE}

\section{Nigel P Murray Santiago, Ricardo Villalon, Shenda Orrego, Eghon Guzman}

\section{University Finis Terrae, Santiago, $C L$}

Background/Aim Within 5 years after curative surgery for stage II colon cancer $25 \%$ of patients will relapse due to minimal residual disease (MRD). Immune dysfunction has been associated with a worse prognosis. We hypothesize that markers of immune function such as the systemic inflammation index (SII) is associated with the sub-type of MRD defined by bone marrow micro-metastasis (mM) and circulating tumour cells (CTCs). A higher immune dysfunction being associated with a more aggressive MRD and worse prognosis. Methods Blood and bone marrow samples were taken to detect CTCs and $\mathrm{mM}$ using immunocytochemistry with anti-CEA one month after surgery. The SII, (absolute neutrophil count $\times$ absolute platelet/absolute lymphocyte was determined one month post-surgery. These were compared with the sub-types of MRD; Group I MRD; Group II $\mathrm{mM}$ positive and Group III CTC positive; cut-off values of SII of $>700$ and $>900$ were used. Follow-up was for up to 5 years or relapse and survival curves using Kaplan-Meier (KM) were calculated. Results 181 patients (99 women) participated, mean age 68 years, median follow up 4.04 years; Group I: N = 105, Group II: $\mathrm{N}=36$, Group III: $\mathrm{N}=40$. The SII significantly decreased postsurgery only in Group I patients. The frequency of SII $>700$ and $>900$ was significantly higher in Group III, between Groups I and II there was no significant difference. The SII was significantly associated with the number of CTCs detected; patients with 1-2 CTCs/blood sample had a significantly lower median SII compared with patients with more than 2 CTCs/blood sample 739 (IQR 634-1081) versus 1084 (IQR 947-2032) respectively ( $<<0.05$ ). 53 (29\%) patients relapsed, with no significant difference between the median SII comparing patients who relapsed with those who did not, 919 (IQR 530-1090) versus $618(380-910)(\mathrm{p}=0.72)$ respectively. The 5-year KM was $98 \%$ Group I, 68\% Group II and 7\% Group III. 


\section{Conclusions}

The results of the study suggest that the severity of immune dysfunction after curative resection as determined by the SII is associated with differing sub-types of MRD and a worse prognosis. A significantly higher median SII, higher frequency of patients with a SII more than 700 or 900 was associated with CTC positive MRD. A more severe immune dysfunction was associated with an aggressive CTC positive MRD sub-type and a higher number of CTCs. Immune dysfunction has an important role in the type of MRD and prognosis in stage II colon cancer.

\section{SINGLE INCISION LAPAROSCOPIC SURGERY (SILS): IMPACT OF CHANGE IN PRACTICE ON PATIENT SATISFACTION}

\section{Gabriele DI Benedetto, Mariana Matias, Hansen Liemangow}

\section{York Teaching Hospital NHS trust, York, GB}

Background/Aim The aim of this study was to measure the effect of the switch in practice from MPL to SILS on patient satisfaction. Methods This was a retrospective cohort study on a population of unselected consecutive patients operated on by a single surgeon, as planned or unplanned surgery, at a high volume UK teaching hospital, between 01-04-2019 and 29-02-2020. Every patient aged 18-year-old or above, admitted with a significant colorectal pathology requiring a major resection, were enrolled in this study. The primary outcome was patient satisfaction, secondary outcomes were operative time, complication rate (Clavien-Dindo $>3$ ) and oncologic outcomes. Patient satisfaction was assessed by a telephone interview carried out by an independent doctor, who had not taken part in the index operation, at least three months after discharge, using a ten-point Likert scale questionnaire. The questions were targeted at exploring the rapport between patient expectation before surgery and the final result, wound cosmesis and recommendation to friends and family. Results The data were collected and analysed with an intention to treat protocol, using the median for continuous and mode for categorical variables. Statistical significance was evaluated using a twotailed Mann-Whitney test, with a p-value $<0.5$. Results A total of 56 adults underwent a major colorectal resection carried out by a single surgeon in this interval period (30 MPL-26 SILS), with an overall MIS completion rate of $95 \%$ (3/56). The two cohorts were well matched, with no significant difference in terms of age, BMI and ASA score, although there was a higher proportion of unplanned operations done in the MPL group (43\% vs $8 \%$ ). There was no statistically significant difference in operative time, complication rate and oncologic outcomes. The responses to the questionnaire recorded a similar high satisfaction rate in both groups. Conclusion Although SILS requires more advanced skills than MLS, it did not involve longer operative times and achieved similar high-quality results. It is interesting to note that the MLS group had a low complication rate, despite a high proportion of unplanned cases. This study failed to show any significant difference in primary and secondary outcomes between the two cohorts. Probable explanations of this is a small sample size and possible recall bias, which could be overcome with a prospective and powered randomised trial.

\section{RELEVANCE OF ROUTINE STUDY IN HEMORRHOIDECTOMY SPECIMENS: LATE RECURRENCE OF RECTAL ADENOCARCINOMA IN AN HEMORRHOIDS SAMPLE AFTER LOW ANTERIOR RESECTION}

Mariela Milagros Delgado Morales, Rocio Santos Rancaño, Alberto García García, Paz González Benjumea, Montserrat Reyes Moreno, Juan Francisco Guadalajara Jurado

Infanta Elena Hospital, Huelva, ES

Background/Aim Nowadays, there is an important controversy regarding the need for selective or systematic study of hemorrhoidectomy specimens for their pathological study. Methods We present the case of a 70-year-old man diagnosed with stenosing carcinoma in the lower-middle rectum (T3N2). After discussing the case in a Multidisciplinary Committee, the patient received neoadjuvant radiotherapy with a good response and we performed a low anterior resection. The histopathological study of the surgical specimen reported an adenocarcinoma with partial response and sixteen isolated lymph nodes with only one affected (T3N1). He received postoperative radioteraphy. Results One year after the surgery, he was referred again to our department due to a symptomatic hemorrhoidal nodule. Its exeresis was carried out and the histopathological report revealed infiltration of a well-differentiated adenocarcinoma, which ulcerated the epidermis and was in contact with the resection borders. An abdominoperineal resection was decided to be done. Conclusion Although many patients affected by colorrectal carcinoma develop metastases, an hemorrhoidal implant is extremely unusual. Metastatic rectal adenocarcinoma implants are most often located in anastomotic sites, perianal fistulas, biopsy areas and in perianal areas with a previous traumatic intervention and mucosal damage. Only three cases of metastasis in an hemorrhoid without previous therapeutic intervention have been reported in the literature. Optimal surgical treatment is controversial (with or without associated radiotherapy) ranging from local resection to abdominoperineal amputation in order to perform a radical exeresis. Our case concludes that it is a fact that an hemorrhoidal nodule may constitute a clinical presentation of an unsuspected primary carcinoma or could be an unusual form of colorrectal metastasis.

\section{INTRAOPERATIVE USE OF INDOCYANINE GREEN ANGIOGRAPHY TO REDUCE THE INCIDENCE OF ANASTOMOTIC COMPLICATION AFTER ROBOTIC LEFT SIDED COLORECTAL RESECTION}

\section{Asfar Ahamed Nawabjan \\ Apollo Main Hospital, Chennai, IN}

Background/Aim Anastomotic leak (AL) is a life-threatening complication after left sided colorectal resection with reported mortality of $10-15 \%$. Morbidity after anastomotic complication is dramatically increased which leads to reoperation, radiological intervention and permanent stoma. Most important cause is poor vascular supply. Microvascular impairment often not detected intraoperatively under white light. Indocyanine green angiography (ICG A) is useful technology to detect microvascular alteration and potentially preventing anastomotic complication. Methods From January 2015 to July 2020, 194 consecutive patients who underwent left sided robotic colorectal resection were enrolled and grouped into ICG A group $(n=95)$ and 
control group ( $\mathrm{n}=99)$. In ICG A group, all colorectal anastomotic perfusion observed with ICG enhanced robotic fluorescence imaging system (Firefly). Colonic perfusion was observed for $5 \mathrm{~min}$. Once bowel perfusion confirmed, transection made and anastomosis done via standard protocol. Anastomotic complication defined as dehiscence or stricture within 30 days, reflecting disruption of anastomotic integrity or ischemia. Results Colorectal cancer (95.2\%) was the most prevalent indication for the surgery. Splenic flexure mobilization was performed in $30.4 \%$. Overall anastomotic complication rate was around $3.60 \%$ (1 patient (1.08\%) with ICG A group vs 6 patient $(6.06 \%)$ with control group). In control group 3 patients underwent resurgery for anastomotic complication ( 2 patient with permanent stoma and 1 patient with Redo anastomosis). Anastomotic Leak patient in ICG Group was managed conservatively with Diversion loop ileostomy and exactly two months later ileostomy closed without any morbidity. There was no AL in the 15 patient $(15.78 \%)$ who had a change in the surgical plan based on the intraoperative perfusion assessment with ICG A group. Conclusion ICG A provide useful information about the vascular perfusion during colorectal surgery and may lead to change in the site of resection and or anastomosis, possibly affecting Anastomosis leak rate.

\section{MANAGEMENT OF NEGLECTED HIRSCHSPRUNG'S DISEASE IN ADULTS AND ADOLESCENTS}

\section{Sunita Ojha \\ Santokba Durlabhji Memorial Hospital and Medical Research Institute, Jaipur, IN}

Background/Aim Hirschsprung's disease in adolescent and adults is rare and usually neglected resulting in often misdiagnosed cause of refractory constipation and distal colonic physiologic obstruction and may experience rapildly worsening constipation. We present our experience of Hirschsprung's disease in adolescent and adults. AIMS: To analyse patients of Hirschprung's disease (HD) presenting from 14 years of age onwards and study the outcome. Methods Patients presenting at age of 14 years and beyond for HD from 2008 to 2018 were included in study. Clinical features, investigations, type of surgery, postoperative course, complications, and follow up was studied. Results Of all patients managed for HD from 2008 to 2018, 16patients presented between 14 and 30 years of age. All presented with constipation requiring enemas, and multiple admissions. All had barium enema suggestive of Hirschsprung's disease and were biopsy proven. Four patients were diagnosed HD at other center during their childhood but parents were not willing for colostomy so refused surgery. At our institute, two of these patients underwent primary Duhamel, and one underwent single stage Swensons procedure. One presented with severe anemia, respiratory distress. We could not operate this patient as he succumbed to severe morbidity and sepsis. Three patients had undergone transverse loop colostomy at other centre, colonic muscular hypertrophy persisted, requiring conversion to divided sigmoid colostomy and later Duhamel's procedure. Four cases presented with intestinal obstruction, requiring colostomy, and later definitive pull through (One Duhamel, two Swenson's). Five patients underwent primary (without colostomy) laparoscopic Swenson's procedures. All patients were followed up till one year postoperatively. Conclusion Hirschsprung's disease should be excluded in all patients presenting with persistent constipation. In patients with obstruction, massively dilated and hypertrophied colon, staged reconstruction (colostomy, then definitive surgery) may be needed. Divided stoma is recommended compared to loop stoma, as it offers better diversion and decompression. In cases without obstruction, with proper selection of cases, laparoscopic or single stage surgery (without colostomy) can be done with good results.

\section{CLINICAL VALIDATION \\ OF THE COMPREHENSIVE COMPLICATION INDEX IN COLON CANCER SURGERY}

\author{
Nicolo Tamini, Davide Bernasconi, Lorenzo Ripamonti, Giulia Lo \\ Bianco, Marco Braga, Luca Nespoli
}

Asst Monza, Ospedale San Gerardo, Monza, IT

Background/Aim The Comprehensive Complication Index (CCI) was recently introduced to overcome the potential limitations of the Clavien-Dindo Classification (CDC) in reporting surgical morbidity. To date, the sensitivity of the CCI in a homogeneous cohort of colonic resections for oncologic purposes has not been reported. The present study aimed to compare the CCI with the conventional CDC in colon cancer patients. Methods The clinical data of patients submitted to an elective colectomy for adenocarcinoma were retrieved from a prospectively maintained database. Postoperative complications and length of stay were reviewed, and CDC and CCI scores were calculated for each patient. The association of the CCI and the CDC with the length of stay, prolongation of stay and readmission rate were assessed and compared. Results In a cohort of 770 patients, the overall postoperative morbidity was $26.9 \%$. In particular $157(20.4 \%)$ patients had more than one complication. A strong correlation between the two scoring system was observed $(\mathrm{r}=99.4 \%$; $95 \% \mathrm{CI}$ 99.3-99.5\%). In multivariate analysis, both the CDC and CCI showed a strong association with length of stay (LOS), prolonged LOS and readmission: however CCI had a higher predictive ability for all endpoints. Regarding subgroup analysis, the difference between the $\mathrm{CCI}$ and CDC was progressively increased when evaluating outcome measures in complicated and multi-complicated patients. Conclusion The CCI adequately reports the overall burden of postoperative complications in patients who underwent colonic resection for cancer. The CCI showed a greater ability than the CDC to predict hospital stay, particularly in patients with multiple postoperative complications.

\section{LIFT VS ERAF IN PATIENTS WITH HIGH FISTULA IN ANO (FRAIL TRIAL): INTERIM ANALYSIS}

Pankaj Kumar, Siddhant Sarthak, Tushar Mishra, Prakash Kumar
Sasmal

AIIMS Bhubaneswar, Bhubaneswar, IN

Background/Aim This study aimed to compare the postoperative outcome and success rate of Endorectal advancement flap (ERAF) and Ligation of Intersphincteric Fistula Tract (LIFT) in high-type patients fistula in ano. Methods Patients aged more than 18 years with high fistula in ano of cryptogenic origin were included in this double-blind (participants and the assessor), randomized control trial. The primary endpoint was complete healing of the fistula at the end of 6 months. The Wexner score measured continence at the end of 1 and 6 months. Postoperative pain was assessed at the end of the 1st week, 1 st month, and 6 months. Quality of life was evaluated at the end of 6 months. Perioperative complications, duration of surgery, and hospital stay in hours were also calculated. Results A total of 84 patients were recruited (42 in each group). The majority of the patients were male ( 38 in LIFT vs. 39 in ERAF). Sixty patients have 
completed six months of follow-up. LIFT's ealing/success rate was significantly better than the ERAF group $(80 \%$ vs. $53.3 \%$, p value $<0.05)$. Three patients in ERAF and one in the LIFT group had flatus incontinence at the end of one month. The VAS score at the end of the first week and quality of life at 6 months was significantly better in the LIFT group $(3.7 \pm 1.6$ vs. $4.7 \pm 0.81, \mathrm{p}<0.05$ and $0.62 \pm 0.2$ vs. $0.5 \pm 0.2, \mathrm{p}<0.05)$. The mean duration of surgery was significantly less in the LIFT group $(44.16 \pm 8.27 \mathrm{~min}$ vs. $88.16 \pm 10.60$ ). None of them had any postoperative complications, and more than $80 \%$ of them were discharged within $24 \mathrm{~h}$. Superficial wound dehiscence was observed in $86.6 \%$ of patients in the ERAF group and $73.3 \%$ in the LIFT group. Conclusion The results suggest better healing, shorter operative duration, less immediate postoperative pain, and better quality of life at 6 months in the LIFT group.

\section{IMPACT OF DIABETES ON OUTCOMES POST RIGHT HEMICOLECTOMIES (RH)}

\section{Sarit Badian, David Goltsman, Chris Berney}

\section{Bankstown Hospital, Sydney, AU}

Background/Aim There is limited data on the impact of diabetes following right hemicolectomies ( $\mathrm{RH}$ ) for cancer resections. The aim of this study was to determine the significance of insulin dependent (IDDM) and non-insulin dependent (NIDDM) diabetes on the postoperative outcomes (medical and surgical complications) following RH. Methods Data was obtained from the ACS-NSQIP database between 2014 and 2018. Logistic regression models were used to examine the association between diabetic status (IDDM and NIDDM versus non diabetic) and medical or surgical complications. The statistic of interest in these analyses was the odds ratios [95\% (CI)] and their p-values of the IDDM and NIDDM groups when compared with the nondiabetic group. Subgroup analyses were performed based on method of resection: open, minimally invasive (laparoscopic/ SILS) and robotic. RH commenced laparoscopically or robotically, but converted to open were overall classified as open procedures. Results A total of 18,889 RH patients were identified out of which, $10,861(57.5 \%)$ underwent a laparoscopic approach, $6657(35.2 \%)$ an open procedure and $1,381(7.3 \%)$ a robotic resection. Twenty percent $(3800 / 18,899)$ of the patients had diabetes. Of these, $2,512(66 \%)$ were NIDDM and 1288 (34\%) were IDDM. The majority of patients $(74.11 \%)$ were aged between 60 and 89 years. A total of 4475 patients $(23.6 \%)$ suffered postoperative complication. Regression analyses identified patients with IDDM to have a higher likelihood of medical s (OR 1.28, p-value 0.006) or surgical complications (OR 1.40 , p-value $<0.001)$. In the subgroup analysis, IDDM mostly affected outcomes in the minimally invasive approach, increasing the odds of a medical (OR 1.33, p-value 0.04) or surgical complications (OR 1.56, p-value < 0.001). Conclusion This outcome-based study demonstrates that patients diagnosed with right-sided colon cancer and suffering from IDDM have an increased relative risk of developing major post-surgical complications of 30-40\%, compared to non-diabetics. Furthermore, this complication rate is further raised following minimally invasive procedures as compared to open approach.

\section{MAPPING OF AETIOLOGIES AND CLINICAL PRESENTATION OF ACUTE COLITIS AND DEVELOPMENT OF A DIAGNOSTIC MANAGEMENT ALGORITHM: RESULTS FROM A LARGE PROSPECTIVE STUDY IN A TERTIARY CENTRE}

Jeremy Meyer Geneve, Jacques Schrenzel, Alexandre Balaphas, Vaihere Delaune, Mohamed Abbas, Philippe Morel, Giacomo Puppa, Laura Rubbia-Brandt, Philippe Bichard, Jean-Louis Frossard, Christian Toso, Nicolas Buchs, Frédéric Ris

Hôpitaux Universitaire de Genève (HUG), Geneve, $\mathrm{CH}$

Background/Aim Our objective was to describe the aetiologies of acute colitis and to identify patients who require diagnostic endoscopy. Methods Patients with symptoms of gastrointestinal infection and colonic inflammation on computed tomography were prospectively included. Those immunosuppressed, with history of colorectal cancer or inflammatory bowel disease (IBD) were excluded. Stools were screened with BD-Max and BioFire FilmArray GI panel. Faecal calprotectin was determined. Patients with negative BD-Max underwent colonoscopy. The study was registered into clinicaltrials.gov (NCT02709213). Results One hundred and seventy-nine patients were included. BD-Max was positive in 93 patients (52\%) and FilmArray in 108 patients $(60.3 \%)$. Patients with infectious colitis $(\mathrm{n}=103,57.5 \%)$ were positive for Campylobacter spp $(\mathrm{n}=57$, $55.3 \%)$, Escherichia coli spp $(\mathrm{n}=8,7.8 \%)$, Clostridium difficile $(\mathrm{n}=23,22.3 \%)$, Salmonella spp $(\mathrm{n}=9,8.7 \%)$, viruses $(\mathrm{n}=7,6.8 \%)$, Shigella spp $(n=6,5.8 \%)$, Entamoeba histolytica $(n=2,1.9 \%)$ and others $(n=4,3.9 \%)$. Eighty-six patients underwent colonoscopy, which was compatible with ischemic colitis in 18 patients $(10.1 \%)$ and IBD in 4 patients (2.2\%). Among patients with negative FilmArray, a faecal calprotectin $>625 \mu \mathrm{g} / \mathrm{g}$ allowed identifying patients with IBD with an area under ROC curve of $85.1 \%$. Introduction of a diagnostic management algorithm including FilmArray and faecal calprotectin could allow decreasing unnecessary colonoscopies from 82 to 29 (corresponding to a decrease of 64.6\%). Conclusion Computed tomography-proven colitis was mostly of infectious aetiology. Diagnostic management of patients with acute colitis should include broad molecular testing of the stools and, in patients with a calprotectin concentration $>625 \mu \mathrm{g} / \mathrm{g}$, colonoscopy to exclude IBD.

\section{ROLE OF EENTEROBIUS VERMICULARIS INFESTATION IN SURGICALLY REMOVED APPENDICES WITH A CLINICAL DIAGNOSIS OF ACUTE APPENDICITIS: ANALYSIS OF 1742 PATIENTS}

Rocio Santos Rancaño, Mariela Milagros Delgado Morales, Miguel Hernández Garciá, Maria del Mar De Elena Soto, Víctor Sánchez. Bueno, Carlos Cerdán Santacruz, Ramón Alonso Guillén

Hospital Comarcal de Melilla, Melilla, ES

Background/Aim The aim of this study is to describe the incidence of Enterobius vermicularis in a series of patients that underwent an appendectomy due to clinical suspicion of acute appendicitis, and to evaluate the histopathological findings in appendectomy specimens with this parasite infestation in order to deduce a causal relationship. Methods We have studied retrospectively 1742 patients who have undergone an appendectomy at our Hospital due to the preliminary 
diagnosis of appendicitis, between January 2000 and September 2020. They have been evaluated in terms of age, gender, and pathological findings. Diagnosed cases of parasitosis were histopathologically reassessed to look for the presence of an inflammatory response. These results and the operative findings were reviewed individually for each patient. The statistical analysis was performed with the IBM SPSS version 21 software. Results A total of 1742 appendectomies for clinical suspicion of appendicitis were performed. Enterobius vermicularis infestation was identified in 19 cases. Histological confirmation of acute appendicitis was obtained in 1683 patients. The incidence of Enterobius was 1.09\% (19/1742) in patients with clinically suspected appendicitis and $1.12 \%$ (19/1683) in histologically confirmed appendicitis. The female:male ratio in the parasite infestation was $1: 1$ and the median age was 11 years (interquartile range 9-21). In our series, 9 patients (47.4\%) had lymphoid hyperplasia, 6 (31.6\%) had a normal structure, $4(21 \%)$ had findings of acute transmural inflammatory infiltrate (acute appendicitis) and in 6 cases fecaliths were evidenced in addition to pinworms. Acute inflammation was described in 6 patients $(37.5 \%)$ with only one case of gangrenous appendicitis. Conclusion Enterobius vermicularis infestation appears to be an incidental finding in appendectomies rather than a cause of appendicitis, but it probably stimulates lymphoid hyperplasia, which can clinically mimic acute appendicitis. Including this parasite in the differential diagnosis of appendectomy is important to avoid unnecessary appendectomies and associated morbidity, and to apply anthelmintic treatment.

\section{MAGNIFYING CHROMOENDOSCOPY FOR SELECTING PATIENTS WITH RECTAL TUMORS TO LOCAL EXCISION}

\author{
Cintia Mayumi Sakurai Kimura, Fabio Shiguehissa Kawaguti, \\ Natally Horvat, Caio Rizkallah Nahas, Carlos Frederico S. Marques, \\ Ulysses Ribeiro-Junior, Sergio Carlos Nahas, Fauze Maluf-Filho
}

Hospital das Clinicas da Faculdade de Medicina da Universidade de Sao Paulo, São Paulo, BR

Background/Aim Adequate staging of large rectal adenomas and early adenocarcinomas is often a challenge, since overstaging may lead to radical surgery with total mesorectal excision (TME) for patients who could have been treated with local excision. Endorectal ultrasound has several limitations and is less accurate for large lesions. MRI is often used in these cases but tends to overstage large precocious lesions. Magnifying chromoendoscopy (MCE) has been successfully used in Eastern countries to predict depth of invasion of colorectal neoplasms. Thus, the aim of this study was to evaluate the ability of MCE to stage rectal sessile polyps ( $>20 \mathrm{~mm}$ ), laterally spreading tumors ( $>20 \mathrm{~mm}$ ), and depressed lesions (of any size) in a single Western center. Methods From 2011 to 2020, 48 consecutive rectal lesions that were assessed by MCE according to Kudo pit patterns were retrospectively reviewed, and only lesions with enbloc resection were included. MCE reports were compared with the pathological reports of the specimens. Results The average size of the lesions was $71 \mathrm{~mm}(\mathrm{SD} \pm 42.2)$, and $37 / 48(77 \%)$ of them had intramucosal or submucosal invasion ?1 $\mu \mathrm{m}$. MCE classified $83 \%$ of the lesions as Kudo IV or Vi (non invasive), and 17\% as Vn (massive invasion). The sensitivity of MCE to predict deep submucosal invasion was $63.4 \%$ (95\% CI $30.8-89.1 \%$ ), the specificity was $97.3 \%$ (95\% CI 85.8-99.9\%), positive and negative predictive values were $87.5 \%$ (95\% CI $49.1-98.1 \%$ ) and $90.0 \%$ (95\% CI $80.4-95.2 \%$ ), respectively. Cohen's kappa was 0.7 (substantial agreement). Conclusion As the specificity and negative predictive value of MCE were high, patients who are negative for deep submucosal invasion on
MCE can be safely sparred of TME. The lower sensitivity resulted in some patients with massive submucosal invasion being referred to local excision. Fortunately, those patients could still be salvaged with timely TME. Further investigations with larger prospective cohorts are needed to determine whether MCE is superior to endorectal ultrasound or MRI to evaluate large rectal adenomas and early adenocarcinomas.

\section{HIGH-RISK HPV AS PREDICTOR OF PROGRESSION TO HIGH-GRADE DYSPLASIA AND RECURRENCE AFTER TREATMENT}

Cintia Mayumi Sakurai Kimura, Sergio Carlos Nahas, Edesio Vieira Silva-Filho, Vinicius Lacerda Ribeiro, Flavio Ferraz de Paes Alcântara, Aluisio Cotrim Segurado, Ivan Cecconello, Sergio Carlos Nahas

Hospital das Clinicas da Faculdade de Medicina da Universidade de Sao Paulo, BR

Background/Aim It is well recognized that infection with carcinogenic HPV strains is responsible for up to $90 \%$ of cases of anal squamous cell carcinoma, with a strong predominance of subtypes 16 and 18. The aim of this study is to evaluate whether patients with HPVs 16/18, or other high-risk subtypes have increased risk of progression to or recurrence of high-grade squamous intraepithelial lesions (HSIL). Methods Patients who were followed for at least 3 months with HPV test and high-resolution anoscopy (HRA) in a single institution were included. All patients who had HSIL were routinely treated with cauterization and re-evaluated with HRA for surveillance. Results 186 patients (78\% HIV +, 71\% male) were included. The average follow-up was 11.4 months $(\mathrm{SD} \pm 4.4)$. At baseline, $19 \%$ were positive for HPV 16/18, 35\% for other high-risk HPV (HR-HPV), and 46\% were negative for any HR-HPV. The initial prevalence of HSIL was 34/186 (18\%), among which $11(32 \%)$ had HPV 16/18, and $14(41 \%)$ had other HR-HPV. None of the patients who initially had HSIL and no HR-HPV presented with recurrent HSIL on follow-up. On the other hand, progression to or recurrence of HSIL was observed in 39\% of patients who had HPV 16/18 at baseline, $21 \%$ of patients who had other HR-HPV at baseline, and 5\% of patients without HR-HPV at baseline had progression to HSIL $(\mathrm{p}<0.01)$. No difference was observed in progression or recurrence of HSIL among HIV + (16\%) and HIV-negative (20\%) patients $(\mathrm{p}=0.85)$, nor between men $(18 \%)$ and women $(15 \%), \mathrm{p}=0.83$. The odds ratio for progression or recurrence of HSIL was 12.9 for patients with HPV 16/18 (95\% CI 3.8-43.1), and 4.7 for patients with other HR-HPV (95\% CI 1.4-13.9). Conclusion Infection with HR-HPV can be interpreted as an indicator of worst outcomes, since it increases the risk of progression to HSIL, and recurrence of HSIL after treatment, especially subtypes 16 and 18 . As patients who were negative for HRHPV at baseline rarely presented with HSIL on further evaluation in the period studied, it is likely that these patients could be screened at larger intervals than patients with HR-HPV. Further research with larger cohorts and longer follow-up are warranted. 


\section{INFLAMMATORY MYOFIBROBLASTIC TUMOR PRESENTING AS ILEOCECAL INTUSSUSCEPTION}

Stefano Lafranceschina, Marialessia Milella, Anna Stella Lippolis, Vito Dagostino

Ospedale San Paolo, Bari, IT

Background/Aim Inflammatory myofibroblastic tumor (IMFT) is an uncommon mesenchymal solid tumor. It predominantly affects the pediatric age group and commonly involves the lungs. However, extrapulmonary involvement has also been reported. We report a rare case of IMFT causing ileocolic intussusception leading to acute intestinal obstruction in an adult. Methods A 77-year-old Caucasian woman presented to the emergency department with a ten days history of colicky abdominal pain, associated with diarrhoea. Computer tomography (CT) showed an intussusception at the cecum associated with wrapping of the last ileal tract. Laparotomy confirmed an ileocecal intussusception: the lead point of intussusception (a welldefined $6 \times 5 \times 5 \mathrm{~cm}$ solid mass), was found at $5 \mathrm{~cm}$ proximal to the ileocecal junction. A right hemicolectomy with oncological criteria and ileo-transverse anastomosis was performed. Results Postoperative course was uneventful and the patient was discharged on the seventh postoperative day. Histopathological examination revealed an ileocecal IMFT with exophytic growth. IMFT usually affects lungs with rare gastrointestinal involvement. Clinical presentation is related with location of the neoplasm. Intussusception in adults presents with non-specific symptoms and classical CT signs can facilitate preoperative diagnosis. However IMFT diagnosis is histopathological which usually implies surgical resection: as known from the literature, about $60 \%$ of patients with ileocecal intusception is linked to the presence of neoplasia and $50 \%$ of these are malignant. Based on these data and on the impossibility of preoperatively CT diagnosis of IMFT, it is always recommended to perform a complete resection as in our case, even in urgency. Conclusion IMFT causing ileocolic intussusception with acute intestinal obstruction is an extremely rare presentation of an uncommon entity in adults. High index of suspicion at CT scan depending on clinical presentation can result in prompt diagnosis and early management. The main prognostic indicator being the adequacy of the primary excision: complete surgical excision is the mainstay of the treatment and provides the best chance to limit recurrence.

\section{DIFFERENT FORMS OF PRESENTATION: FROM SIMULATING AN ADENOCARCINOMA TO ITS PRESENTATION AS AN INTESTINAL OBSTRUCTION}

\author{
Mariela Milagros Delgado Morales, Roció Santos Rancaño, Alberto \\ García García, Beatriz Garciá Del Pino, Nestavo De Los Reyes \\ Lopera, Juan Francisco Guadalajara Jurado
}

\section{Infanta Elena Hospital, Huelva, ES}

Background/Aim Colorrectal neuroendocrine tumors are a group of heterogeneous neoplasms which are divided into low-grade or carcinoids, and high-grade neuroendocrine tumors, which are aggressive. The most frequent anatomical location is the cecum, rectum and sigmoid. Their clinical presentation depends on the primary site and many are discovered incidentally, either during screening or during the investigation of nonspecific abdominal complaints. Methods The first case is a 70-year-old female patient who presented to the emergency department with an intestinal obstruction. An abdominal
CT showed an hypercaptant intraluminal lesion at the ileocecal valve, compatible with a carcinoid tumor of the terminal ileum/cecum. The second case is a 62-year-old man who underwent a screening colonoscopy. During the procedure, the endoscopist removed several polyps, one of them at $10 \mathrm{~cm}$ from the anal margin, which was reported as a well-differentiated adenocarcinoma over an adenovillous polyp that infiltrated the submucosa. Pelvic MRI revealed a neoplasm of the middle rectum. Results In the first case, we performed an urgent laparotomy finding a stenosing tumor at the ileocecal valve/cecum (palpable intraluminally) with an umbilication in the surface which compromised the terminal ileum, ileocecal valve and cecum, and caused traction of the meso at this level. We performed an oncological right hemicolectomy. The anatomopathological study of the specimen revealed a Grade 1 Neuroendocrine Tumor. The IHQ determined positivity for: CQ, chromogranin and CD 56. In the second case, we performed a laparoscopic low anterior resection. The pathological study reported a Grade 1 Neuroendocrine Tumor. The IHQ study determined positivity for: chromogranin, synaptophysin, CD56, CAM 5.2 and pancytokeratin. CD X-2 negative. Both patients are under follow-up by oncology and with no signs of long-term recurrence. Conclusion Low grade tumors or carcinoids have a benign behavior and a good survival. Their clinical presentation does not differ.

\section{CIRCUMFERENTIAL RESECTION MARGIN IS A PREDICTOR FOR METASTATIC DISEASE AFTER RECTAL CANCER SURGERY: A NATION- WIDE POPULATION-BASED STUDY COHORT}

\author{
Erik Agger, Fredrik Jörgren, Marie-Louise Lydrup, Pamela \\ Buchwald
}

Dept of Surgery, Skaine University Hospital, Malmö, SE

Background/Aim To evaluate circumferential resection margin $(\mathrm{CRM})$ as a risk factor for distant metastasis (DM) in rectal cancer. The treatment of rectal cancer has evolved over the last decades. Surgical radicality is the single most important factor in preventing recurrences, both locally and distantly. CRM $\leq 1.0 \mathrm{~mm}$ is considered to increase recurrence risk. However, not all patients with CRM $\leq 1.0 \mathrm{~mm}$ suffer recurrence. This study explores the risk of DM in relation to exact CRM. Methods All patients treated with abdominal resection surgery for rectal cancer between 2005 and 2013 in Sweden were eligible for inclusion in this retrospective study. Primary endpoint was DM. Results 12,146 cases were identified. 8593 cases were analysed after exclusion. $718(8.6 \%)$ patients had CRM $\leq 1.0 \mathrm{~mm}$ and $7601(91.4 \%)$ patients CRM $>1.0 \mathrm{~mm}$. DM rate was $36.9 \%$ $(\mathrm{n}=56 / 153), 29.2 \%(\mathrm{n}=165 / 565), 25.2 \%(\mathrm{n}=26 / 103)$ and $18.5 \%$ ( $\mathrm{n}=1385 / 7498)$ when CRM was $0.0 \mathrm{~mm}, 0.1-1.0 \mathrm{~mm}, 1.1-1.9 \mathrm{~mm}$ and $\mathrm{CRM} \geq 2 \mathrm{~mm}$ respectively. Multivariable analysis revealed higher DM risk in CRM $0.0 \mathrm{~mm}$ versus $\geq 2.0 \mathrm{~mm}$ (HR 1.73, 95\% c.i. 1.30 to $2.29 ; \mathrm{P} \leq 0.001)$ and in $\mathrm{CRM} 0.1-1.0 \mathrm{~mm}$ versus $\geq 2.0 \mathrm{~mm}$ (HR $1.29,95 \%$ c.i. 1.09 to $1.53 ; \mathrm{P} \leq 0.001$ ). No significant difference in DM risk in CRM 1.1-1.9 mm versus $\geq 2.0 \mathrm{~mm}$ (HR 1.10, 95\% c.i. 0.74 to $1.64 ; \mathrm{P}=0.623$ ) could be detected. Conclusion The risk of DM decreases with increasing CRM. Moreover, CRM $<2.0 \mathrm{~mm}$ is an independent, significant risk factor for DM. CRM should be considered when predicting risk of DM after rectal cancer surgery. 


\section{PERIANAL ABSCESS MICROBIOLOGY SWABS DO NOT IMPACT CLINICAL MANAGEMENT-A RETROSPECTIVE COHORT STUDY}

\author{
Adam O'Connor, Salford Quays, Shariq Sabri, Sana Ullah \\ Wrightington Wigan and Leigh NHS Trust, Salford Quays, GB
}

Background/Aim The purpose of our study is to assess whether microbiology swabs taken during incision and drainage of perianal abscesses in our hospital have any impact on the post-operative clinical course. We are also assessing the demographics of perianal abscesses locally and the microbiology present. Methods Data from emergency operative theatre lists were interrogated for patients undergoing incision and drainage of perianal abscess in the period March 2019-March 2020. Each electronic medical record for patients who had perianal abscess surgery was interrogated for age, sex, smoking status, diabetic status, whether or not microbiology swab was taken for analysis, what organism was grown and any post-op fistula development. Results 71 patients met inclusion criteria. 55 (77.5\%) patients had pus swabs sent at incision and drainage for microbiological analysis. Of the 55 patients swabbed, 20 (36\%) had no organism growth, $22(40 \%)$ had anaerobes growth-the most common organism in this study. Of all 55 swabs sent, only $14(25 \%)$ were acknowledged. The presence of smoking $(\mathrm{p}<0.05)$ was statistically significantly associated with development of fistula-in-ano longer term. Conclusion Currently, our data may indicate that microbiology swabs do not influence management of patients with acute perianal abscess. There appears to be discrepancies in whether swabs are taken and acted upon depending on individual surgeon preference. There is no apparent significant group of organisms that indicate future fistula development. We would suggest larger, national scale studies to further explore our conclusions.

\section{RESULTS OF EXTRACORPOREAL AND INTRACORPOREAL ANASTOMOSIS IN LAPAROSCOPIC RIGHT HEMICOLECTOMY PERFORMED BECAUSE OF MALIGNANT TUMOUR}

\author{
László Sikorszki Kecskemét, Kornélia Trepák, Ildiko Horti, Kornél \\ Vajda
}

Bács-Country Hospital, Kecskemét, HU

Background/Aim Laparoscopy became evident for right-sided colon surgery too. Today the laparoscopic-assisted right-hemicolectomy is the gold-standard with extracorporeal anastomosis. Morbidity according to randomized trials is still approxymately $30 \%$. The developement of technique rendered to make intracorporeal anastomosis. Our aim was to compare the short-term results of the two methods. Methods To analyse the short-term results of right-sided hemicolectomy that were performed because of malignant tumours with the comparison of the two methods. A cohort of 184 right-sided haemicolectomy were performed from 01.01.2018 to 31.12.2019 from which 122 were operated on because of a malignant disease. 51 open and 71 laparoscopic operations were performed. Results The average age of 37 men and 34 women were 70, 59 and 72, 14 years, respectively. 50 patients underwent extracorporeal (EA) anastomosis and 21 intracorporeal (IA) anastomosis. Whithin 30 days the number of complications were 18 in the EA group and 3 in the IA group $(\mathrm{p}=0.067) .3$ from the EA group and 1 from IA group died whithin 30 days $(p=0.66)$. The average lenght of stay were 9.48 days in the EA group and 6.52 days in the IA group together with the complicated cases $(p=0.001)$ while 6.35 days and 5.55 days without the complicated cases $(\mathrm{p}=0.09)$. The average duration of operation was $147 \mathrm{~min}$ in the EA and $146.47 \mathrm{~min}$ in the IA group $(\mathrm{p}=0.11)$. Conclusion We found concordance with the literature that there are fewer complications in case of IA which might be related to shorter lenght of stay. There is no significant difference between the operation times. Bearing these facts in mind, IA might be suggested for right-sided laparoscopic hemicolectomy.

\section{COLONIC VERSUS RECTAL GUNSHOT INJURIES, IN TERMS OF POSTOPERATIVE OUTCOMES}

\author{
Salah Mansor Benghazi, Monir Alfitory, Ahmed Algialany, Ayoop \\ Buzaja
}

Al-Jalaa teaching hospital, Benghazi University, Benghazi, Libya, LY

Background/Aim In the current time, gunshot injuries are considered common emergency cases and have become a significant problem globally. There is clear evidence that, because of its anatomical features, the colon is commonly injured in abdominal gunshot wounds, and this has a profound clinical and surgical significance because of the risk of septic complications. Our objective is to assess and analyze the differences between colon versus rectal gunshot injuries in terms of postoperative outcomes to determine which type of injury is more lethal. Methods A Cohort study to compare patients who had colon gunshot injuries, and others who had rectal gunshot injuries, to analyze the difference in the influence of the anatomical site of injury on the postoperative complications and mortality rate, in all patients, who underwent urgent explorative laparotomy due to trunk gunshot injuries, with colon and rectal penetrating wounds, in our hospital from February 2011 to December 2016. The primary endpoint of the study was the appearance of postoperative complications, while the secondary endpoint was the finishing of two years of follow-up for all patients. Results During the study period, we have done 192 urgent exploratory laparotomies for patients who were suffered from trunk gunshot injuries, with colon and rectal penetrating wounds. $186(96.6 \%)$ were males and $6(3.1 \%)$ were females. The mean age was 29.03 years. $164(85.4 \%)$ patients who had colon gunshot wounds which considered the first group in the study, while in the second group were $28(14.5 \%)$ patients who had rectal gunshot wounds. The postoperative complications were occurred in 56 (34.1\%) patients in the first group and seven $(25 \%)$ patients in the second group, while the mortality was $29(17.6 \%)$ and nine $(32.1 \%)$ patients in the first and second group, respectively. Conclusion In our study, there was no significant statistical difference between the site of injury, whether it is in the colon or rectum, and the rate of postoperative complications. $(\mathrm{P}=0.340)$, as well as the mortality rate $(\mathrm{P}=0.075)$.

\section{LASER SCLEROTHERAPY OF HEMORRHOIDS: AN EFFECTIVE MINIMAL INVASIVE TREATMENT OF GRADE1}

\section{Jaya Maheshwari}

Jyoti Hospital, Jaipur, Rajasthan, IN

Background/Aim Hemorrhoidal disease is ranked first amongst diseases of the rectum and large intestine. The estimated prevalence ranges from 2.9 to $27.9 \%$ worldwide. The treatment options for symptomatic hemorrhoids have varied over time. According to ASCRS Guidelines for Management of Hemorrhoids (2010), there is 
a strong recommendation based on moderate-quality evidence that early grades including grade I, II and even III that do not respond to conservative treatment can be managed with office procedures, which aim to decrease blood flow to the hemorrhoid, reduce the redundant tissue and fix the hemorrhoid to the underlying tissue to reduce prolapse. We used diode laser energy with hemorrhoidal arterial ligation to treat the grade 1,2 and 3 hemorrhoids. Methods 51 patients from year 2018 to 2020 having grade 1, 2 and 3 hemorrhoids were treated with Laser sclerotherapy and Hemorrhoidal artery ligation. The procedure was performed in Epidural Anaesthesia. The patients in modified lithotomy position, the conical tip fibre of $1470 \mathrm{~nm}$ laser was used. The fibre was introduced in the submucosal plane of each hemorrhoid and energy delivered upto $250 \mathrm{~J}$ per pile mass. The fibre was continuously moved back forth and sideways to avoid it sticking to one point. This was followed by Hemorrhoidal artery ligation with vicryl 2-0.Maximum of 3 hemorrhoids were treated in each patient. Ice was applied in anal canal after the procedure. The post-operative period was uneventful and the patients were discharged in $24 \mathrm{~h}$ on a Vas scale of 2 . Results The postoperative period was uneventful and the patients were discharged in $24 \mathrm{~h}$ on a Vas scale of 2 . Conclusion Patients with grades 1, 2, 3 hemorrhoids can be treated with medical treatment or office procedures. Hemorrhoidal artery ligation is a potential non-excisional technique for the treatment of grades II-III hemorrhoids with minimal postoperative pain and quick recovery. Laser sclerotherapy is a recent laser procedure for outpatient treatment of hemorrhoids in which hemorrhoidal arterial flow feeding the hemorrhoidal plexus is stopped by laser coagulation and Hemorrhoidal artery ligation. This procedure offers shorter operative time, less post-operative pain and less time off work.

\section{DOES PREOPERATIVE STENT POSITIONING IN OBSTRUCTIVE LEFT SIDED COLON CANCER INCREASE THE RISK OF PERINEURAL INVASION?}

Nicolò Tamini, Luca Cigagna, Marco Angrisani, Massimo Oldani, Luca Nespoli, Marco Braga, Luca Gianotti

\section{Università degli studi di Milano bicocca, Portogruaro, IT}

Background/Aim Colonic stenting as a bridge to surgery has been shown to be a safe and effective treatment for left-sided malignant colonic obstruction depending on local expertise. However, concerns still exist regarding its oncological safety. In particular, several reports showed an increased prevalence of perineural tumor invasion following stent placement. Methods We retrospectively reviewed 114 patients presenting with left-side obstructing colon cancer over a 10 -years period. The relationship between perineural invasion and colonic stenting was analysed using univariate and multivariate analyses. Results Perineural invasion was found to be strongly associated with pathological features, including TNM stage, $(\mathrm{p}<0.001)$, poor differentiation $(\mathrm{p}=0.002)$, vascular invasion $(\mathrm{p}<0.001)$, lymphatic invasion $(\mathrm{p}<0.001)$, whereas no significant association with preoperative stenting was observed $(\mathrm{p}=0.918)$ after performing univariate analysis. In the multivariate model only TNM stage III-IV (OR: $6.810,95 \%$ CI 1.972-23.518, p=0.002) and venous invasion (OR: $5.325,95 \%$ CI $1.911-14.840, \mathrm{p}=0.001$ ) were independently associated with perineural invasion. Conclusion The results of this study suggest no association between preoperative colonic stenting and perineural invasion.

\section{RAMAN SPECTROSCOPY A NEW METHOD FOR DETECTING DISEASE DURING ADJUVANT CHEMOTHERAPY FOR COLORECTAL CANCER?}

Susan Chandler Swansea, Cerys Jenkins, Freya Woods, Andrew Cunningham, Rhys Jenkins, Karen Phillips, Emma Christopher, Maung Moe, Vallipuram Vigneswaran, Peter Dunstan, Dean Harris

Swansea Bay University Health Board, Swansea, GB

Background/Aim There is current unmet need for a biomarker in colorectal cancer to monitor disease during adjuvant treatment. The response to treatment is currently reliant on computed tomography (CT) imaging at 3 or 6 months after treatment initiation combined with the poor sensitivity tumour marker carcinoembryonic antigen (CEA). Both modalities are unable to detect micrometastatic disease and do not lend themselves to more intensive monitoring of response; as such, disease progression despite chemotherapy can go undetected for months. Raman spectroscopy (RS) is an established accurate technology based upon the spectroscopic analysis of inelastically scattered light. It reflects the molecular and structural composition of the biological sample under investigation and has the shown the ability to detect colorectal cancer with $84 \%$ sensitivity and $91 \%$ specificity. This study will look at the use of serum RS during adjuvant chemotherapy for colorectal cancer and its potential use as a disease monitoring tool. Methods 400 patients will be recruited in 2 groups; group A patients with known metastatic disease receiving chemotherapy and group B patients receiving adjuvant chemotherapy to reduce the risk of recurrence following resection of the primary tumour. Serum blood samples will be obtained from patients at baseline, prior to each cycle of chemotherapy and at completion of treatment in parallel with current standard of care investigations CT and CEA. Raman spectra will be obtained from the serum at $532 \mathrm{~nm}$ and $735 \mathrm{~nm}$ laser sources and the spectra analysed. Results The primary outcome will be to detect spectral change in group A showing progression or reduction in size of metastases during treatment. In group B spectral changes which predate development of lymphatic, liver or lung metastases on standard imaging modalities will be sought. Recruitment for this study is currently underway and preliminary results have revealed distinct spectral changes in response to chemotherapy which will be presented. Conclusion Serum RS has shown the ability to detect colorectal cancer and given the current lack of tools for monitoring disease response during treatment there is a need to explore new techniques. As a blood based technique RS could provide a disease monitoring tool for patients that is reproducible, cost effective and readily acceptable to patients. The ability to detect recurrence earlier than current modalities would be invaluable to initiate different treatment plans and improve patient outcomes.

\section{THE VALUE OF CARDIOPULMONARY EXERCISE TESTING (CPET) IN PREDICTING POST- OPERATIVE MORBIDITY \& MORTALITY IN PATIENTS UNDERGOING COLORECTAL SURGERY-AN EXTENDED LITERATURE REVIEW}

Kelly Iles, Predrag Andrejevic

Mater Dei Hospital, Mosta, MT

Background/Aim The aim of this extended literature review is to critically appraise recent evidence regarding the efficacy of 
cardiopulmonary exercise testing in predicting post-operative morbidity and mortality in patients undergoing colorectal cancer surgery. Outcomes assessed shall include post-operative mortality, post-operative complications including surgical site infections, anastomotic leak, need for re-operation and unexpected admission to critical care as well as post-operative length of stay. Methods An extensive literature search was conducted in five main databases namely; Pubmed, Scopus, EMBASE, Web of Science and Cochrane databases for papers published between 2015 and 2020. The MeSH terms "cardiopulmonary exercise testing", "CPET", "colorectal cancer" and "colorectal cancer surgery" together with the boolean operator "+" were used to conduct this search. The search was extended to include papers published in the last ten years since an insufficient amount of studies were found in the initial search. A total of thirteen papers were deemed relevant to the scope of this literature review; eleven cohort studies and two systematic reviews one of which included a metaanalysis. Results Anaerobic threshold, peak oxygen consumption and ventilatory inefficiency are predictive of post-operative morbidity and mortality in patients undergoing elective colorectal cancer surgery. Conclusion Objective analysis of physiological fitness using cardiopulmonary exercise testing (CPET) is useful in detecting cardiopulmonary morbidities which may not otherwise have been noted during conventional preoperative assessment. Furthermore, threshold values for CPET-derived variables may prove useful in planning patient treatment and perioperative care.

\section{LOCAL APPLICATION OF AUTOLOGOUS PLATELET-RICH PLASMA IS SAFE AND EFFECTIVE IN TREATMENT OF COMPLEX CROHN'S PERIANAL FISTULAE-RESULTS FROM SINGLE CENTER PILOT STUDY}

\section{Dusan Podmanicky \\ University Hospital St. Michael's Hospital, Bratislava, SK}

Background/Aim Complex Crohn's perianal fistula (pCD) is a difficult-to-treat condition. Current therapeutic strategies are effective in up $50 \%$ of patients. Few preliminary reports suggest that autologous platelet-rich plasma (PRP) can enhance wound repair and may be effective in treating pCD, but conclusive results are lacking. Therefore, the aim of our study was to determine the efficacy and safety of autologous PRP in the treatment of $\mathrm{pCD}$. Methods A prospective, uncontrolled, single center study in a referral IBD center was conducted between July 2018 and February 2020. Adult patients (pts) with $\mathrm{pCD}$ failing on antibiotics, immune suppression and/or biologics were eligible for the study. All patients had non-cutting setons for a minimal period of 6 weeks prior study intervention. Autologous PRP was separated by centrifugation $60 \mathrm{ml}$ of peripheral blood in Harvest SmartPrep(C) System at the time of operation. Internal openings were closed by PDS 2/0 single suture and PRP was injected close to internal openings and fistula tracts. Patients were examined at outpatient clinic at week 1, month 1, 3 and 6 and any suspected sideeffects of the treatment were noted. Treatment effect was assessed by perianal Crohn Disease Activity Index (PCDAI assessed at baseline, month 1,3,6) and van Assche MRI score (assessed at baseline and month 6). The primary end-point was complete healing at month 6 defined as closure of all external fistula openings and absence of abscess on MRI. Results During the inclusion period, 21 pts with pCD were included (mean age 37 [21-61] years; 11 men). The majority of pts were using antiTNF biologics ( 9 adalimumab, 7 infliximab), 2 pts were treated by ustekinumab, one by vedolizumab, two pts were on immunomodulators monotherapy. The Only side effect was minor local pain at the injection site and perianal soiling during maximum of seven days following intervention. By May 2020, 19 pts finished the 6 months follow-up. Out of these 19 pts, fifteen (79\%) reached the primary end-point of complete healing. Baseline PCDAI (median 5, range 2-9) decreased significantly as early as at month 1 (median 1 , range $0-5 ; \mathrm{p}<0.001$ ) and remained further stable over 6 months. Van Assche MRI score decreased significantly from median of 13 (range 8-20) at baseline to 10 (range 4-18) at month $6(\mathrm{p}<0.001)$. Conclusion Local application of autologous platelet-rich plasma is safe and effective in inducing complete healing of difficult-to-treat complex Crohn's perianal fistulae.

\section{ELEVATED EXPRESSION OF PD-L1 IS ASSOCIATED WITH MICROSATELLITE INSTABILITY AND PREDICTS POOR SURVIVAL IN MIDDLE EASTERN CRC PATIENTS}

\author{
Khawla Al-Kuraya, Abdul Khalid Siraj, Sandeep Kumar \\ Parvathareddy, Fouad Al-Dayel, Alaa Abduljabbar
}

King Faisal specialist Hospital, Riyadh, SA

Background/Aim Several clinical trials are investigating the use of immune-targeting therapy with Programmed Death Ligand Inhibitors for colorectal cancer (CRC), with promising results for patients with MSI or metastatic CRC. However, the prognostic significance of PD$\mathrm{L} 1$ expression in CRC is controversial and such data is lacking in CRC from Middle Eastern ethnicity. Methods We carried out this large retrospective study to investigate the prognostic and clinicopathological impact of PD-L1 expression in Middle Eastern CRC using immunohistochemistry. A total of 1148 CRC were analyzed for PD-L1 expression. Results High PD-L1 expression was noted in $37.3 \%$ (428/1148) cases and correlated with aggressive features such as high-grade $(p<0.0001)$, larger tumor size $(p=0.0007)$ and mucinous histology $(p=0.0005)$. Interestingly, PD-L1 expression was significantly higher in patients exhibiting high microsatellite instability $(p=0.0169)$. Furthermore, the expression of PD-L1 was found to be an independent marker for overall survival (HR 1.42; 95\% CI 1.03-3.39; $\mathrm{p}=0.0314)$. Conclusion The results of this study indicate that PD-L1 expression can be a valid biomarker for poor prognosis in Middle Eastern CRC patients. This information could help in decision for anti-PD-L1 therapy in Middle Eastern CRC, especially for patients with MSI-H disease.

\section{PREVENTING IATROGENIC URETERIC INJURY DURING LAPAROSCOPIC SIGMOID MOBILISATION: A TRAINEE FRIENDLY APPROACH}

\section{Cho Ee Ng, Aswad Khan, Sharon Ka Po Tam, Ariffullah Khan}

\section{Pulau Pinang, $M Y$}

Background/Aim Colorectal trainees are expected to be competent in laparoscopic colorectal surgeries. However, there is no standardised approach to prevent iatrogenic ureteric injuries during training. We aim to describe a reproducible technique to identify and maintain visualisation of the left ureter during medial to lateral mobilization of the sigmoid colon. Methods Initially, mobilisation of the sigmoid mesentery is performed in the standard fashion. Once the avascular plane of loose areolar tissue is identified, dissection continues along Toldt's fascia with the left ureter confirmed in the retroperitoneal plane. In a patient with fat-filled retroperitoneal space, a separate 
plane of dissection through Toldt's fascia may be developed to clearly identify the left ureter. Once the left ureter is identified, atraumatic dissection to separate it from the surrounding areolar tissue is achieved by gentle stroking of the surrounding loose tissues using 2 blunt tip instruments kept at $1 \mathrm{~cm}$ distances. Following identification and isolation of the left ureter, a rubber vascular loop of $10 \mathrm{~cm}$ is introduced and loop around the ureter and 2 ends of the loop secured using ligaclips. In cases with a duplicated ureter, the number of ligaclips and colour of the loop can help mark each ureter individually. Results Once the loop is placed around the ureter further instrumentation of the ureter can be avoided by grasping the rubber loops. The loop can be positioned inferiorly on the left side of the screen as dissection progresses inferiorly towards the rectum. However, this technique assumes that if the loop's position is in the perceived retroperitoneal position, the left ureter remains away from further dissection. This technique may also help surgeons achieve optimal mesocolic dissection to enable an intact lymphovascular package which has been theorized to reduce tumour cell spillage and possible local recurrence. Conclusion Training in laparoscopic colorectal surgery requires the development of necessary skills in laparoscopy which include differences in visual perception. Aids to help identify important structures should be considered to help training surgeons gain more confidence especially during early training.

\section{MINIMALLY INVASIVE COLORECTAL SURGERY WITHOUT EXTRACTION SCARS: BEYOND STANDARD LAPAROSCOPY}

\section{Bachir Elias, Carine El-hajj \\ Centre hospitalier universitaire Notre Dame des Secours Hospital, Byblos, $L B$}

Background/Aim In an era where laparoscopy is invading colorectal surgery, leaving large parietal extraction scars may reduce patient satisfaction and lead to unwanted postoperative complications. The aim of this paper is to describe and evaluate a minimally invasive surgical technique beyond the standard laparoscopy with a total transanal extraction of the specimen after a laparoscopic sigmoidectomy/left colectomy. We present our series of 22 patients operated with this technique and a Case-video is available. Methods 22 patients benefited from the same technique over 5 years. 15 females and 7 males between 25- and 70-years old Indications were an endoscopically unresectable nonmalignant polyps in the sigmoid (3 patients), diverticular disease (14 patients), or chronic constipation with dolichocolon and or volvulus (5 patients). They all received the same GI preparation to obtain a well clean colon preoperatively. 4 ports were used in every case: an ombilical $10 \mathrm{~mm}$ trocart, two $5 \mathrm{~mm}$ trocarts in the left and right flanc area, and a $12 \mathrm{~mm}$ right iliac region.The sigmoid was dissected without a large lymph nodes dissection. The dissection starts distally at the level of the rectosigmoid junction to isolate the colon at that level.The upper rectum is opened and the anvil of the EEA device is introduced and ligated at the proximal transected end of the colon.The mesocolon is dissected and the resected specimen is extracted through the anus. Then the upper rectum is closed par a Endo GIA and the operation is completed by a termino-terminal transanal colorectal circular anastomosis. Results 21 patients over 22 had laparoscopic colectomy with just four small scars between 5 and $12 \mathrm{~mm}$. Median hospital stay was $48 \mathrm{~h}$ (between $24 \mathrm{~h}$ and 4 days). Due to a very large and thick meso-sigmoid we had one conversion to the standard extraction technique. So far no fistulas, hemorrhages or post-operative abscesses formation where noticed in our review. Patients satisfaction was rated 8 to 9 over 10 concerning esthetic result and postoperative pain. No complications were seen after 3 years follow-up. Conclusion We highly recommend to consider this technique during management of left colic benign pathologies to reduce postoperative abdominal hernias, scarring, infections and pain. Moreover, it will increase patient satisfaction with a better esthetic result. A comparative study with standard left colon laparoscopic surgery can be done to evaluate better this technique.

\section{LIVING WITH A STOMA-THE CORRELATION BETWEEN FUNCTION, PERSONALITY AND ACCEPTANCE}

\author{
Sofia Sandberg, Dan Asplund, David Bock, Carolina Ehrencrona, \\ Björn Ohlsson, Jennifer Park, Jacob Rosenberg, Kenneth Smedh, \\ Sofie Walming, Eva Angenete
}

Sahlgrenska University Hospital/Östra, Göteborg, SE

Background/Aim The gold standard treatment for rectal cancer is surgical resection. If a primary anastomosis is inappropriate, Hartmann's procedure is often performed and if the tumour engages the sphincter, an abdominoperineal excision is required. Both procedures result in a permanent stoma. The patient's acceptance of a stoma is dependent on both clinical and personal factors. Our aim was to characterize stoma function among patients operated for rectal cancer and to investigate if there were any individual factors that had a predictive impact on "bother" related to the stoma 1 and 2 years after diagnosis. Methods The QoLiRECT (Quality of Life in RECTal cancer) study is a prospective multicentre study including 1248 patients with rectal cancer; 472 operated with a permanent stoma. Data from diagnosis and 1- and 2-year follow-up were included. Logistic regression models were used to predict the 1 and 2 year bother using baseline data with the least absolute shrinkage and selection operator (LASSO) to improve prediction accuracy and to address variable importance. Results Number of included patients (returned questionnaire) were $379(80 \%)$. Moderate/major bother was reported by $23 \%$ at 1 year and $17 \%$ at 2 years. Loud flatulence was the most dominant symptom among patients with no/minor bother. Patients with moderate/major bother had an equal prevalence of loud flatulence but also a high proportion of other symptoms (foul-smelling flatulence, leakage, fear of leakage, skin irritation and management difficulties). Fear of leakage was prominent among patients with bother; $39 \%$ reported fear of leakage at least once per day at 1 year (33\% at 2 years). Symptoms were persistent over time. For both 1 and 2 year predictions, level of physical health and quality of life were most important in predicting improved stoma acceptance. Additional important factors were male sex and reduced BMI at 1 year, and a higher rating on the Sense of Coherence scale and increased age at 2 years. The area under the ROC curve (ROC AUC) were 0.40 for both years yielding a sensitivity and specificity of approximately 0.6 . Conclusion A majority of patients were not bothered by their stoma. Bother was related to stoma function. Both physical and mental factors predicted stoma bother at one and two years. However, bother is a multi-factorial problem and accordingly the prediction model is associated with uncertainty. 


\section{A STRUCTURED PRE-SURGERY PRE- HABILITATION FOR ELDERLY PATIENTS UNDERGOING ELECTIVE SURGERY SIGNIFICANTLY IMPROVES SURGICAL OUTCOMES AND REDUCES COST}

\author{
Jingting Wu, Frederick Koh, Caroline Loh, Winson Tan, Leonard Ho, \\ Dulcena Yen, Sharmini Su A Sivarajah, Fung Joon Foo, Min Hoe \\ Chew \\ Sengkang General Hospital, Singapore, SG
}

Background/Aim With increasing global life expectancy, the number of major surgeries done for the elderly invariably increases. As age is a non-modifiable risk factor for increased morbidity and mortality, it is therefore important for peri-operative optimization of modifiable risk factors in this patient population. The aim of this study is to examine the effectiveness of a structured pre-habilitative programme for elderly colorectal cancer patients in improving surgical outcomes. Methods An interventional cohort study was performed with a retrospectively collected control group. A philanthropically sponsored Programme for Enhanced Elderly Recovery @ SKH (PEERS) was initiated in February 2017 for patients 70 years old and older who were due to undergo major colectomies. The surgical outcomes were compared with that of patients from a similar age group before PEERS was introduced (non-PEERS). These patients were put through a 1-2 month-long programme before surgery which included a geriatric assessment, nutritional supplementation and resistance training. Results Fifty-eight patients, with a median age of 78.5 (70-93) years old, were recruited from a single tertiary institution to undergo PEERS. Baseline characteristics between the groups were similar. There was no significant improvement of anthropometric and functional characteristics before and after PEERS programme. The duration of hospitalization was shorter in the PEERS group (9 vs 11 days, $\mathrm{p}=0.01$ ). Both groups had similar 30 -days overall morbidity and mortality rates. The PEERS group had significant improvement in their median EQ-5D score from 0.70 before surgery to 0.80 at 6 -months post-surgery $(\mathrm{p}=0.01)$. After multivariate analysis, the PEERS group had 6.8 days less $(p=0.018$, CI 1.2-12.4) hospitalization duration after adjusting for type of surgery and complication, this represented an average cost saving of USD $\$ 11,838.80$. Conclusion With a standardized pre-habilitation programme for elderly patients before elective colectomies, duration of hospitalization could be reduced with an improvement in quality of life after surgery. These translated to a reduction in healthcare costs for elderly patients.

\section{DOES RECTAL CANCER TUMORAL VOLUME PREDICT SURGICAL OUTCOME?}

\author{
Tommaso Stecca, Alain Fiorot, Anna Pozza, Marco Scarpa, Silvia \\ Venturini, Imerio Angriman, Cesare Ruffolo, Marco Massani
}

AULSS 2 Marca Trevigiana, Treviso, IT

Background/Aim Magnetic Resonance Imaging (MRI) plays a pivotal role in the management of rectal cancer patients. MRI-based tumoral volumetry has been demonstrated to be a prognostic factor for neoadjuvant chemoradiotherapy response. The aim of our study is to retrospectively evaluate this technique in a cohort of surgical patients. Methods This is a monocentric retrospective study based on a continuous series of patients with histological diagnosis of rectal adenocarcinoma, who underwent surgery with total mesorectal excision, after MRI staging, between January 2012 and April 2017. T2- weighed turbo spin-echo MRI sequences were used to create a handfree region of interest on each slice to calculate tumoral volume at diagnosis and after neoadjuvant treatment. Tumoral Volume Reduction Rate (TVRR) calculated as [(Volume at diagnosis - Volume after neoadjuvant treatment)/Volume at diagnosis $] \times 100$. Statistical analysis performed with MedCalc 17.6 software (MedCalc Software bvba, Belgium). Results 45 patients $(\mathrm{M} / \mathrm{F}=29 / 16)$ affected by a low $(\mathrm{n}=20)$ or high $(\mathrm{n}=25)$ rectal cancer were included. Clinical stage was T1 $(\mathrm{n}=1)$; T2 $(\mathrm{n}=19) ; \mathrm{T} 3(\mathrm{n}=23) ; \mathrm{T} 4(\mathrm{n}=2) ; \mathrm{N}+25 / 45.22$ patients underwent early surgery (Volume $9.96 \pm 10.7$ ); 6 short course radiotherapy (SC-RT) (Volume $19.48 \pm 19.8$ ) and 17 long course chemoradiotherapy (LC-CRT) (Volume $52.78 \pm 54$ ). Tumoral load in early surgical patients was lower than that of LC-CRT ones $(\mathrm{p}=0.003)$. Tumoral Volume after LC-CRT was $18.1 \pm 21.9 \mathrm{~cm} 3$ and mean TVRR was $66 \pm 15.3 \%$ (range 34-85.3\%, p < 0.001). Tumoral volume at diagnosis in all patients showed a significant correlation with the pathological stage of the disease (stage 0 -I versus II-III, $\mathrm{p}=0.03$ ). Survival analysis highlighted that patients with a tumoral volume at diagnosis $\geq 30 \mathrm{~cm}^{3}$ had a lower disease-free survival (DFS) than patients with lower volume $(\mathrm{p}=0.04)$. The DFS curves on the basis of the extent of tumor volume reduction after neoadjuvant CRT (TVRR, cut off $75 \%$ ) showed a divergence in the absence of statistical significance $(p=0.07)$. No correlation was found between tumor volume after neoadjuvant CRT and probability of disease recurrence. Conclusion Nowadays, in the "watch \& wait" philosophy, an additional method such as TVRR that can allow the selection of patients with a lower risk of recurrence and with a higher probability of complete pathological response after neoadjuvant CRT could improve the management of patients with locally advanced rectal cancer.

\section{RARE PRESENTATION OF SIGMOID ADENOCARCINOMA AS STRANGULATED FULL THICKNESS SIGMOIDO-RECTAL PROLAPSE}

\section{Stefano Lafranceschina. Marialessia Milella, Anna Stella Lippolis, Vito Dagostino}

Ospedale San Paolo, Bari, IT

Background/Aim Colorectal cancer is one of the most common neoplasm involved in colorectal intussusception in adults. Prolapse is demonstrated to be intussusception of the rectal wall starting $6-8 \mathrm{~cm}$ from the anal verge. It occurs mainly in old women due to laxity of pelvic floor musculature and weakness of the anal sphincters. We present surgical management of a rare case of sigmoid adenocarcinoma presenting as strangulated full thickness sigmoido-rectal prolapse in an old Caucasian woman. Methods A 80 years old Caucasian woman presented with a 4-month history of rectal bleeding and recent presentation of prolapsed sigmoid colon. Her past medical history was significant for constipation and atrial fibrillation. On admission, abdominal examination revealed distension, diffuse tenderness, and few metallic bowel sounds. Rectal examination revealed initial signs of strangulated prolapsed sigmoid colon with evident neoplasm on the prolapsed mucosal surface. A biopsy was performed before surgery, revealing the presence of Adenocarcinoma. CT scan showed a $58 \times 35 \mathrm{~mm}$ neoplasm causing colonic stenosis without secondary localization. Oncological left colectomy with latero-terminal colo-rectal anastomosis with circular stapler was performed, after hand-reduction of the intususcepted colon in surgical theatre. Results On the tithe post-operative day, due to an episode of vomiting, a CT scan was performed. It showed ileus resolved in two days. She was discharged on 17 th post-operative day. Histopatological exam confirmed the presence in the prolapsed colon of an 
adenocarcinoma (pT3N1M0R0). Conclusion Colon cancer associated with sigmoid colon prolapse is a rare condition and colorectal tumors are not usually mentioned as a cause of prolapse. It is important to take in mind that tumors above the rectum may also lead to rectal prolapse due to intussusception of the tumor: a patient with a sudden appearance of voluminous prolapse should be investigated for a colonic tumor by CT scan, colonoscopy and biopsy. This kind of management in order to obtain an early diagnosis and perform the correct surgical treatment for oncological purposes.

\section{FIT TESTING-A KEY TO PRIORITISING URGENT COLONOSCOPIES DURING THE PANDEMIC}

Anuradha Ashok Thrikandiyur, Sreelakshmi Suresh, Rajat Raghunath, Subash Vasudevan ESNEFT, Colchester, Essex, GB

Background/Aim To determine whether the FIT test can be used as a prioritisation tool for patients referred on the Colorectal Cancer pathway. Methods A retrospective study of all patients referred on the 2-week wait, Colorectal Cancer Target Pathway between March 2020 and July 2020 (first peak of COVID in the UK). A detailed analysis was performed identifying the referring symptoms, blood investigations, the FIT results and the results of the colonoscopies. Results A total of 579 patients were analysed, with equal predisposition among both genders. 39 patients $(6.7 \%)$ had a positive diagnosis for Colorectal Cancer. Due to non-reliability of FIT in patients with PR bleed, these patients were excluded from the study and a total of 334 patients were analysed. 284 patients underwent the FIT test while on the Target pathway, with a return rate of $82 \%$ (234 of 284). 94 patients (33\%) had a FIT result of $>10 \mu \mathrm{g}$. Amongst them, 60 were positive for either cancer or significant bowel pathologies. Around $12 \%$ of patients had a false positive result.A total of 15 patients had a positive diagnosis for Colorectal Cancer. Amongst them, 12 patients had a FIT test in the community, of which 11 had a FIT of $>10 \mu \mathrm{g}$. Only 1 patient had a FIT of $<10 \mu \mathrm{g}$, referred on the pathway with Iron Deficiency Anaemia. All these patients were noted to have had urgent prioritisation of their colonoscopies on the target pathway. Amongst the patients diagnosed with Colorectal Cancer, 8 patients $(66 \%)$ had a FIT result of $>100$ $\mu \mathrm{g} .3$ patients $(25 \%)$ had FIT in the range of $10-100 \mu \mathrm{g}$, referred on the pathway with the primary symptom of Iron Deficiency Anaemia. Conclusion With careful interpretation of FIT values, there is potential for risk stratification of patients with prioritisation of investigations on the Target Pathway. Our study lends support to existing evidence, to show that strongly positive results indicate high rates of detection of cancers, however lower to normal values are seen in patients with Iron Deficiency Anaemia. Thus, correlation with clinical history and symptomatology is important, to avoid missing early diagnoses.

\section{INITIATION OF A FAECAL IMMUNOCHEMICAL TESTING (FIT) SERVICE FOR PATIENTS WITH SUSPECTED COLORECTAL CANCER IN THE COVID-19 PANDEMIC}

\section{Adam O'Connor, Victoria Lennon, Naomi Mackenzie \\ Wrightington Wigan and Leigh NHS trust, Salford Quays, GB}

Background/Aim Faecal immunochemical testing (FIT) potentially has a role in the triage of suspected colorectal cancer patients and are recommended by the National Institute for Health and Care Excellence to guide referral of patients with low-risk bowel symptoms in the UK. We describe a short case series of our own new local approach to commencing FIT as part of colorectal cancer referrals from local general practitioners (GP), taking into account the challenges of the COVID-19 pandemic. Methods Patients referred under the colorectal cancer pathway in the Wigan area with symptoms of altered bowel habit $(\mathrm{ABH})$, weight loss, abdominal pain or non-iron deficiency anaemia, were triaged to FIT clinic. This involved a colorectal nurse specialist phoning the patient and arranging FIT testing. In cases with weight loss, CT scan is arranged. If FIT is negative, the patient attends virtual clinic with a surgeon where step-down or further investigation occurs. If FIT is positive, the nurse specialist arranges either colonoscopy or CT colonography based on patient fitness. We recorded data for positive and negative FIT numbers, cancer diagnosis, investigations performed and pathology encountered (for positive patients), across 3 months from June to August 2020. Results Median age of patients was 69 years-of-age (36-90). 13 patients had positive FIT results and 47 negative. Of the positives, 8 had colonoscopy, 5 CT. 1 positive patient had a diagnosis of colorectal cancer and was sent for surgery. The rest of pathologies were split evenly between adenoma and diverticular disease. Of the negatives, 2 patients had underlying malignancy on CT scan however these were not colorectal cancers. 25 had colonoscopy, 20 had CT and 2 had no investigations, as their symptoms had resolved at virtual clinic and they were deemed low-risk for any pathology. Conclusion FIT testing may have a role to play in triaging low-risk colorectal cancer referrals, especially in those patients with negative results. We describe a safe way in the COVID-19 era to establish a FIT testing service in a local district general hospital lacking the resources of a tertiary centre. Larger studies are ongoing regarding the wider application of FIT and locally discussions continue with our cancer centre colleagues regarding safe step down from the cancer pathway.

\section{LONGO VERSUS MILLIGAN-MORGAN, WHAT TO CHOOSE?}

Rita Pereira, Inês Sousa, Miguel Neves, Paulo Alves, Nuno Rama, Vitor Faria

\section{Centro Hospitalar Barreiro Montijo, Barreiro, PT}

Background/Aim Hemorrhoidal disease has a high incidence and prevalence. There are several therapeutic options, however surgery is the only curative method. Surgical treatment has evolved in recent years. The aim of this study was to analyze the surgical approach to hemorrhoidal disease in our center. Methods Retrospective analysis of patients that underwent surgical treatment for hemorrhoidal disease, between March 2008 and October 2018. Results A total of 191 patients were included, $55 \%$ male, with a mean age of 51 years. Most patients had grade III and IV hemorrhoids. 167 (87\%) underwent elective surgery and $24(13 \%)$ urgent surgery. The most used technique was the stapled Longo procedure $(70.6 \%$ versus $23.6 \%$ Milligan-Morgan hemorrhoidectomy). The mean length of stay was 1.04 days. In $17 \%$ of patients we found early complications, the most frequent being pain and bleeding with no statistically significant difference between the two techniques $(p=0.9)$. Late complications were also found in $12 \%$ of the patients, especially pain, anal fissures, bleeding and fecal incontinence, also without statistically significant difference between both techniques $(\mathrm{p}=0.2)$. The recurrence rate was also assessed. This occurred in $14 \%$ of the patients, $9 \%$ needed surgical re-intervention. There was no statistical significance when comparing the rate of recurrence between stapled Longo procedure end Milligan-Morgan hemorrhoidectomy $(p=0.9)$. Conclusion 
There are several options for the surgical treatment of hemorrhoids, the most used and validated techniques are Milligan-Morgan hemorrhoidectomy and the stapled Longo procedure. Pain and bleeding are the most frequent complications. In this study we found similar rates of complications and recurrence with both techniques.

\section{SURGICAL TREATMENT OF PILONIDAL SINUS IN CHILDREN: WHICH METHOD?}

Can Ihsan öztorun ${ }^{1}$, Gökhan demirtas ${ }^{2}$, Elif Emel Erten ${ }^{2}$, Sabri Demir $^{2}$, Hayal Doruk ${ }^{2}$, Ahmet Ertürk ${ }^{2}$, Dogus Güney ${ }^{1}$, Müjdem Nur Azili $^{1}$, Hüseyin Tugrul Tiryaki ${ }^{3}$, Emrah Senel ${ }^{1}$

${ }^{1}$ Ankara Yildirim Beyazit University, PediatricSurgeryClinic, ${ }^{2}$ Ankara City Hospital, PediatricSurgeryClinic, ${ }^{3}$ HealthScienceUniversity, AnkaraCity Hospital, PediatricSurgeryClinic

Background/Aim Ideal approach in pilonoidal sinus treatment; to eliminate the sinus canal, prevent recurrence, have low hospitalization rates, low cost effectiveness and low morbidity rates. In this study, our aim is to retrospectively examine two different surgical methods used in the treatment of pilonidal sinus in our clinic and compare them in terms of complications and recurrence. Methods 122 patients who were diagnosed with sacrococcygeal pilonidal sinus and operated between January 2010 and January 2019 in our clinic were included in our study. Ethical approval was obtained from our hospital for the study. The files and computer records of the patients were retrospectively analyzed in terms of age, gender, body mass index (BMI), applied surgical method, post-op infection, post-op bleeding, recurrence, post-recurrence treatment and the time between the surgeries. The two surgical methods applied in treatment were compared in terms of postoperative complications and recurrence. Results In the study, $62(50.8 \%)$ patients are treated with primary repair method and $60(49.2 \%)$ patients are treated with the limberg flap method. The number of boys/girls of the cases was 73/49. Their average age was 15.4 (2-18) years. Body mass index (BMI) of the cases was 26.90 (18-40.62). Antibiotherapy was given to $94 \%$ of the cases before the operation. In the post-op period of primer repair group, local infection was seen in 12 patients, bleeding and granulation in 7 patients and recurrence in 8 patients. In limberg flap group, local infection was seen in 6 patients, bleeding and granulation in 3 patients and recurrence in 2 patients. In patients with Limberg flap, it was found to be lower in terms of post-op complications. $(\mathrm{P}=0.026)$ Recurrence rate was $8.1 \%$ in girls and $8.2 \%$ in boys. There was no significant difference between post-op complications and gender. It was statistically shown that the BMI was higher in patients with recurrence $(\mathrm{P}=0.03)$. Primary repair was applied to five of the patients with recurrence and flap method was applied to five of them. The mean time between the first surgery and the second surgery was 8.48 months (2-26 months). Conclusion There is no consensus yet on an ideal treatment method for pilonidal sinüs disease in children. Based on this study, we recommend that performing the Limberg flap technique because it has low complication and recurrence rates.

\section{LAPAROSCOPIC ABSCESS DRAINAGE IN PATIENTS PRESENTING WITH ACUTE DIVERTICULITIS WITH PURULENT PERITONITIS: A CASE SERIES}

\author{
Erika Haberfellner Windsor, Elise Quint, Vladislav Khokhotva
}

Windosor, ON, CA

Background/Aim Hinchey III diverticulitis management remains challenging. Hartmann's procedure is associated with significant morbidity and mortality and is usually performed by open approach. "Laparoscopic lavage" has been shown to be associated with increased rates of re-operation and post-operative sepsis. Few studies have investigated the role of laparoscopic abscess drainage without lavage for Hinchey III diverticulitis. Methods We performed a retrospective chart review of patients treated for acute diverticulitis at our surgical center in Ontario, Canada. From 2017 to 2019, 8 patients with Hinchey III diverticulitis had laparoscopic abscess drainage. Patients, who were initially treated with IV antibiotics, but did not respond had a repeat $\mathrm{CT}$ scan. Those who had abscesses inaccessible for percutaneous drainage or progression of un-contained perforation were taken to surgery. Laparoscopy was performed. Where the perforation was minimal or had sealed, abdominal cavity was explored and all abscesses were suctioned. Drains were left in place. IV antibiotics were continued post-operatively. Patients were discharged home with drain in situ. Laparoscopic sigmoid colectomy was offered if there was ongoing chronic drainage or symptoms of recurrent diverticulitis. Results $75 \%$ were male patients, ages range from 48 to 64. Four patients did not require any further surgical treatment during a mean follow-up of 18 months (12-22 months). Four patients underwent laparoscopic sigmoid colectomy on elective basis at an interval of 3.5 weeks to 3.5 months. Unlike the results reported with "laparoscopic lavage", zero patients required urgent re-operation. In all instances elective sigmoid colectomy was completed laparoscopically. Conclusion Laparoscopic drainage of abscesses can be a useful technique as a definitive management of Hinchey III diverticulitis. Where there will be a need for a definitive sigmoid resection at a later time, laparoscopic abscess drainage can facilitate definitive management without a laparotomy or colostomy. Our results indicate that laparoscopic drainage without lavage may be a good management strategy for selected patients presenting with Hinchey III diverticulitis.

\section{READMISSION INDICATED BY SEVERE FLUID- ELECTROLYTE DISTURBANCES AS A COMPLICATION OF ILEOSTOMY}

\author{
Kornél Vajda, Ildiko Horti, Kornélia Trepák, László Sikorszki \\ Kecskemét
}

Sebészet haladása-kisebb kockázatú mtétek alapitványa, Adószám: 19047269-1-03, Kecskemét, HU

Background/Aim After the operations of rectal tumors which were preceded by neoadjuvant chemo-radio therapy, it has become a common practice to create a defunctioning ostomy in order to prevent complications due to anastomotic leak. The ileostomy itself can be the source of complications with $20-60 \%$ incidence rate, while dehydration causes $16,9-40 \%$ of readmissions. Aims: Our goal was to review our own cases on the basis of complications of ileostomy particurarly with regard to dehydration and its consequences. We wanted to develop a therapeutic protocol to help prevent these severe complications. Methods We retrospectively analyzed the morbidity 
data of our patients who were operated on defunctining ileostomy because of different indications between 01.09.2017 and 31.12.2019. During this period, 252 resections of rectum and 33 abdominoperineal resections of rectum were performed. Results Ileostomy was created for 110 patients. 27 patients $(24.5 \%)$ were readmitted with severe renal impairment and electrolyte disturbances. 24 patients were readmitted once, 1 patient twice and 2 patients 3 times. Readmission occurred an average of 49.6 days (1-343) after the operation. At admission and readmission the average of GFR(glomerular filtration rate) of patients was $54.66(38-60) \mathrm{ml} / \mathrm{min} / 1.73 \mathrm{~m}^{2}-22.8(5-51) \mathrm{ml} /$ $\min / 1.73 \mathrm{~m}^{2}(\mathrm{p}=0.001)$, the average of serum $\mathrm{Na}$ (sodium) 140.7 (133-145) $\mathrm{mmol} / \mathrm{l}-1.4(111-144) \mathrm{mmol} / \mathrm{l}(\mathrm{p}=0.001)$, the average of serumK (potassium) 4.6(3.2-5.6) $\mathrm{mmol} / \mathrm{l}-5.37$ (3.6-7.6) $\mathrm{mmol} / \mathrm{l}$ $(\mathrm{p}=0.005)$ and the average of serum creatinine $89.6(54-149) \mu \mathrm{mol} /$ 1-33.3 (107-877) $\mu \mathrm{mol} / 1(\mathrm{p}=0.001)$. Conclusion With regard to the short and long term severe complications of dehydration and the high risk of readmission caused by ileostomy, it is important to estimate the risk of patients, to educate the inpatients as early as possible, to commence the prevention of dehydration, and regularly monitor the outpatient setting. Provided the conditions are given, the early closure of ileostomy should be considered.

The kinetic of white cell count between the first and the third postoperative day is the best parameter to predict anastomotic leaks and surgical site infection after colorectal surgery. A prospective bicentric uncontrolled trial.

Mahdi Bouassida, Ghazi Laamiri, Mohamed Ben Mabrouk, Slim Zribi, Helmi Slama, Bassem Mroua, Mohamed Mongi Mighri, Ali Ben Ali, Hassen Touinsi

\section{Mohamed Tahar Maamouri Hospital, Tunisia, TN}

Background/Aim The objective of this study was to determine the ability of biomarkers: $\mathrm{C}$ reactive protein $(\mathrm{CRP})$ at the 3 rd postoperative day (POD3), white cell count (WBC) at POD3, CRP (CRP at POD3-CRP at POD1) and WBC (WBC at POD3-WBC at POD1), to predict anastomotic leak (AL) and surgical site infection (SSI) after colorectal surgery. Methods This was a prospective bicentric uncontrolled trial. The ability of each parameter to predict AL or SSI was attested by 2 ways: -Area under the curve (AUC) was determined using receiver operating characteristic (ROC) curves analysis which allows determining the optimal cut-off point with the best sensitivity and specificity of each factor to predict AL and SSI. -Univariate and multivariate analysis to determine the weight of each variable in predicting AL and SSI. Results During 1 year study period, 175 patients were enrolled. 29 patients had AL (16.6\%). 55 patients had SSI (31.4\%). The optimum cutoff for WBC to predict AL using the ROC analysis was $2890 / \mathrm{mm}^{3}$. The sensitivity and specificity at the cutoff point were 89.7 and $88.4 \%$, respectively. The AUC at the cutoff point was 0.95 . WBC had the best AUC, the best sensitivity and specificity to predict AL when compared to the other parameters. Three independent predictive factors significantly associated with $\mathrm{AL}$ were identified in multivariate analysis: $\mathrm{mFI}$ (The Modified Frailty Index): OR $=2.76, \mathrm{WBC}: \mathrm{OR}=43.90, \mathrm{CRP}:$ OR $=8.24$. The optimum cutoff for WBC to predict SSI using the ROC analysis was $1350 / \mathrm{mm} 3$. The sensitivity and specificity at the cutoff point were 87.00 and $89.10 \%$, respectively. The AUC at the cutoff point was 0.92 . WBC had the best AUC, the best sensitivity and specificity to predict SSI when compared to the other parameters. Five independent predictive factors significantly associated with SSI were identified in multivariate analysis: $\mathrm{mFI}: \mathrm{OR}=2.23$, Diabetes mellitus: $\mathrm{OR}=3.61$, $\mathrm{WBC}$ at POD3: $\mathrm{OR}=6.05, \mathrm{CRP}: \mathrm{OR}=8.33, \mathrm{WBC}: \mathrm{OR}=13.90$. WBC levels $<1150$ had a sensitivity of $100 \%$ and a negative predictive value of $100 \%$ to predict the absence of AL thus allowing safe and early discharge. Conclusion This study showed that WBC was the most accurate parameter for the early detection of AL and SSI after colorectal surgery. First because it had the best discriminative power in the diagnosis of AL and SSI according to the ROC curves (the best AUC). Second because it had the highest Odds ratio in multivariate analysis for the prediction of AL and SSI (43.90 and 13.90 , respectively).

\section{PSEUDOMYXOMA PERITONEI PRESENTING WITH COMPLETE PELVIC ORGAN PROLAPSE}

\section{Shane Belvedere, Mikael Soucisse}

Royal Melbourne Hospital, Parkville, AU

Background/Aim Case report. Methods Case report. Results A 67-year-old female presented with several months of increasing abdominal distension and significant uterine prolapse. Her past medical history included auto-immune connective tissue disease and hysterectomy. Examination identified a grossly distended abdomen and stage 4 pelvic organ prolapse (POP). Mucin was noted at the appendiceal orifice on colonoscopy, with cytology of subsequent ascitic tap demonstrating acellular mucin. Pseudomyxoma peritonei (PMP) was hence diagnosed. Uterine prolapse was demonstrated on PET imaging. She proceeded to cytoreductive surgery (CRS) in the form of an extraperitoneal total parietal peritonectomy, right hemicolectomy, omentectomy, splenectomy, and bilateral oophorectomy, followed by hyperthermic intraperitoneal chemotherapy (HIPEC). Histopathology demonstrated low grade appendiceal mucinous neoplasm without definite evidence of rupture, and widespread low-grade PMP. She had an uncomplicated recovery and was discharged home on post-operative day 11 . Conclusion Pseudomyxoma peritonei is a rare, slowly progressive condition characterised by diffuse mucinous fluid that fills the peritoneal cavity, caused by mucin producing tumours of the appendix. As the appendiceal lesion grows, the orifice is occluded and mucin accumulates until the appendix ruptures, with the peritoneal cavity then seeded by mucin producing cells. Previous treatment entailed repeated procedures to evacuate mucin, with the patient eventually dying due to bowel obstruction. More recently patients are treated with CRS and HIPEC with significantly improved outcomes. The 10-year overall survival of patients treated with CRS and HIPEC averages 70\%. Pelvic organ prolapse is defined as the downward descent of the pelvic organs to or beyond the vaginal walls. POP is a common condition with multiple risk factors including advancing age, obesity and parity. The role of hysterectomy in POP is debated, although studies have demonstrated that hysterectomy is associated with increased incidence of POP. Apart from being diagnosed incidentally at time of appendicectomy, PMP most commonly presents as increasing abdominal girth, new onset hernia, or vague abdominal discomfort. Presentation with pelvic organ prolapse is rare, being reported in only a few cases. This is a rare case of PMP presenting with complete pelvic organ prolapse.

\section{DIAGMMR, A NOVEL CARRIER TEST TO DETECT INHERITED LYNCH SYNDROME}

Minttu Kansikas, Laura Vähätalo, Jaana Putula, Mariann Kasela, Niklas Lahti, Philippe Arnez, Pä̈vi Peltomäki,Toni Seppälä, Laura Renkonen-Sinisalo, Anna Lepistö, Jukka-Pekka Mecklin, Minna Nyström

LS CancerDiag LTD, Helsinki, FI

Background/Aim Lynch syndrome (LS) is a cancer syndrome caused by inherited DNA mismatch repair (MMR) deficiency. It is the most 
common single cause of hereditary colorectal cancer and it estimated to be carried by up to $1: 125$ people. Current methods to detect LS carriers rely on the identification of MMR disrupting germline sequence alterations, often found from individuals who have already had cancers. The problem is that although there are already thousands of genetic changes found in the MMR genes, not all of them are disease-causing. Moreover, cancer causing mutations, especially in non-coding regions, are not easily or even impossible to be detected. Methods The functional DiagMMR test detects inherited repair deficiency of key MMR proteins MSH2 and MSH6, i.e. LS, from a small $2 \mathrm{~mm}$ skin biopsy taken from the arm. The mismatch repair function of the primary fibroblast proteins is tested using an error containing DNA substrate yielding an easily interpreted result demonstrating either MMR proficiency or MMR deficiency (i.e. LS). During the assay validation, the DiagMMR results of 117 samples were compared to the clinical LS diagnosis determined by sequencing. Samples were collected by Helsinki University Hospital and Jyväskylä Central Hospital, Finland and represented a wide range of MSH2 and MSH6 pathogenic variants $(n=27)$ and non-pathogenic controls $(n=90)$. Results The DiagMMR validation demonstrated the assay specificity to be $100 \%$ (no false positives) while the assay sensitivity was $89 \%$ (three MMR proficient samples with a variation). Overall, the reduced ability to repair the mismatched substrate DNA correlated well with the clinical LS diagnosis. Conclusion The DiagMMR method quantifies the DNA repair efficiency. It can be performed on healthy, unaffected tissue and does not require tumor phenotype or genotype derived information. As such, it may be used alone or to complement an array of cancer reactive tests, to reveal the functional significance of an already found alteration (VUS, loss of MMR protein in IHC, MSI). The DiagMMR test has been CE marked for clinical use since 2019.

\section{DIAGNOSING COLORECTAL NETS-SURVEY OF CHALLENGES IN ACCESS TO DIAGNOSTICS AND TREATMENT FOR NEUROENDOCRINE TUMOR (NET) PATIENTS (SCAN)}

\author{
Mark McDonnell, Christine Rodien-Louw, Catherine Bouvier, \\ Teodora Kolarova, Marianne Pavel, Harjit Singh, Jie Chen, James \\ Howe, Simron Singh, Dermot O'Toole, Dirk Van Genechten, Elyse \\ Gellerman, Sugandha Dureja, Simone Leyden
}

\section{NET Patient Network Ireland, Dublin, IE}

Background/Aim Neuroendocrine tumors (NETs) are complex neoplasms with increasing incidence and prevalence worldwide. SCAN assessed global delivery of healthcare to NET patients. This analysis focused on early diagnosis of NET patients with colonic and rectal primary NET. Methods During Sept-Nov 2019, 2359 NET patients and 436 healthcare professionals (HCPs) from 68 countries completed an online self-report survey, available in 14 languages, disseminated via social media and NET patient group networks. Results Globally, $7 \%$ [153/2359] were diagnosed with primary NET of the colon [39\% $(59 / 153)]$ and the rectum [61\% (94/153)], with an approximately even split between Asia [37\% (57/153)] and Europe [39\% (60/153)]. Average patient age at the time of diagnosis was 51 years globally with an average of 4 years living with the disease. No statistical differences were observed by geographic regions or between the two types of primary NETs. Every third colorectal NET patient had stage IV NETs at diagnosis (32\% [49/153]), NET colon patients with Stage IV 51\% (30/58), NET rectum with Stage IV or METs 20\% (19/97). Slightly above a third of NET patients reported they were misdiagnosed at least once with another condition prior to receiving the correct diagnosis (34\% [52/153]). NET was discovered incidentally while testing for another condition in another third of the cases (31\% [48/153]). Only in 31\% of patients colorectal NET was the first diagnosis received after initial symptoms. Mean time to correct diagnosis for the misdiagnosed was 4.16 years $(\mathrm{N}=52)$. The most common misdiagnosed conditions were gastritis (54\% (28/52)] and Irritable Bowel Syndrome (IBS) (50\% (26/52)]. The diagnostic tools that most often led to the correct diagnosis were biopsy (65\% [98/ 153] and colonoscopy (47\% [71/153]). The clinicians s who most commonly suggested the test were gastroenterologists [34\% (54/153)] or surgeons [20\% (30/153)]. The majority of colorectal NET patients received their diagnosis at a hospital without a NET specialist $(41 \%$ [62/153]). Conclusion Incidental and misdiagnosis lead to an extremely high proportion of NET patients diagnosed with stage IV, associated with poorer patient outcomes and remains a global challenge. More knowledge about NETs among colorectal medical specialists is vital to early detection and timely referral for proper testing.

\section{CANCER REGISTRY DATA OF EASTERN SWITZERLAND DOCUMENT SIGNIFICANTLY DECREASED MORTALITY BY COLORECTAL CANCER AND REVEAL A LARGE POTENTIAL OF A SYSTEMATIC SCREENING PROGRAMME}

\section{Christian Herrmann, Rudolf Morant}

Cancer registry of Eastern Switzerland, St.Gallen, $\mathrm{CH}$

Background/Aim We asked whether the combined effects of important progress of surgery and medical oncology and increased opportunistic screening were able to change the survival and mortality of colorectal cancer. Methods We selected patients with invasive cancers of the colorectum (CRC) from the cancer registry of the cantons St.Gallen and both Appenzell (SGA) for the years 1980-2019. Extent of disease was defined by SEER-groups (localized, regional, distant). Survival analysis was performed using Ederer II-relative survival method with the period approach; giving most upto-date survival estimates accounting for ageing effects. Results CRC incidence is continuously increasing in SGA over the last 40 years, with the strongest increase seen in those under 50 years of age (males $+72 \%$, females $+60 \%$ ), followed by those in the screening age group of $50-74(+56 \%$ and $+47 \%)$. In the same time, CRC mortality decreased on average by $44 \%$ for all subgroups. No significant stage shift was observed over time. Relative survival improved considerably over the last decades, with no significant differences among the sexes. For patients with localized disease, the good survival still further improved from 1980 to about 1990-1994 and remained on this high level thereafter. Patients with regional disease strongly benefitted from therapeutic advancements over the last years, allthough progress in survival has also flattened over the last two decades. Survival of patients with distant metastases was very low but has considerably improved. Median survival of patients with metastatic disease dramatically increased from less than 6 months to more than 20 months now. More and more cases are detected in SGA by opportunistic screening. Until 2000-2004 less than 5\% of registered cases were detected through screening. This has risen to over a quarter of cases in the recent years. Conclusion CRC incidence is on the rise, mortality declines and survival has considerably improved, proving the significant impact of screening, surgery and medical oncology. Although at an increasing rate, currently still less than a third of CRCs are detected by opportunistic screening. This suggests a still large potential of a population based, quality-assured cantonal CRC screening programme addressing the whole population including those being less health conscious. 


\section{IMPACT AND OUTCOMES OF POSTOPERATIVE ANAEMIA IN COLORECTAL CANCER PATIENTS: A SYSTEMATIC REVIEW}

\author{
Manish Chowdhary, Aileen Moncur, Yajing Chu, Nader Francis \\ Yeovil District Hospital, Yeovil, GB
}

Background/Aim Preoperative anaemia is common in colorectal cancer patients. Little attention has been given to the prevalence and consequences of postoperative anaemia. The aim of this study was to systematically review the published literature and determine the knowledge of prevalence and impact of postoperative anaemia in colorectal cancer patients. Methods Databases, CINAHL and Medline, via EBSCO Host, were systematically searched to identify suitable articles published between 2004 and 2020. After an initial search, articles were screened and all eligible articles reported on prevalence of postoperative anaemia, clinical and long-term outcomes data in colorectal cancer patients undergoing surgery were included. The Risk of Bias 2.0 tool for the assessment of Randomised Controlled Trials and Risk of Bias in Non-randomised studies 1.0 tool were used for the assessment of bias in the studies selected in our review. Results Six studies, one randomised control trial and five cohort studies were included with a total population size of 1714 . The prevalence of anaemia at discharge of $76.6 \%$ was reported as the primary endpoint in only one study. The rate of Red Blood Cell Transfusion and length of hospital stay were found to be significantly increased in anaemic patients, while postoperative infection rate results were variable. Quality of life scores and overall survival at 5 years were significantly affected among anaemic patients as reported in two papers. Conclusion The available limited evidence on postoperative anaemia indicates its high prevalence with negative impact on clinical and long-term outcomes. Further research is required to standardise the measurement and address the true impact of correcting postoperative anaemia on functional and oncological outcomes.

\section{PERSONALIZED APPROACH TO CANCER PATIENTS OLDER THAN 70 YEARS WITH COMORBID CARDIOVASCULAR PATHOLOGY DURING ROBOT ASSOCIATED/ LAPAROSCOPIC ABDOMINAL CAVITY AND PELVIS SURGICAL INTERVENTIONS}

\section{Arkady Bedzhanyan, Yulia Frolova, Vladimir Nikoda, Olga Dymova}

Petrovsky National Research Center of Surgery, $R U$

Background/Aim To evaluate the risks of progression of concomitant chronic heart failure (CHF) in cancer patients robot associated/ laparoscopic non-cardiac surgical interventions for abdominal cavity and pelvis cancer. Methods From 2018 to 2019 a 30 elderly patients (pts) with verified cancer and comorbid cardiovascular pathology (67\% male) were analyzed before and after robot associated/laparoscopic surgery for abdominal cavity and pelvis cancer. The mean age was 76 years. $14(47 \%)$ pts had Class II due to RCRI PMP score, 12 (40\%)_Class III, 4 (13\%)—Class IV, 9 (30\%) pts had diabetes Mellitus, $28(93 \%)$ pts-hypertension, $11(37 \%)$ pts-atrial fibrillation, $30(100 \%)$ pts-atherosclerosis of the aorta and its branches, 3 (10\%)-obesity. 20 (67\%) pts had I-II FC NYHA (functional class, New York Heart Association), 10 (33\%)\%-III FC. The level of NTproBNP-1641.8 $\pm 47 \mathrm{pg} / \mathrm{ml}, \quad$ cTnI $<0.01 \mathrm{~g} / \mathrm{mL}$, LDL-cholesterol $3.69 \pm 0.4 \mathrm{~m} / 1, \quad$ CKD $53.5 \pm 3.1 \mathrm{ml} / \mathrm{min} / 1.73 \mathrm{~m}^{2}$. ACE-inhibitor/ angiotensin or BRA receptor blockers received $20(71 \%)$ pts, betablockers-22 (73\%) pts, aldosterone antagonists-5 (17\%) pts, diuretics-3 (11\%) pts, warfarin NOAK-11 (39\%) pts, statins 19 $(68 \%)$ pts. According to ECHO, the mean LVEF $43.2 \pm 2.9 \%$, mean SPLA $23.9 \pm 1.88 \mathrm{mmHg}$, diastolic dysfunction also, $16.9 \pm 3.1$. Results 29 pts were discharge from hospital within $13 \pm 2.5$ days after operation with no clinical changes in FC NYHA. Postoperative death was observed in one case due to septic shock. Conclusion We conclude that only personalized approach to cancer patients older than 70 years with comorbid cardiovascular pathology during robot associated/laparoscopic abdominal cavity and pelvis surgical interventions is beneficial. Standard CV therapies, careful weighing of risks, according to stratification scales together with robot associated/ laparoscopic surgery is beneficial in such patients, who have a higher risk of postoperative cardiac problems. The fundamental stages of determining the risks of developing cardiovascular complications in the pre and early postoperative periods, their personalized diagnosis and prevention by specialists of the "cardio-oncological team".

\section{IMPLEMENTATION OF IDEAL FRAMEWORK OF UK TRAINING PROGRAMME FOR TATME}

Nader Francis, Manish Chowdhary, Marta Penna, Neil Mortensen, Roel Hompes, Fiona Carter

Yeovil District Hospital, Yeovil, GB

Background/Aim As international concern grows regarding the reported early oncological outcomes and technical challenges of transanal total mesorectal excision (TaTME), there is a need for a structured and robust quality assurance process to ensure safe introduction and monitoring of a novel surgical technique. The IDEAL framework has been advocated to guide such a process. The aim of this study was the report the application of IDEAL framework in the development and implementation of TaTME training in the United Kingdom. Methods A five-stage outline [Idea, Development, Exploration, Assessment, and Long-term study] was applied to describe the development, delivery and assessment of the TaTME training initiative in the UK. Surveys that incorporated the experience of both learners and more experienced surgeons of TaTME, together with experts in education, initiated the process with concepts and development of the training initiative explored at a centrally co-ordinated pilot training programme. Key components included a cadaver training workshop and a formal proctorship process. Data were recorded on demographics, tumour location, intraoperative, postoperative and histological outcomes. Educational assessment of technical progress was performed using custom-made Global Assessment Scoring (GAS) forms which were completed by both learners and proctors.

Long term outcomes were captured at 24 months. Results Five selected pilot sites were used by 10 colorectal surgeons during the training initiative and 24 cases were proctored in this period in the exploration phase. Median operative time reduced from initial $331 \pm 90$ (195-610) minutes to $283 \pm 62(195-340)$ in the final case. No visceral injuries were reported however there was one conversion to open $(4.2 \%)$. Histological assessment reported as intact mesorectal TME specimens with clear distal margin and no bowel or tumour perforation in all cases. One case had positive circumferential margin (4\%). Assessment of educational outcomes showed GAS score 5 (Independent performance) was achieved by case 5 in most operative steps. Long term follow up showed no evidence of local or regional recurrence but three liver and one lung metastasis at 24 months. Conclusion Dissemination of a new surgical technique within the 
confines of IDEAL framework demonstrates the feasibility and safety of surgical training programme for TaTME at a national level.

\section{DIEULAFOY'S LESION IN THE CECUM: A RARE PRESENTATION}

Ana Cláudia Ribeiro, Susana Costa, Inês Romero, Sılvia da Silva, Rui Reis

Centro Hospitalar do Tâmega e Sousa, Penafil, PT

Background/Aim Acute gastrointestinal bleeding is a frequent medical condition with potential for significant morbidity and mortality. This is caused, mostly, by peptic ulcers and gastroesophageal or duodenal erosions. Dieulafoy's lesion is a rare entity, normally present in the stomach and more rarely in the colon, responsible for $1 \%$ to $5 \%$ of cases of acute gastrointestinal bleeding. The true incidence may be underrated, since most are asymptomatic and difficult to diagnose, despite endoscopic advances. The aim of this poster is to expose a clinical case of a patient with acute gastrointestinal bleeding due to Dieulafoy's lesion in the cecum. Methods Case's presentation: Woman with 85 years old presented with a clinical condition of haematochezia for two days, associated with anaemia and hemodynamic instability needing blood transfusion. The colonoscopy was performed and demonstrated a Dieulafoy's lesion in the cecum with active bleeding. Endoscopic haemostasis with local injection of adrenaline and placement of haemostatic clips on the vessel was successful. The hospitalization occurred with no further complications. Results Discussion: The diagnosis of Dieulafoy's lesion is difficult because it is a rare condition and clinicians barely think about it. Its endoscopic diagnostic and therapeutic approach is the standard method in the event of an acute gastrointestinal bleeding episode, with greater efficiency when combined haemostatic techniques are used. Surgery is necessary in less than $5 \%$ of cases, when bleeding is not treated either by endoscopic or angiographic techniques. Conclusion Dieulafoy's lesion in the colon is a very rare entity, but with the potential of massive haemorrhage with consequent mortality. Its diagnosis is difficult, mainly due to its rarity, especially when this lesion is found in even less frequent locations. It is essential to be aware of the existence of this lesion as a possible cause of gastrointestinal bleeding and to differentiate it from other causes. Advances in endoscopy have increased the rate of diagnosis of these lesions and reduced their mortality.

\section{ROBOTIC VERSUS LAPAROSCOPIC ANTERIOR RESECTION FOR MID-LOW RECTAL CANCERS AFTER NEOADJUVANT CHEMORADIOTHERAPY: MULTIVARIATE ANALYSIS OF RISK FACTORS FOR LONG-TERM SURVIVAL}

\section{Guglielmo Niccolò Piozzi ${ }^{1}$, Seon Hahn Kim ${ }^{2}$ \\ ${ }^{1}$ Milano, Italy, IT, ${ }^{2}$ Seoul, South Korea, KR}

Background/Aim The introduction of robotics in rectal cancer (RC) surgery carried out theoretical advantages following technological improvements, however robotically assisted TME has not yet demonstrated to provide unequivocal benefits over laparoscopic TME. Most of comparative studies failed to show the short- or longterm oncological superiority of the robotic approach. However, it was associated to lower conversion rates and better post-operative genitourinary function. The present study specifically evaluates the role of surgical approach on the long-term oncological outcomes for mid-low RC submitted to neoadjuvant chemoradiotherapy (nCRT) and anterior rectal resection by comparing robotic to laparoscopic approach. Methods Between September 2006 and August 2018, 175 patients who underwent elective minimally invasive anterior rectal resection for mid-low RC (below $10 \mathrm{~cm}$ from the anal verge) with curative intent after nCRT were extracted from a prospectively maintained colorectal database. Stage IV, synchronous and hereditary RC were excluded. A total of 37 laparoscopic and 138 robotic patients were enrolled. Patient-related, operative and pathological factors were assessed and long-term survival was compared between the surgical groups. Results The groups were homogeneous for demographic characteristics and perioperative clinicopathologic outcomes. Median follow-up was 47 months (range 24-65). Distant recurrences were $29.7 \%$ and $8.7 \%$ in the laparoscopic and robotic group, respectively. Overall survival (OS) rates at 1-, 3- and 5-years were 100\%, $85.7 \%$ and $78.9 \%$ for the laparoscopic group and $97.0 \%, 91.2 \%$ and $89.8 \%$ for the robotic group $(\mathrm{p}=0.048)$. Disease free survival (DFS) rates at 1 -, 3- and 5-years were $86.3 \%, 74.3 \%$ and $66.8 \%$ for the laparoscopic group and $97.7 \%, 92.5 \%$ and $87.7 \%$ for the robotic group $(\mathrm{p}=0.001)$. On multivariate analysis, older age $(\mathrm{p}=0.003)$ and $\mathrm{ypN}(1,2$ vs 0 ; $\mathrm{p}=0.016)$ were risk factors for OS, while older age $(\mathrm{p}=0.026)$, ypN $(1,2$ vs $0 ; p=0.004)$ and surgical approach, in favour of robotics (HR $0.376,95 \%$ CI $0.160-0.885, p=0.025$ ), were risk factors for DFS. Conclusion In this cohort, robotic surgery provided better OS and DFS than laparoscopic surgery for mid-low RC patients who received nCRT and anterior rectal resection. The robotic approach was an independent protective factor for DFS on multivariate analysis. This study justifies further trials to explore the oncological superiority of robotic over laparoscopic resection for RC.

\section{NEW FRONTIER IN TEMPORARY ILEOSTOMY. TEMPORARY PERCUTANEOUS ILEOSTOMY IN EXTRAPERITONEAL COLORECTAL ANASTOMOSIS: A RETROSPECTIVE STUDY IN 150 CASES}

\section{Enrico Mariani}

Trevi, IT

Background/Aim Loop ileostomy is a suitable procedure for transitory fecal diversion after colorectal or coloanal anastomosis. We describe here an easy alternative technique for ileostomy construction that does not require reintervention for the closure. Methods In 150 patients undergoing anterior resection of the extraperitoneal rectum with colorectal and/or coloanal anastomosis, percutaneous ileostomy was performed using first a modified jejunostomy tube and now a new specific device called ALPI tube (Anastomotic Leak Prevention Ileostomy). The device positioning was performed in all cases in the preterminal ileum, directly or through the caecum and ileocecal valve; the balloon was then inflated with the aim of completely occlude the ileum lumen. The device was removed, without surgery, between eighth and tenth post-operative day after a radiological contrast enema of the anastomosis. Results Three local complications were registered in cases of direct introduction of the device in the ileum; two cases of enteric fluid filtration through the introduction hole, caused by failed adhesion to peritoneum, that required reintervention (in a case peritoneal washing and traditional loop ileostomy, peritoneal washing and direct suture of the introduction site, in eighth postoperative day, in the second one). The third case was an extended subcutaneous phlegmon that required a laparoscopic reintervention with direct suture of the introduction site and debridement of abdominal wall. Two anastomotic leaks were registered $(1$ colorectal 
and one coloanal anastomosis) that required a traditional loop ileostomy and a prolonged observation of pelvic drain tubes but not pelvic debridement. No complications were registered in cases of transcecal approach. This technique consented in 147 cases to avoid conventional loop ileostomy and relative complications rate, related to stoma confectioning and closure. Conclusion We described here a safe alternative technique to loop ileostomy with few complications related to local filtration of enteric fluid and two anastomotic leaks. This technique was developed with the aim of avoiding conventional loop ileostomy confectioning, closure and related, not negligible,

complications.

\section{MACHINE LEARNING BASED COLORECTAL CANCER DETECTION USING HYPERSPECTRAL IMAGING: A STEP TOWARDS AUTOMATIC OPTICAL BIOPSY}

\author{
Boris Jansen-Winkeln, Hannes Köhler, Ines Gockel, Kathrin Schierle, \\ Marianne Maktabi
}

Universitätsklinikum Leipzig, Leipzig, DE

Background/Aim Until now, the identification of colorectal carcinoma is done visually during colonoscopy or surgery. The pathologist confirmed the margins of the specimen histologically. For the detection of adenomas and carcinomas, systems of artificial intelligence and deep learning are increasingly used with success. In surgery, tumor detection is not of primary importance, but visualization of the safety distance is very important. Hyperspectral imaging (HSI) is often used in medicine for perfusion assessment, here we combined HSI with methods of artificial intelligence for tissue recognition. Methods Between July 2019 and May 2020, 54 patients with colorectal resections for colorectal cancer or an endoscopically non-resectable adenoma were included and 59 pathologies were examined. After resection, the specimen was cut open and the tumor was captured intraluminal by using a hyperspectral camera with a wide spectral range between 500 and $1000 \mathrm{~nm}$. Afterwards, the images were annotated by a pathologist: tumor lesion, tumor surrounding tissue, and healthy mucosa. Classification and visualization based on a neuronal network was performed. Results Based on a 4-layer-perceptron the classification of malignant tissue (cancer and adenoma) achieved a mean sensitivity of $75 \pm 2 \%$ and a mean specificity of $81 \pm 2 \%$ in a leave-one-patient-out-cross validation. Furthermore, the spectral differences were analyzed and significant difference determined between $\mathrm{T}$ classification, neodjuvant therapy, and cancer based on perfusion parameter (e.g. oxygen saturation). Conclusion Hyperspectral imaging can be applied to differentiate between tumor tissue and healthy mucosa. Thus, the detection of tumor tissue can be independent of visual recognition by physicians.

\section{RESECTION LINE DEFINITION BY HYPERSPECTRAL IMAGING IN COLORECTAL RESECTIONS IS SAFER THAN CLINICAL DECISION}

Boris Jansen-Winkeln, Jaqueline Isabelle, Michele Dvorak, Hannes Köhler, Marianne Maktabi, Ines Gockel

Universitätsklinikum Leipzig, Leipzig, DE

Background/Aim Anastomotic leaks are serious complications. One cause may be poor blood perfusion in the bowel. We show the method of defining the ideal transverse line and anastomotic position by intraoperative hyperspectral imaging (HSI) in colorectal resections and comparison with clinical macroscopic evaluation. Methods Hyperspectral imaging by the TIVITA ${ }^{\circledR}$ Tissue System (Diaspective Vision GmbH, Am Salzhaff, Germany) has the property to record light spectra from 500 to $1000 \mathrm{~nm}$ and to provide information about physiologic parameters of the focused tissue area intraoperatively (e.g., tissue oxygenation and perfusion). We compared macroscopic clinical assessment with hyperspectral measurements to define the ideal resection margin in 98 patients undergoing left sided colorectal resection before and after separation of the marginal artery over $5 \mathrm{~min}$; HSI was performed each minute to assess the parameters mentioned above. Results The false color images calculated from the hyperspectral data visualized the margin of perfusion in 93 out of 98 patients precisely. In 17 cases, there was a deviation between the transection line planned by the surgeon and the border line visualized by HSI more than $5 \mathrm{~mm}$ (towards the proximal site). The biggest drop in perfusion took place after $1 \mathrm{~min}$ after devascularization. The borderline was not straight; the width ranges between 10 and $30 \mathrm{~mm}$ in 15 cases. Conclusion Determination of the resection margin by HSI provides the surgeon with an objective decision aid for assessment of the best possible perfusion and consecutive ideal anastomotic area in colorectal surgery.

\section{ENDOSCOPIC APPROACH TO MINIMISING MORBIDITY IN LARGE PERIANAL ABSCESS}

\section{Kushal Mital}

Medicare hospital, Thane, IN

Background/Aim To decrease healing time, prevent fistula formation and minimise pain. Most complex fistula occurs due to inadequate drainage. Endoscopic abscess drainage leaves no cavity behind. Methods Endoscopic drainage of large perianal abscesses and horseshoe abscess using STORZ fistuloscope $3.3 \mathrm{~mm}$. Using Storz, $3.2 \mathrm{~mm}$ fistuloscope, a $3 \mathrm{~mm}$ incision close to anal verge, drain the abscess. Fistuloscope introduced into abscess cavity using saline irrigation. Curette the abscess walls. Then push in $5 \mathrm{ml}$ methylene blue with $1 \mathrm{cc}$ of $\mathrm{H}_{2} \mathrm{O}_{2}$ to look for internal opening. If visulized, then a fistulotomy is undertaken. Keep drainage tube of $16 \mathrm{Fr}$, for postoperatively irrigation for $48 \mathrm{~h}$. Irrigation with normal saline was twice daily for 48 h. Daily dressing done at home. Results 48 patients, ages between 16 and 68 years, predominantly males $2:: 1$. Average wound healing time 12 to 14 days. Seven patients had horseshoe abscess, where abscess post anal space is opened by fistulotomy and cleared by endoscope all procedures healed. No second intervention was done in any case. Average closure time was 14 days, varying from 8 to 21 days. Conclusion Endoscopic drainage of large anal abscess, is a simple way of draining large abscesses, results show a marked decrease in morbidity by way of pain and healing time, and early return to work, compared to traditional incision and drainage.

\section{PUBORECTALIS MUSCLE RUPTURE: DIAGNOSIS AND REPAIR FOR PERINEAL INSTABILITY}

Mariam Al Ketbi, Joan Robert, Jeremy Meyer, Frédéric Ris, Bruno Roche

Hôpitaux Universitaire de Genève (HUG), Geneve, $\mathrm{CH}$

Background/Aim Puborectal muscle PRM rupture usually arises from peripartum perineal trauma and may result in a rectocele or 
rectal prolapse, with or without anal sphincter lesions. Patients with PRM and levator ani rupture may present with incontinence, evacuation disorder, sexual dysfunction or pelvic organ descent. We describe, for the first time, the functional disorders associated with rupture of the PRM and the techniques we use to diagnose and treat this condition. Methods We constituted a prospective cohort study of patients with levator ani and PRM avulsion in the Proctology and Pelvic Floor Unit in the University Hospital Geneva from January 2001 to November 2018. Clinical examination, anoscopy and U/S were performed routinely. Rupture of the levator ani and PRM was diagnosed by clinical exam and U/S. A Wexner incontinence score was completed before and 6 months after surgery. Levator ani and PRM repair was performed using a transvaginal approach. For the rest of the paper the term PRM will include all or part of the levator ani muscle. Results 52 female patients of median age of 57.8 years were included. 31 patients $(59.6 \%)$ suffered anal incontinence, $25(48.1 \%)$ urinary incontinence, 28 (53.9\%) dyschesia, 20 (38.5\%) dyspareunia, $17(32.7 \%)$ colpophonia (audible passage of gas trapped in vagina), and $13(25.0 \%)$ from impaired sensation during sexual intercourse. Deviation of the anus on the opposite side of the lesion was observed in 50 patients $(96.2 \%)$. From these 52 patients, PR ruptures were categorised into right, 43 (82.69\%), left sided, 7 (13.46\%) and bilateral, $2(3.85 \%)$. PRM repair was performed in all patients, with posterior colpomyorraphy in $26(50 \%)$, and sphincteroplasty in 34 patients (63.4\%). 4 patients experienced post-op complications: postop pain $5.77 \%(3 / 52)$, hematoma $1.92 \%$ (1/52), urinary retention $3.85 \%(2 / 52)$, and perineal abscess in $1.92 \%(1 / 52) .41$ patients (78.8\%) had full restoration of normal PRM function (Wexner: 0/20) after surgery, and all patients had an improvement in the Wexner score and in sexual function. Conclusion Diagnosis of levator ani and PR muscle rupture requires a careful history, physical examination and endo-anal and perineal ultrasonography. Surgical repair improved anal continence and sexual function in all patients. This new surgical procedure seems to be well tolerated with good short and long-term results. Early, routine repair is ideal, but more studies with larger patient numbers and longer follow-up are necessary.

\section{SURVIVAL PATTERN \\ AND CLINICOPATHOLOGICAL DATA FROM A SOUTH ASIAN COHORT OF YOUNG COLORECTAL CANCERS TREATED WITH CURATIVE INTENT}

\author{
Kaushika Gunasekare, Supun Godahewa, Pathum Vishwajith, \\ Shashikala Gajasinghe, Madhushanka Ekanayake, Sumudu \\ Kumarage, Pramodh Chandrasinghe
}

\section{University of Kelaniya, Ragama, LK}

Background/Aim Colorectal cancer (CRC) has the fourth highest incidence among cancers in Sri Lanka. Although CRC is considered as a disease of the elderly, currently there is a shift towards an increasing incidence of young cancers globally. Despite the increasing incidence there is a scarcity of data from the South Asian region. This study aims to analyze the clinicopathological features and longterm survival of young CRC from a South Asian cohort of patients. Methods All patients treated for CRC at a tertiary care center from 1997 to 2017 were prospectively followed up. Demographics, tumour characteristics and survival data were recorded. Age less than 45 years at diagnosis was considered as a young cancer. Overall survival among the populations was compared using Kaplan-Mire survival curves. A P value of $<0.05$ was considered significant. Results A total of 113 (16.5\%) young cancers (Mean age 36.35; range 17-45; female $53.1 \%$ ) were operated during the period. Rectum
(60.2\%) was the commonest site followed by the right colon $(24.8 \%)$ and left colonic (15\%) tumors. Adenocarcinomas of moderately differentiated variety $(72.4 \%)$ was the commonest histological type. Seventy percent of cases had locally advanced disease (T3/T4) with $53.2 \%$ having positive nodal status. Of the total $27 \%$ received neoadjuvant treatment and $66.7 \%$ received adjuvant treatment. Young CRC patients had a significantly better overall survival compared to their older counterparts $(\mathrm{P}=0.008)$. Conclusion Young cancers accounts for a significant proportion of the colorectal cancers in this cohort. Over $75 \%$ of the cancers were on the left colon and the majority was locally advanced disease. Overall survival of the young CRCs were better compared to the older population in this cohort.

\section{RESIDENCY TRAINING PROTOCOL IN LAPAROSCOPIC COLECTOMY}

\author{
Filipa Fonseca, André Caiado, Francisco Cabral, João Maciel, Rita \\ Barroca, Manuel Limbert, Nuno Abecasis
}

IPO-Lisboa, Moita, Alvorninha, PT

Background/Aim In the last few decades we have seen a paradigm shift in the surgical approaches, with a progressive predominance of minimally invasive surgery. This transition requires significant modifications in the resident's learning method. Currently, most of the surgeries performed in our colorectal unit are minimally invasive, therefore laparoscopic training is an essential component in the resident's curriculum. Since there are no standardized national guidelines for the curricular objectives to be met through resident education, there is a heterogeneity in the education of future surgeons. This project aims to present our teaching protocol for laparoscopic colectomy. Methods After a bibliographic research about educational methodology and models, a multigenerational group composed of specialists and residents from distinct phases of their residency program created a new teaching protocol for laparoscopic colectomy. This protocol intends to design the most efficient, effective and sustainable training environment. It is goal-oriented and focused on continuous improving of technical and nontechnical competencies. The main purpose is to improve the learning process and surgical performance while maintaining quality standards. This project is currently being implemented in our colorectal unit. Results In this protocol, we use a competency-based and outcome-focused educational approach. It includes an explicit description of the weekly educational activities, the fundamentals that are transversal to any trainee and a list of theoretical and practical competencies expected at each level. In addition, we created schematic summaries of our systematic and structured approaches to laparoscopic colectomies. This protocol also includes video-based reviews, laparoscopic skills training, supervised practice feedback and scheduled assessments of progression in the established milestones. Conclusion Thus, the surgical training becomes more efficient, diminishing the necessary time to prepare the resident to be able to safely perform a laparoscopic colectomy and provide the best possible care to patients. In the future, the impact of this method on the learning curves will be evaluated.

\section{COLORECTAL CANCER IN YOUNG PATIENTS: THE PRIMARY SINGLE-CENTER ANALYSIS}

\author{
Gleb Khrykov, Nikita Burlov, Sergey Savchuk
}

Saint-Petersburg, $R U$ 
Background/Aim To evaluate an epidemiology and short-term treatment results of young colorectal cancer patients on the base of single-center experience. Methods We obtained data for the interval between 2010 and 2019 years. Selection criteria included age 18 to 49 years at diagnosis of primary CRC who underwent surgery. Exclusion criteria were histology no adenocarcinoma, carcinoma of unknown primary, multiple cancers, in situ disease. Parameters included sex, age, symptoms, primary site, TNM stage (using the 7th or 8th editions, depending on the year of diagnosis), grade. The primary aim was analysis baseline parameters and short-term outcome after surgery treatment. Results 77 patients were included. Median age was 45 years $(40 ; 47)$, proportion of female and male was $51 \%$ vs. 49\%. All patients had symptomatic disease (rectal bleeding, abdominal pain and etc.). The most often tumor was found in left side -57 patients $(75 \%)$ : rectum-38\%, rectosigmoid-16\%, sigmoid- $14 \%$. Disease of stage II was in $37 \%$ of cases, locoregional in $23 \%$ and metastatic in $31 \%$. The majority of patients had a G2-tumor (86\%). In 16 cases $(21 \%)$ had family members with cancer (colorectal, gastric, breast). Laparoscopic operation was performed in $26 \%$ patients (7\% of conversion, $48 \%$ of extended operation). Postoperative complications developed in $33 \%$ cases. On Clavien-Dindo classification severe morbidity (Grade 3-5) was $48 \%$ of all complications. Surgical approach did not effect on morbidity rate. 30-days mortality rate was $0 \%$. Conclusion According to the results of our analysis, there's a high number of patients with left-side cancers. Therefore, the biology of this tumor differs from that found in older patients. Near $20 \%$ of patients had a high incidence of cancer history. This may be a risk factor, but further research is needed. Other parameters do not differ from historical ones and significant differences. We plan to include even more patient in the study for a more accurate primary evaluation and to analyze of long-term oncological outcome.

\section{FISTULECTOMY USING THE NEW TECHNIQUE FIXCISION $^{\circledR}$-PRINCIPLES, TECHNIQUE AND SHORT-TERM RESULTS}

\section{Sofia Daniela Reis, Rita Pereira, Catarina Rolo Santos, Sara Patrocínio, Duarte Gil Alves, Constança Azevedo, Hélder Além, Zara Caetano}

\section{Centro Hospitalar Barreiro Montijo, Lisboa, PT}

Background/Aim Perianal fistula is a complex and frequent disease. Despite scientific advances, no treatment or technique has shown absolute superiority in terms of efficacy and recurrence rate. We aim to describe the new existent technique used to treat transsphincteric and intersphincteric fistulas and to present the short-term results of our center. Methods This study was performed in patients who underwent FiXcision technique during January 2020 at our institution. Their medical data were retrieved from the electronic patient files. The fistula characteristics were described by physical examination plus MRI and were classified according to the Parks classification. Recurrence rate, postoperative pain and fecal incontinence were reviewed. Results We performed this technique in ten patients, six women $(60 \%)$ and four men $(40 \%)$ with a mean age of 56.2 years old. All patients present a low level of postoperative pain, short recovery period and early return to work. This procedure did not affect the occurrence of fecal incontinence or soiling. During a median followup of 10 months, we observed complete healing of the fistula tract in $90 \%$ of the patients. There was no significant difference between the healing rates of transsphincteric and intersphincteric fistulas. Conclusion In the authors' experience, the FiXcision technique is easy to learn and can be done in just a few simple steps. So far it has proved to be a good technique for the treatment of simple anal fistula with a high healing rate, minimal invasiveness, quick healing, and without disturbance to anal function.

\section{REGIONAL STUDY PROTOCOL FOR IMPLEMENTATION OF ERAS (ENHANCED RECOVERY AFTER SURGERY) PROTOCOL IN COLORECTAL SURGERY: A STEPPED WEDGE CLUSTER RANDOMISED CONTROLLED TRIAL}

Luca Pellegrino, Eva Pagano, Alessandra Marano, Danilo Donati, Maurizio Meineri, Monica Rolfo, Giovannino Ciccone, Felice Borghi

ASO Santa Croce e Carle di Cuneo, Cuneo, IT

Background/Aim Enhanced recovery after surgery (ERAS) protocol is a multimodal perioperative care pathway designed to achieve an early recovery of patients undergoing major surgical procedures. Even if the protocol, particularly in colorectal surgery, has shown significant advantages in terms of reduction of morbidity, post-operative hospital stay and healthcare costs its diffusion is limited to few selected centres. The aim of this study is to evaluate the results deriving from a top-down implementation of the ERAS protocol in colorectal surgery to a whole regional network of hospitals in the north-west of Italy (Piedmont Region). Methods The study protocol is a multicenter cluster randomized controlled study, with stepped wedge design, comparing ERAS protocol to standard perioperative care. The primary outcome is the length of hospital stay (LOS). Secondary outcomes are: incidence of post-operative complications, time to patients' recovery, control of pain, patients' satisfaction, and healthcare costs. All the surgical departments of Piedmont Region, that perform more than 30 colorectal cancer surgery for years, are involved. All the patients undergoing elective colorectal cancer surgery, both with minimally invasive or laparotomic approach, are recruited during the study period. On the basis of a randomized order, each hospital switches from current clinical practice (control group) to the adoption of the ERAS protocol (ERAS group) every three months. In the quarter preceding the date of randomization, specific training on the ERAS principles is provided to a selected multidisciplinary group. All participants are further required to train their colleagues at local level. The expected sample size of about 2200 patients (1100 in the ERAS group and 1100 in the control group) has a high statistical power (98\%) to detect a reduction of LOS of 1 day and to estimate clinically meaningful changes in the other study endpoints. The study protocol has been approved by the Ethical Committee of the participating centers. Results The enrollment started the 1st September 2019. The end of recruitment, prolonged of three months due to COVID-19 outbreak, is now expected in February 2021. At the end of October 2020, the number of patients enrolled is about 1900. Conclusion Study results will be timely circulated within the hospital network and published in peer-reviewed journals.

\section{MANAGEMENT OF PERIANAL ABSCESS AND FISTULA-IN-ANO IN CHILDREN}

\author{
E. Erten, C. Öztorun, A. Ertürk, S. Demir, R. Kar, D. Güney, M. Azili, \\ E. Senel
}

Ankara City Hospital, Pediatric Surgery Clinic, Ankara Yildirim Beyazit University, Pediatric Surgery Clinic 
Background/Aim The aim of this study is to evaluate the clinical characteristics of perianal abscess and fistula-in-ano in children and to assess our experience in the treatment. Methods A retrospective analysis of the records was performed for the children who received perianal abscess and fistula-in-ano treatment between January 2014 and September 2020. Demographic information of the patients, complaints, treatment procedures, abscess recurrence, and development of fistula-in-ano were evaluted. Patients with systemic diseases and inflammatory bowel diseases were excluded from the study. Results A total of 292 children ( 215 boys, 77 girls) treated for perianal abscess and fistula-in-ano with a median age of 9.3 months (1-216) were included for the study. Patients were divided into two groups according to age distribution, 160 of the patients were younger than 12 months, and 132 were older than 12 months of age. There was no significant difference in sex distribution, treatment procedures, recurrence of abscess and fistula-in-ano formation between the two age groups $(\mathrm{p}>0.05)$. In the first examination, 46 patients had fistula-in-ano and 246 patients had perianal abscess. Most common symptoms were erythema at perianal region, mass, constipation, rectal bleeding and inability to defecate. Spontaneous drainage was determined in $34.9 \%(n=86)$ of the perianal abscess and $65.1 \%(n=160)$ of perianal abscess were treated with simple incision-drainage without general anestesia and systemic antibiotherapy. In the follow-up, fistula-in-ano formed in 25 patients who had perianal abscess. Spontaneously drained of abscess significantly increased the development of fistula-in-ano $(\mathrm{p}=0.001)$. Although, recurrences in perianal abscess (26\%) do not increase the fistula-in-ano formation $(p>0.05)$. In fistula-in-ano treatment, 10 patients were resolved spontaneously, 55 patients were treated with fistulotomy and 6 patients were treated with fistulectomy. Conclusion Management of perianal abscess and fistula-in-ano is still controversial. According to our results, simple incision-drainage and antibiotic usage in the treatment of the perianal abscess is the most appropriate treatment to decrease the rate of fistula-in-ano.

\section{SCREENING CRITERIA FOR COLORECTAL CANCER IN PATIENTS WITH TYPE II DIABETES MELLITUS}

\author{
Alexandra Agache, Petronel Mustatea, Traian Patrascu
}

\section{Clinical Hospital DR I Cantacuzino, Bucharest, RO}

Background/Aim Assessment and definition, using anamnestic, clinical and paraclinical criteria, the profile of the patient with type II diabetes mellitus that should be given an endoscopic examination because diagnosing precursor lesions or even colorectal cancer is likely probable. Methods Prospective case-control study conducted over a 2-year period including a number of 442 patients presented at Dr. I Cantacuzino Clinical Hospital, asymptomatic, who underwent lower digestive endoscopy; All patients included in the two groups (group 1 with type II diabetes and group 2 without diabetes) were asymptomatic in terms of digestive sphere disorders (only patients accusing abdominal meteorism and constipation were included) or in terms of general manifestations specific for neoplasia. Endoscopic findings of tumors and neoplastic precursor lesions (polyps) have been defined as positive results at colonoscopy. Thus, 52 patients with polyps $(26.80 \%)$ and 29 patients $(14.95 \%)$ with tumors in different localizations were found at the level of group 1 . Results In the analyzed group, statistically significant correlations $(p<0.05)$ were recorded between positive colonoscopy results (defined as precursor lesions-polyps- or tumors) and certain clinical characteristics (age, sex, BMI, duration of diabetes, type of antidiabetic treatment) and also paraclinical (reactive $\mathrm{C}$ protein and glycated hemoglobin).
Conclusion Criteria of patients with type II diabetes who have the maximum probability of developing colorectal cancer have been outlined. Thus this patient is more likely male, with a BMI $>25$, aged over 60 years, with an unbalanced diabetes mellitus counted by $\mathrm{HbA} 1 \mathrm{c}>7 \mathrm{mg} / \mathrm{dL}$, with over 5 years of diabetes evolution, in treatment with insulin most likely or combined insulin with oral antidiabetics and with an inflammatory biological profile expressed by $\mathrm{CRP}>2 \mathrm{mg} / \mathrm{dL}$ threshold values.

\section{SYNCHRONOUS ANAL MUCOID ADENOCARCINOMA AND ANAL TUBERCULOSIS PRESENTING AS CHRONIC ANAL FISTULA: CHALLENGING MANAGEMENT}

\author{
Sarah Benammi, Youness Bakali, Mouna Aloui, Farid Sebbah, \\ Mohamed Raiss, Abdelmalek Hrora
}

\section{University Mohamed V, Rabat, MA}

Background/Aim Anal canal cancer is a rare tumor of the digestive tract. While extra-pulmonary tuberculosis is a common case, metachronous anal tuberculosis to an anal adenocarcinoma is an exceptional condition. Each entity has well-defined therapeutic protocols. The aim of our study is to report management of the first case reported in literature of synchronized anal canal adenocarcinoma and anal canal tuberculosis. Methods 71-year-old man admitted for nonhealing anal fistula. Rectal examination at supine position showed an ulcerative growth at the medio-superior quadrant on a radius of $2 \mathrm{~cm}$ from the anal verge. Digital rectal examination assessed no tumor in the ano-rectum. Biopsy of fistulae confirmed diagnosis of anal mucoid adenocarcinoma with coexisting anal tuberculosis. Further exploration confirmed diagnosis with no distal metastasis, no active pulmonary tuberculosis and no immunodepression. Adjuvant antibacillary chemotherapy was initiated 1 month prior to adjuvant radiochemotherapy. Patient was re-admitted at the 6th week following last dose of radio-chemotherapy to undergo surgery: laparoscopic Total Mesorectal Excision. On long-term evaluation over 10 months no relapse was assessed, and the patient reported no recurrent symptoms with positive weight gain. Results Association of both entities is rare. Chronic inflammatory damage may possibly initiate a sequence of metaplasia and dysplasia, resulting in neoplastic transformation. Anal canal adenocarcima treatment follows same guidelines as rectal cancer. Extra-pulmonary tuberculosis treatment follows anti-bacillary protocol with consequent side effects. Therefore our case is a unique clinical challenge for physicians. Management decision was multidisciplinary process. We decided to undergo neo-adjuvant antibacillary chemotherapy, associated with neo-adjuvant radiochemotherapy, followed by radical resection surgery. Conclusion Their pathophysiology relationship is yet to be understood. Moreover, each entity has defined and individual therapeutic protocols and indications. All this taken into consideration, such a case creates a clinical and therapeutic challenge for physicians. The aim of our study is to report a first case in literature, and report our multidisciplinary approach.

\section{WHITEHEAD HEMORRHOIDECTOMY}

Petronel Mustatea, Alexandra Agache, Traian Patrascu

Clinical Hospita Dr I Cantacuzino, Bucharest, RO

Background/Aim Based on the experience of one surgical team, the current paper intends to reevaluate the place of this technique in the 
therapeutic arsenal of hemorrhoidal disease, starting from precise indication, accurate surgical technique and potential complications. Methods We studied 36 patients who underwent surgery between January 2013 and September 2020. Grade IV hemorrhoidal disease with irreducible mucosal prolapse was the indication for this technique. We retrospective evaluated the data on these patients such as age, gender, preoperative diagnosis and postoperative complications. Results We operated 22 male and 14 female patients with ages between 30 and 68 years (average age 51). Complications of the Whitehead procedure included urinary retention (6 cases, 16.66\%), bleeding ( 1 case with hematoma formation, $2.77 \%)$, temporary anal incontinence (1 case, $2.77 \%$ ) and mucocutaneous suture disruption (2 cases, 5.55\%). No infectious complication, ectropion or stricture developed. The average hospitalization period was 5 days. The follow-up period was between 6 and 36 months with no relapses. Conclusion In selected cases, Whitehead procedure backed by a well mastered technique provides very good long term results and low morbidity.

\section{LONG TERM OUTCOMES OF SNN IN FECAL INCONTINENCE IN A UNIVERSITY TEACHING HOSPITAL IN UK}

\author{
Sarah Khan Hull, Mona Panahi, Khalid Khan, Helen O' Grady, \\ Katherine Mockford
}

Hull University Teaching Hospital, Hull, PK

Background/Aim Fecal incontinence (FI) is a substantial health issue affecting approximately $8 \%$ of population. Sacral Nerve Neuromodulation (SNN) has been recognized as an effective treatment for fecal incontinence when conservative management has failed. SNN has shown short to medium term efficacy in treatment of FI. There have been few long term outcomes presented, often in small patient cohorts. Our aim was to present the long-term outcomes of a large cohort of patients from a single Centre followed up for at least 5 years. Methods Prospectively registered data of patients who received SNN for FI between December 2005 and December 2015, and were available for at least 5 years follow-up were analyzed. Daily stool diaries, Rockwood score, Wexner score and SF36 scores were recorded at baseline, after temporary implantation, 6 monthly, and yearly thereafter. SNS was considered successful if $750 \%$ improvement in symptoms was maintained in long term. Results Out of 335 patients who underwent temporary testing between Nov 2005 and Dec 2015,270 patients had $50 \%$ or more improvement in their symptoms and progressed to permanent implantation. 243 were available for long term follow up, with a minimum of 60 months. There was a significant reduction in episodes of FI from median 14-2, FU from median of 14-3 and Wexner score from a median of 14-7. There was also significant improvement in overall Rockwood and SF36 quality of life scores. Conclusion SNS is an effective and durable option for long term management of fecal incontinence.

\section{HIGHER RISK OF POSTOPERATIVE COMPLICATIONS IN THE UNDERWEIGHT AND OBESE PATIENTS AFTER COLORECTAL CANCER SURGERY}

Anne Fogh Juvik, Tina Fransgaard, Ole Roikjaer

Roskilde, $D K$
Background/Aim Because of conflicting evidence regarding overweight and postoperative complications this study focuses on postoperative complications and death within 30 and 90 days after curative intended surgery for CRC and the association with BMI. Methods The study included all patients who had potentially curative surgery for colon or rectum cancer in Denmark from 2014 through 2018. The primary endpoint was post-operative complications within 30 days of surgery and secondary endpoints were 30- and 90-days mortality. The post-operative complications were divided into medical and surgical complications and all clinically relevant confounders were included in a multivariate analysis. Results The cohort included 14,004 patients. In the multivariate logistic regression analysis, adjusting for relevant confounders, we found a higher relative risk of complications in all weight classes compared to normal weight. The relative risk of having a surgical complication or having both a surgical and medical complication at the same time rises with increasing weight class. The multivariate analysis showed the relative risk for both 30- and 90 days mortality is higher for underweight patients and Obesity Class III patients, but the rest of the patients have no significant differences in relative risk compared to normal weight patients. Conclusion Based on our results, there is a greater risk of surgical complications after surgery for colorectal cancer if the patient is overweight and the relative risk rises with increasing weight. The relative risk of serious complications such as anastomotic leak is also increased in the obese population.

\section{CEFUROXIME PLUS METRONIDAZOLE VS. AMOXICILLIN/CLAVULANIC ACID AS ANTIBIOTIC PROPHYLAXIS IN COLORECTAL SURGERY}

Elisavet Stavropoulou, Andrew Atkinson, Marie-Christine Eisenring, Jonas Marschall, Christoph Fux, Nicolas Troillet

Central Institute, Valais Hospital, Sion, $\mathrm{CH}$

Background/Aim The Swissnoso surveillance program (SNSP) reports surgical site infections (SSI) rates $>10 \%$ after colorectal surgery. E. coli and Enterococcus spp. are the most common microorganisms found. Despite its debated role, data suggest that the latter contributes to anastomotic leakage (and thus SSI) by producing collagenases and activating a host proteinase that breaks down collagen. Most guidelines recommend cefuroxime plus metronidazole $(\mathrm{C}+\mathrm{M})$ for the prevention of SSI in digestive surgery. Some hospitals or indivual surgeons favour amoxicillin/clavulanic acid (A/C), which, in contrast to $\mathrm{C}+\mathrm{M}$, is active against most enterocci. We aimed at determining whether $\mathrm{A} / \mathrm{C}$ prevents SSI better than $\mathrm{C}+\mathrm{M}$. Methods We used data from the SNSP from 2009 to 2018 (127 participating hospitals) to compare SSI rates between patients who received a prophylaxis with $\mathrm{A} / \mathrm{C}$ vs. $\mathrm{C}+\mathrm{M}$. Only clean-contaminated operations (class 2) were included. Co-variables analyzed were the timing of the antibiotic prophylaxis, the patients' age and sex, ASA score, operation duration, use of a laparoscope, repeat surgery, and hospital size. Multivariable logistic regression was used to adjust for potential confounders. Results Overall, SSI occurred in 2854/26,087 patients (10.9\%) receiving C + M vs. 278/1835 (15.1\%) receiving $\mathrm{A} / \mathrm{C}(\mathrm{p}<0.001)$. Intraperitoneal (organ/space) SSI occurred in $1670(6.4 \%)$ and $138(7.5 \%)$ respectively $(\mathrm{p}=0.01)$. Univariate OR of $\mathrm{A} / \mathrm{C}$ as compared to $\mathrm{C}+\mathrm{M}$ for SSI was 1.45 (CI95\%: 1.21-1.75). Other risk factors for SSI in univariate analysis were the age (OR: $1.03 ; 1.00-1.06$ per 10 years), ASA score $>2$ (1.67; $1.57-1.79)$, operation duration (1.08; $1.06-1.10$ per $30 \mathrm{~min})$, repeat surgery $(4.85 ; 3.82-6.15)$, and hospital size ( $>500$ vs. $<200$ beds) $(1.30 ; 1.07-1.58)$. Protective factors were female sex (0.67; 
$0.62-0.73)$, prophylaxis administered $30 \mathrm{~min}$. or more before incision $(0.94 ; 0.89-0.99)$, elective surgery $(0.59 ; 0.52-0.66)$, and laparoscopic surgery $(0.53 ; 0.47-0.59)$. In multivariable analysis, prophylaxis with $\mathrm{A} / \mathrm{C}$ was independently associated with higher SSI risk than with $\mathrm{C}+\mathrm{F}(1.49 ; 1.24-1.78)$. Conclusion Despite its activity against most enterococci, amoxicillin/clavulanic acid was less effective than cefuroxime + metronidazole for preventing surgical site infections after colorectal surgery. This result should be confirmed by a randomized clinical trial.

\section{HIGHER RISK OF POSTOPERATIVE COMPLICATIONS IN THE UNDERWEIGHT AND OBESE PATIENTS AFTER COLORECTAL CANCER SURGERY}

\section{Anne Fogh Juvik, Tina Fransgaard, Ole Roikjaer}

\section{Roskilde, $D K$}

Background/Aim Because of conflicting evidence regarding overweight and postoperative complications this study focuses on postoperative complications and death within 30 and 90 days after curative intended surgery for CRC and the association with BMI. Methods The study included all patients who had potentially curative surgery for colon or rectum cancer in Denmark from 2014 through 2018. The primary endpoint was post-operative complications within 30 days of surgery and secondary endpoints were 30- and 90-days mortality. The post-operative complications were divided into medical and surgical complications and all clinically relevant confounders were included in a multivariate analysis. Results The cohort included 14.004 patients. In the multivariate logistic regression analysis, adjusting for relevant confounders, we found a higher relative risk of complications in all weight classes compared to normal weight. The relative risk of having a surgical complication or having both a surgical and medical complication at the same time rises with increasing weight class. The multivariate analysis showed the relative risk for both 30- and 90 days mortality is higher for underweight patients and Obesity Class III patients, but the rest of the patients have no significant differences in relative risk compared to normal weight patients. Conclusion Based on our results, there is a greater risk of surgical complications after surgery for colorectal cancer if the patient is overweight and the relative risk rises with increasing weight. The relative risk of serious complications such as anastomotic leak is also increased in the obese population.

\section{A LONGITUDINAL STUDY OF ACUTE AND NON- ACUTE PATIENTS PRESENTING WITH HOLLOW VISCUS LYMPHOMA}

\author{
Hansen Liemangow, Muhammad Shahrukh Effendi, Malaz \\ Abdelkarim Ibrahim Abbakar, Michael Lim
}

York Teaching Hospitals NHS Trust, Scarborough, GB

Background/Aim Lymphoma of the gastrointestinal tract is rare and accounts for less than $5 \%$ of all hollow viscus malignancies. The condition may be responsible for both acute and non-acute presentations to general surgery. We chose to study the impact of this disease within a cohort of patients who presented to our services. Methods A consecutive series of patients with hollow viscus lymphoma between 2013 and 2019 were studied at York Teaching Hospitals. Retrospective case note review was performed using electronic case note review. Details on demographics, pre, peri and postoperative variables were recorded. Results There were 33 patients, 19 (57\%) presented acutely. Median age was 72 (IQR 62-80) years. Median follow up was 17 (IQR 10-45) months. Patients presenting acutely mostly had bowel obstruction and/ or perforation $(84 \%)$ requiring surgery, while those in non-acute setting mainly had paraneoplastic symptoms (57\%). Small bowel was the most common hollow viscus site, followed by colon and stomach $(55 \%, 24 \%$ and $21 \%$ respectively) Diffuse Large B-cell lymphoma was the most common subtype (66.7\%). A significant proportion of patients successfully completed their chemotherapy treatment (70\%) At maximum follow-up, $12(36 \%)$ patients had died. Median survival of those patient with acute presentations was not inferior to those patients with non-acute presentations (57 vs 49 months, $\mathrm{p}=0.38$ ). Conclusion Patients presenting to general surgery with hollow viscus lymphoma have reasonable survival on follow-up. Survival of those presenting acutely was not inferior to those presenting non-acutely.

\section{TRANSANAL TOTAL MESORECTAL EXCISION: CRITICAL APPRAISAL OF THE TECHNIQUE}

\author{
Marta Penna, Roel Hompes, Paris Tekkis, Neil Mortensen, George \\ Hanna
}

Oxford, $G B$

Background/Aim Transanal total mesorectal excision (TaTME) attracted great interest amongst the colorectal community with a rapid adoption. More recent adverse events have brought the technique into question. The aim was to evaluate TaTME technique in detail by performing Observational Clinical Human Reliability Analysis and correlate findings with post-operative outcomes. Methods One hundred unedited TaTME videos were analysed. A clinical categorisation of technical errors was devised including errors of set up/exposure, and executional errors divided into tissue-instrument interface errors and instrument-handling errors. An accuracy score from 1 to 4 was created for each subtask. The sum of the scores produces a composite objective rating with a worse performance leading to a higher score. Results were discussed at an educational seminar with 17 international TaTME expert surgeons, who provided error-reducing mechanisms and technical recommendations for TaTME. Results Overall 5101 errors and 904 adverse consequences were logged in 100 transanal operations, with a mean of $51 \pm 32.4$ errors and $9 \pm 6.6$ adverse consequences per case. A total of 191 intra-operative adverse consequences were identified by OCHRA with pursestring failure occurring 26 times. The most frequently injured structures were the anterolateral and lateral pillar neurovascular bundles $(37.0 \%$ and $35.0 \%$ ). A higher frequency of intra-operative technical errors was found to be significantly associated with an increased rate of post-operative morbidity, especially for more severe complications as Clavien-Dindo IIIa and above. A technical accuracy score above 24 was found to significantly increase the risk of post-operative complications ( $15.8 \%$ vs $42.9 \%$, Odds ratio 4.00 , 95\% CI $1.415-11.310$, p 0.007). Pursestring failure and inadequate washout of the rectum potentially risk seeding of tumour cells and subsequent local recurrence. Conclusion In conclusion, TaTME is a technically demanding operation which has the potential to allow optimal oncological resections especially in more challenging cases. However, substantial and significant errors with adverse consequences can occur leading to an increased risk of post-operative complications. Thorough structured training in the technique is essential and its true benefits over conventional approaches remain to be determined. 


\section{ROBOT-ASSISTED RADICAL PROSTATECTOMY FOLLOWING COLO-RECTAL SURGERY: A USER'S GUIDE}

\author{
Lorenzo Luciani, Daniele Mattevi, Tania Processali, Gianni \\ Malossini
}

\section{S. Chiara Hospital, Trento, IT}

Background/Aim Robotic-assisted radical prostatectomy (RARP) is the standard of care in the surgical management of localized prostate cancer: it is increasingly performed after complex abdominal and pelvic surgery. Our objective is to assess its feasibility and operative outcomes following colorectal surgery. Methods A prospective database of patients undergoing RARP is maintained at our Institution since January 2015. We reviewed all patients undergoing RARP after previous colo-rectal surgery. Demographic and clinical data, and operative outcomes, including conversion rate to open surgery and complications, were evaluated. Results Overall, 750 patients underwent RARP from January 2015 to September 2020 at our Institutions. The median age of patients at the colo-rectal and prostatectomy procedures was 60 and 68 years, respectively. (a) COLORECTAL surgery data. Thirteen RARPs $(1.7 \%)$ were performed after previous colo-rectal surgery, including resection of the rectum $(n=5)$, left colon $(n=4)$, and right colon $(n=3)$. Histopathology showed organconfined (pT0-T2) and non-organ confined disease (pT3 N1) in 7 and 3 of 12 patients, respectively (data unavailable in 2). Prostate specific antigen (PSA) was 4 or greater in $6(50 \%)$ of 12 cases at the time of colo-rectal surgery. (b) PROSTATECTOMY data. Overall prostatectomy and adhesiolysis operative time were 242 and $37 \mathrm{~min}$, respectively. Open conversion during RARP occurred in 4 of 12 patients with previous multiple or complicated abdominal surgery (aderential syndrome): in 2 cases, an ileal repair was performed. No surgical revision was performed; anemization requiring blood transfusions $(n=2)$, persistent drain output $(\mathrm{n}=1)$, and urinary tract infection $(\mathrm{n}=1)$ were reported. Histopathology showed organ-confined (pT2) and non-organ confined disease (pT3a-b) in 9 and 3 of 12 patients, respectively. Conclusion 1.6\% RARPs were performed after colo-rectal surgery. The robotic approach to prostate cancer was successful in case of previous uncomplicated colo-rectal surgery. No major postoperative complication was reported with any approach. However the risk of intestinal injury during open conversion (50\% in our series) might suggest a direct open approach in case of previous multiple or complicated abdominal surgery. A planned elective colo-rectal surgery should include a thorough urologic evaluation, as the risk of a subsequent prostate surgery was relevant in our series.

\section{ARE SCREEN DETECTED RECTAL CANCERS TRULY ASYMPTOMATIC AND HOW DO THEY DIFFER IN PRESENTATION FROM OTHER CANCERS? A SINGLE BOWEL SCREENING UNIT RETROSPECTIVE STUDY}

\section{Aya Musbahi, John Young, Anil Agarwal \\ North Tees and Hartlepool Hospital, Newcastle upon Tyne, GB}

Background/Aim Screening has reduced the mortality from colorectal cancers. Rectal cancers as a separate entity have been poorly studied in terms of screening. Rectal cancers often are associated with an array of symptoms such as bleeding, change of bowel habit, tenesmus and frequency. The primary aim of this study is to find out if screen detected rectal cancers and symptomatic rectal cancers are similar or different in characteristics. Methods We studied retrospectively at our unit, all rectal cancers from 2014 to 2019. We looked at data regarding GP presentation frequency and symptoms prior to screening appointment or to referral if symptomatic. We looked at short term outcomes and oncological outcomes. Statistical analysis was done on SPSS using a Chi squared or Fisher Exact test for categorical variables. A $\mathrm{p}$ value of $<0.05$ was considered to be statistically significant. Results 433 cancers were analysed. 379 were symptomatic and 54 were screening. There was no statistical difference in age distribution, gender distribution, mean Charlson comorbidity score, index of multiple deprivation and mean income between the groups. The symptomatic group had an average of 1.07 GP visits per patient before referral with a range of $1-4$. The screening group had an average of 0.27 GP visits per patient before referral with rectal symptoms with a range of $0-7 \quad(\mathrm{p}<0.001)$. Screening patients were more likely to report to the GP with diarrhoea, change of bowel habit and bleeding. Screening were more likely to present as T1/T2 disease than T3/T4 disease compared to symptomatic $(\mathrm{p}=0.023)$. Symptomatics were more likely to be EMVI positive $(\mathrm{p}=0.0001)$. No difference existed in tumour budding, perineural invasion or tumour deposits. Symptomatics were more likely to require levels $2 / 3$ care post op compared to screening $(\mathrm{p}=0.03)$ but there was no difference in length of stay, return to theatre, and 30 day mortality. A log rank test was run to determine if there were differences in the disease-free survival distribution for both groups. The disease-free survival distribution is statistically significantly different with $\mathrm{X}^{2}(1)=13.4, \mathrm{p}<0.05$. Conclusion More work needed to identify the patient journey and long term outcomes of screening and symptomatic rectal cancers on a national level.

\section{COLORECTAL CANCER IN YOUNG PEOPLE: DIFFERENCES ACROSS AGE GROUPS UNDER 50}

Evie Yeap, Asiri Arachchi, Ellathios Antoniou, Hanumant Chouhan

Monash Health, Dandenong, Victoria, AU

Background/Aim Recent literature has shown a rise in the incidence of colorectal cancer in patients under 50 and evidence that younger patients have a late presentation and poorer outcomes than their older counterparts. We aim to identify any differences in stratified age groups under 50 years old. Methods We utilised a prospective database of all colorectal cancer patients presenting to a single tertiary institution from July 2013 to June 2020. 135 patients under the age of 50 at time of diagnosis were then stratified into three age groups: $0-30,31-40$ and $41-50$ years old. Results There were 9 patients in the 0-30 year old group, 34 in the 31-40 year old group and 92 in the 41-50 year old group. There were more females in the 31-40 year old age group (58.8\%) compared to the youngest (44.4\%) and oldest (48.9\%) groups. $11.1 \%$ (1/9) of the youngest age group had a family history of colorectal cancer compared to $20.6 \%$ the $31-40$ year old (7/ 34 ) and $15.2 \%$ in the oldest group (14/92). Few patients had inflammatory bowel disease: 0 in the youngest group, 1 patient with ulcerative colitis and 2 with Crohn's disease in the middle group and one patient with Crohn's in the oldest group. Right sided cancers were more common in the youngest group (44.4\%) compared to $20.6 \%$ in the middle group and $13 \%$ in the oldest. Metastases at diagnosis were more common in younger age groups $(44.4 \%$ in the $0-30$ group vs. $32.4 \%$ in the $31-40$ group and $16.3 \%$ in the $41-50$ group). The younger groups more commonly underwent an emergency operation: $44.4 \%$ in the youngest group, $17.6 \%$ in the $31-40$ year olds and $9.8 \%$ in the oldest group. Stage IV cancers at time of resection were more common in the youngest group $(33.3 \%)$ compared to the oldest $(3.3 \%)$ and middle groups (17.6\%). Lymph node involvement occurred in $66.7 \%(6 / 9)$ of the youngest group, compared to $38.2 \%$ $(13 / 34)$ of the middle group and $37 \%$ (34/92) of the eldest group. Lymphovascular invasion was also more frequent in the youngest 
group at $33.3 \%$, compared to $23.5 \%$ in the middle and $20.7 \%$ in the oldest. In the oldest group, $66.3 \%$ of patients were alive without recurrence at the end of the study compared to $52.9 \%$ in the 31-40 years group and $33.3 \%$ in the youngest. Conclusion Younger patients with colorectal cancer present at a later stage, have poorer prognostic factors at resection and have corresponding worse outcomes.

\section{PERI-OPERATIVE OUTCOMES OF ERAS IN INFLAMMATORY BBOWEL DISEASE: A RETROSPECTIVE COHORT STUDY}

\section{Rebecca Lendzion, Ankur Sidhu, Basil D’Souza \\ St Vincent's Hospital Melbourne, NSW, AU}

Background/Aim Inflammatory bowel disease (IBD), including Crohn's disease (CD) and Ulcerative Colitis (UC), is characterized by chronic relapsing, and remitting gastrointestinal inflammation. Despite the range of immunosuppressive medication, rates of surgery remain high. Enhanced recovery programs are evidence-based protocols designed to standardise medical care, improve outcomes, and lower health care costs. Data from observational studies and randomized trials show that ERAS protocols in colorectal (CR) surgery are associated with reduced length of stay (LOS) and morbidity, faster recovery and comparable or reduced readmission rates compared to traditional care. These studies are typically performed in patients undergoing surgery for CR cancer. Currently, there is little evidence assessing outcomes of ERAS protocols in CR resection for IBD. We hypothesised that use of an ERAS protocol in IBD patients is associated with short LOS and low morbidity. Methods A retrospective review of 119 patients undergoing abdominal surgery at a high volume IBD tertiary referral centre with a well-established ERAS protocol. Data from eligible patients were recorded in a data base designed for teaching and research purposes. Results During the study period (2013-2019), 119 patients with IBD (74 CD, 45 UC) underwent surgery. Mean age was 38 years (range 18-79 years). Mean BMI in CD patients was $26.1 \mathrm{~kg} / \mathrm{m}^{2}$ (range: $14.9-44 \mathrm{~kg} / \mathrm{m}^{2}$ ) and $25.2 \mathrm{~kg} / \mathrm{m}^{2}$ (range: $18-4125.2 \mathrm{~kg} / \mathrm{m}^{2}$ ) in UC patients. Eighty patients (67\%) were allocated to an ERAS protocol; compliance was 70\%. The ERAS cohort were more likely to have laparoscopic surgery (63\%), compared to the non-ERAS cohort (N-ERAS), $(55.5 \%)$. Mean operating time was shorter in the ERAS cohort (230 min) compared to the N-ERAS group (257.5 min). Mean time to flatus and bowel motion was faster in the ERAS cohort. Mean hospital stay was significantly shorter in the ERAS cohort compared to the N-ERAS cohort (7.5 vs 11.5 days; $p<0.001$ ). Complication rates were higher in the N-ERAS cohort (62\% vs. $20 \%$ ). The 30-day readmission rate was twice that seen in the N-ERAS cohort. Multivariate regression to evaluate non compliance with ERAS is planned. Conclusion This study demonstrates that use of an ERAS protocol in IBD patients undergoing small or large bowel resection, results in favourable short-term outcomes-reduced LOS, faster time to return of bowel function and low morbidity.

\section{EMERGENCY ROBOTIC COLORECTAL RESECTIONS-A CASE SERIES}

Qiang Lu, Samuel Stefan, Emma Rawlinson, Patricia Stevens, Gerald David, Jim Khan

Portsmouth, GB

Background/Aim Robotic colorectal surgery is rapidly evolving as it addresses many of the technical and the ergonomic limitations of laparoscopic surgery. The precision of robotic surgery results in smaller incisions, shortened hospital stay, less postoperative pain, and a much quicker return to normal, thus significantly improving patient experience. However, the application of robotic surgery in the emergency setting remains very limited due to the logistical and organisational challenges, and reluctance in adoption by the clinical teams.The aim of this case series study was to report the outcomes and early experience of emergency robotic colorectal surgery at our institution. Methods All consecutive patients having emergency robotic colorectal surgery at our institution over a 12-month period (October 2019 to September 2020) were recruited in this study. Data were collected from the electronic patient records. Results Five patients ( 3 female) were included in the case series with a median age of 68.8 years (36-83). Median BMI was 27 (range 19-41). All patients were admitted to the emergency surgical unit with an acute presentation. Three patients underwent robotic right hemicolectomy, with complete mesocolic excision for obstructing right sided colon cancers. One patient had robotic anterior resection of a colo-vesical fistula secondary to diverticular disease with a pelvic abscess, and the other had robotic subtotal colectomy for acute toxic colitis following failure of medical therapy. All cancer patients had R0 resection. Median lymph node count was 48 , and the median operating time was $212 \mathrm{~min}$ (range 120-350 min) There were no grade III/IV complications postoperatively and no 90-day mortality. One patient developed surgical site infection, treated with antibiotics. Conclusion This case series highlighted that robotic colorectal surgery could achieve favourable outcome for patients with an emergency admission, with acceptable operating times. Well led clinical teams with appropriate training can offer the benefits of robotic surgery to this challenging group of patients.

\section{A CASE OF STENOTIC SIGMOID COLON CANCER WITH MASSIVE LIVER METASTASIS AND MULTIPLE LUNG METASTASES SUCCESSFULLY TREATED WITH MULTIMODAL TREATMENT INCLUDING CHEMOTHERAPY AND LIVER FIRST RESECTION}

\section{Hitoshi Idani}

Hiroshima City Hiroshima Citizens Hospital, Hiroshima, JP

Background/Aim A successful case of stenotic sigmoid colon cancer with massive liver metastasis and multiple lung metastases successfully treated with multimodal treatment including chemotherapy and liver first resection is presented. Methods A 61-year-old man presented to our hospital with a chief complaint of abdominal distension. Computed tomography showed multiple tumors in the bilateral lung and massive tumor of the right and middle lobe of the liver. Dilated small intestine and colon and a tumor at the sigmoid colon were also confirmed. Sigmoid colonoscopy showed stenotic sigmoid colon cancer. He was diagnosed as stenotic sigmoid colon cancer with massive liver metastasis and multiple lung metastases and transverse colostomy was created by emergent surgery. Histology by biopsy during sigmoid colonoscopy showed tubular adenocarcinoma and RAS was wild type. Sigmoid colon cancer and lung metastases were resectable, however, liver metastases were judged not to be resectable by volumetry. Results Accordingly, chemotherapy of FOLFOX + panitumumab was induced. After nine times of chemotherapy, lung and liver metastases markedly reduced in size and CEA and CA19-9 were surprisingly reduced from 5029.0 to 37.9 and $10,851.0$ to 7.9 , respectively. The extended right lobe resection with choledochojejunostomy was performed first and liver metastasis 
was completely resected. Then the sigmoid colon resection and taking down of the transverse colostomy were performed followed by the resection of the lung metastases.After surgery, CEA and CA19-9 were reduced within the normal range. Additional resection for liver S3 metastasis and lung metastases arising after the initial resection were performed. During follow up period of three years after the last resection of the lung metastases, there have been no recurrences at all. Conclusion Our strategy including chemotherapy and liver first resection for stenotic sigmoid colon cancer with massive liver metastasis and multiple lung metastases was effective.

\section{SARCOPENIA AND ITS EFFECTS ON SURGICAL OUTCOMES IN ELDERLY PATIENTS WITH COLORECTAL CANCER}

Jingting Wu, Frederick Koh, Caroline Loh, Winson Tan, Leonard Ho, Dulcena Yen, Sharmini Su, Fung Joon Foo, Min Hoe Chew

Sengkang General Hospital, Singapore, SG

Background/Aim Sarcopenia, characterized by progressive and generalized loss of skeletal muscle mass and strength is associated with poorer outcomes in cancer patients. The aim of this study is to examine the effects of sarcopenia on surgical outcomes in elderly patients with colorectal cancer. Methods Elderly patients $>1$ $=70$ years old undergoing colorectal cancer resections were prospectively recruited from a single institution. Skeletal mass index was measured retrospectively on CT scans with the measurement of the average Hounsfield Unit (HU) of bilateral L3 psoas muscles. Patients were separated into those at risk of sarcopenic and those who are not. Differences in terms of demographics, post-operative complications were examined. Results Eighty patients from a single tertiary institution, with a median age of 77.5 (70-90) years old were recruited between 2016 and 2020. Overall incidence of individuals at risk of sarcopenia was $70 \%$. Baseline characteristics between the groups were similar. There was no significant difference of sarcopenia on morbidity (OR 1.19; 95\% CI 0.272-5.212; p = 0.817), after multivariate analysis correcting for gender and stage of disease (OR $1.047 ; 95 \%$ CI $0.221-4.953, \mathrm{p}=0.953)$. Conversely, stage of disease confers a higher morbidity risk (OR 2.336; 95\% CI $0.55-9.918$, $\mathrm{p}=0.25)$ despite not reaching statistical significance. Conclusion In the elderly population, sarcopenia was not a big factor in determining morbidity as compared to stage of disease.

\section{TUMOR SIZE IS A BETTER PREDICTOR OF SURVIVAL \\ THAN THE CLINICOPATHOLOGICAL STAGE OF COLORECTAL CANCER}

\author{
Ali Mourad, Marie Shella De Robles, Sharlyn Kang
}

\section{The Wollongong Hospital, New South Wales, AU}

Background/Aim The American Joint Committee on Cancer has developed a framework for the clinicopathological staging of colorectal cancer based on the TNM classification system. Traditionally, earlier stages of the disease were thought to carry a more favorable prognosis; however several multicenter studies have reported superior 5-year overall survival for stage IIIA cancers when compared to stage IIB/C - the so called "survival paradox". The aim of this study was to assess whether the survival paradox was present across an Australian health district and to investigate the factors that may explain this paradox. Methods Data was retrospectively analyzed on 161 rectal cancer patients treated and staged across our health district. Our primary end-point was cancer specific survival. Results 161 rectal cancer patients were included, of which 91 were stage IIA, 20 were stage IIB/C and 50 were stage IIIA. The median follow up was 45 months. The 5-year cancer specific survival was $79 \%, 51 \%$ and $86 \%$ for stage IIA, stage IIB/C and stage IIIA, respectively. On Kaplan-Meier analysis, there was a significant survival difference between IIB/C and IIIA cancers $(\mathrm{p}<0.05)$. A subgroup analysis was performed for stage IIB/C rectal cancers depending on the number of lymph nodes retrieved intraoperatively and whether they received adjuvant chemotherapy. Even when this group received optimal treatment (i.e. $>12$ nodes retried and adjuvant chemotherapy given), 5-year survival for stage IIB/C cancers was still less than that of stage IIIA rectal cancers $(71 \%$ vs $82 \%)$. On univariate analysis, pre-operative CEA and receipt of adjuvant chemotherapy correlated with cancer specific survival (p-value 0.001 and 0.009 , respectively). Patient's with IIB/C cancers were also more likely to be symptomatic from their disease at the time of presentation. Conclusion Our study affirms the presence of the survival paradox of colorectal cancers in an Australian population. Consideration should be given to placing greater emphasis on tumor size as a prognostic factor when staging colorectal cancer.

\section{NEW APPROACH WAVELENGTH FOR HEMORRHOIDS TREATMENT OF A 1940 NM DIODE LASER TISSUE COAGULATION- EXPERIMENTAL STUDY}

\author{
Donatas Danys, Eugenijus Jasiunas, Rolandas Palubeckas, Julius \\ Pacevicius, Zbigniew Rybak, Tomas Poskus
}

Vilnius University Hospital Santaros Klinikos, Vilnius, LT

Background/Aim The aim of this study is to delineate the size of coagulation effect of diode $1940 \mathrm{~nm}$ laser on perianal tissue model depending on laser power and laser activation time and no any reports in literature about $1940 \mathrm{~nm}$ laser are using on human. The benefits of the experimental study are significant, since the data obtained during the study will allow objectification of the laser effect on soft perianal and submucosal tissues. It will help to determine the best parameters of laser. Methods Experimental study of Diode laser TWIST $1940 \mathrm{~nm}$ impact on soft perianal tissues. Rectal tissue of 30 the freshly slaughtered pigs were harvested $5 \mathrm{~cm}$ in depth around anus. 53 samples were produced and randomly assigned to receive laser power of 4, 5, 6 and $8 \mathrm{~W}$ and 3 or $6 \mathrm{~s}$ pulses. Procedure was made using Metrum diode laser TWIST. Each sample will be exposed by TWIST $1940 \mathrm{~nm}$ diode laser with different settings. All manipulations were performed by the same team lead by one surgeon, experienced in laser hemorrhoidoplasty procedure. The insertion place was marked with a pin for identification during the pathological examination. The length of tissue injury was measured on high magnification microscopy. Results The deepest tissue injury (mean $7.6 \mathrm{~mm})$ was caused by the longest laser exposure time $(6 \mathrm{~s})$ and power $(8 \mathrm{~W})$. The depth of tissue damage within various laser power groups varies on average from $4.3 \mathrm{~mm}$ to $7.6 \mathrm{~mm}(\mathrm{p}=0.01)$. The optimal difference in length of tissue damage between different power and intensity we found in groups of $18 \mathrm{~J}$ damage, it was statistically significant $(\mathrm{p}<0.05)$. Conclusion The effect of laser depends on laser exposure time and power and at $6 \mathrm{~W} 3 \mathrm{~s}$ continuous wave pulses seem to produce the coagulation length of $4.4 \mathrm{~mm}(\mathrm{p}<0.05)$. They showed most effective damage of tissue comparing with other parameters. 


\section{NO EVIDENCE FOR DIFFERENCES ON THE KINETICS OF CANCER RECURRENCE AFTER LAPAROSCOPIC VS. OPEN SURGERY IN COLON CANCER. A META-ANALYSIS} Yeh, Nicklaus Ng, Ross Tulloch, Amber Orr, Marcin Szostok, Patrice Forget

Tirana, $A L$

Background/Aim Colorectal cancer (CRC) is a leading cause of mortality in the UK and worldwide. Surgical resection is the only curative treatment modality available and using a laparoscopic vs. an open approach may have a direct influence on the inflammatory response, influencing cancer biology and potentially the recurrence kinetics. Many existing systematic reviews have compared outcomes after laparoscopic versus open surgery for CRC, but few focus solely on colon cancer and did not specifically look at the recurrence kinetics. This systematic review aims to compare laparoscopic surgery with open surgery for the treatment of colon cancer with a specific focus on the moment of the recurrence. The outcomes compared are the time to recurrence and the overall survival (OS) and disease-free survival (DFS) at 3 and 5 years. Methods A systematic review of Ovid MedLine, Ovid Embase, Cochrane Central Register of Controlled Trials and PubMed was conducted, with defined inclusion and exclusion criteria used for screening. Data was extracted from these papers and the studies were critically appraised and assessed for risk of bias. The GRADE assessment was used to determine quality of evidence. We performed meta-analysis for comparison of means for continuous data using RevMan V. 5.4.1 software. Given the hetereogeneity of studies we used the random effect method. Results Ten randomised controlled trials were reviewed. Most of the trials did not display a statistically significant difference in either DFS or OS at three or at five years when comparing laparoscopic to open surgery. A meta-analysis revealed no difference between groups in the OS and DFS, especially regarding the time needed to observe the median recurrence rate. Conclusion This review shows that there appears to be no difference in the recurrence kinetics, OS or DFS at three or five years when comparing laparoscopic to open surgery in colon cancer. This does not support an effect of the surgical approach on the cancer biology.

\section{DEMOGRAPHIC AND CLINICAL PROFILE OF VERY YOUNG COLORECTAL CANCER PATIENTS-AN INDIAN EXPERIENCE}

\author{
Kunal Dhall, Prakash Bhagat, S.V.S Deo, Sandeep Bhoriwal, Sunil \\ Kumar, Atul Sharma, Sushmita Pathy
}

\section{All India Institute of Medical Sciences, New Delhi, IN}

Background/Aim Colorectal cancer (CRC) is the third most common cancer but the median age at diagnosis of $\mathrm{CRC}$ is quite variable. The last two decades experienced a rise in incidence of CRC in very young patients (VYCRC), i.e. less than 30 years of age. There is a scarcity of literature related to VYCRC from low and middle-income countries (LMIC). In this study, the incidence, demographic, and clinical profile of VYCRC patients were analyzed. Methods A retrospective analysis of prospectively maintained colorectal cancer database was performed and data of histopathologically proven very young colon and rectal cancer patients (below 30 years of age) operated between January 1994 and December 2019 was extracted. An analysis was performed for the proportion of VYCRC, demographic and clinical profile, treatment patterns, and oncological outcomes. Results A total of 970 CRC patients were operated on during the study period and out of these, 401 (41.3\%) had colon cancer and $569(58.7 \%)$ had rectal cancer. A total number of 159 (16.4\%) patients were qualified for inclusion as VYCRC. The proportion of very young rectal cancers (VYRC), i.e.? 30 years of age was higher $(22.3 \%)$ in contrast to very young colon cancers (VYCC) $(8 \%)$. The male-to-female ratio was 1.9 and 1.8 in VYCC and VYRC, respectively. Only 6 patients each with VYCC (18.8\%) and VYRC (4.7\%) had a positive family history. The VYCC had equal involvement of the ascending colon and sigmoid colon $(40.6 \%)$ whereas the lower rectum was most commonly involved subsite in VYRC patients (63\%). The majority of VYCC patients presented with stage 2 disease $(53 \%)$ and VYRC patients with stage 3 disease $(58 \%)$. Among VYRC patients neoadjuvant treatment in the form of shortcourse radiation or long-course chemoradiation was used in 35 $(27.6 \%)$ and 47 (37\%) patients respectively. The curative resection rate for VYCC was $65.6 \%$ and VYRC was $51.2 \%$. After curative resection, 4/21 (19\%) and 46/65 (70.8\%) patients with YCC and YRC developed relapses. Conclusion The results of the current study indicated that VYCRC constitutes a significant proportion of the CRC burden. Among VYCRC, rectal cancer was more common than colon cancer. The majority presented with a locally advanced stage and had modest curative resection and disease control rates. VYCRC seems to be a distinct entity warranting future basic and clinical research.

\section{IMPACT OF COVID-19 PANDEMIC ON COLORECTAL CANCER SURGERY IN INDIA}

\section{Hemant Kumar Singh,Vijayraj Patil, Ganne Chaitanya}

All India Institute of Medical Sciences, Mangalagiri, IN

Background/Aim COVID-19 has impacted cancer care across India. This study aims to understand changes in colorectal oncologic practices particularly with regards to the selection of preoperative radiotherapy (RT) in colorectal surgery, the functioning of RT centers, and PPE for high-risk procedures across India at the time of nationwide lockdown. Methods A questionnaire-based online survey was conducted in May 2020 (phase 4 of nationwide lock-down) among surgical oncologists practicing in India. Results A total of 208 oncologists in 188 centers in 70 cities completed the survey. $66.5 \%$ of oncologists found it difficult to stage the patients for surgery at the time of the lockdown. $18.7 \%$ of oncologists preferred short-course RT (SCRT) over long course RT (LCRT), LCRT over SCRT by $43.3 \%$. Almost $40 \%$ of the oncologists did not have any preference and continued the practice as before. There was no significant difference for preoperative RT between the tier of the city, type of organization (government vs private), and green/red zones at the time of the lockdown. Open surgeries were preferred over minimally invasive surgeries (MIS) $(64.2$ vs $11 \%)$ especially in tier 3 cites $(p=0.07)$ and academic centres $(\mathrm{p}=0.008)$. Cytoreductive surgery/hyperthermic intraperitoneal chemotherapy (CRS/HIPEC) was practiced by fewer surgeons $(30.5 \%)$. Oncologist reported RT center shut down $(4.2 \%)$, increased waitlist $(24.5 \%)$, and use of hypofractionated RT $(38.3 \%)$ and palliative RT continuation $(81.9 \%)$. The availability of PPE to operate on suspected COVID patients $(93.3 \%)$, for evaluating and operating in an emergency (89.5\%), and (3) for endoscopy (88\%) did not differ significantly between the tier of the city, type of organization and zones across the country. Conclusion Pandemic has impacted the colorectal surgeons' practices and RT centers functioning centers across the country. Onco-surgeons preferred LCRT over SCRT and an equal number did not change their prior preferences even at the time of the pandemic. Colorectal surgeons adopted 
by using PPE, and changing approaches for the surgery to balance patient care and the risks associated.

\section{MESH PROPHYLAXIS FOR INCISIONAL HERNIA: A SYSTEMATIC REVIEW AND META-ANALYSIS}

\section{Dhruvesh Ramson, Hugh Gao}

\section{Monash University, Auckland, NZ}

Background/Aim To determine by meta-analysis if prophylactic mesh augmentation compared to primary suture closure alone can reduce the risk of incisional hernia in patients undergoing abdominal surgery. Methods We searched multiple databases for randomised clinical trials comparing prophylactic mesh augmentation against primary suture alone during abdominal surgery in preventing incisional hernia. Per-protocol and intention-to-treat risk ratios were calculated for primary outcomes. Per-protocol risk ratios were calculated for secondary outcomes. Meta-analysis was performed using the MH-method as implemented by the metafor package. Results Fifteen distinct randomised controlled trials involving 2326 patients were identified and were subsequently included in this meta-analysis. Data from fifteen RCTs all individually trend towards a reduction in risk of incisional hernia with placement of prophylactic mesh. The pooled risk ratio for incisional hernia for all studies was 0.46 (95\% CI 0.39-0.55) Leave-one-out sensitivity analysis was performed to show that statistical significance was maintained independent of any one particular study being excluded from meta-analysis. The insertion of mesh compared against conventional suture bulk closure did not increase the risk of wound infection, as demonstrated by the RR of 1.22 (95\% CI 0.88-1.69). Conclusion Placement of prophylactic mesh compared to primary suture repair alone at time of abdominal surgery reduces the risk of developing incisional hernia. This metaanalysis should increase the propensity of surgeons to offer mesh prophylaxis to patients undergoing abdominal surgery, particularly in the presence of risk factors of developing incisional hernia.

Management of low rectal cancer in Monastir-Tunisia: study of 24 cases in 10 years? Khadija Zouari, Besma Mnajja Department of general and digestive surgery Monastir-Tunisia Abstract Introduction: the prognosis of low Rectal Cancer (LRC) has imp

\section{Limayem Zouari Khadija}

Sousse Tunisia, TN

Background/Aim The prognosis of low Rectal Cancer (LRC) has improved in recent years. The combination of the neoadjuvant chemoradiation treatment (CRT) and total mesorectal exicision (TME) has increased the overall survival and it have reduced the rate of local recurrence. However, functional sequelae after curative treatment remain a substantial problem. Methods It is a retrospective study that was carried on the department of visceral and digestive surgery in the Fattouma Bourguiba Hospital Monastir-Tunisia, concerning Low Rectal Cancer (LRC), between January 2004 and December 2013. Results Twenty four patients with LRC were reviewed. The mean age of patients was 55 years. Male ascendancy was noted in $66 \%$ of cases. The mean diagnostic delay was 7 months. The most found clinical symptoms were: a Rectal Bleeding (18 cases), a Rectal syndrom (15 cases), an Intestinal motility disorder (17 cases) and a changing of the general state (13 cases).The digital Rectal examination (DRE) was made in all cases and it showed a tumoral lesion. The rectoscopy was practiced in $100 \%$ of cases, allowing to take biopsy which confirmed the diagnosis of Low Rectal Cancer (LRC): adenocarcinoma (20 cases) and epidermoid carcinoma
(4 cases). The pretherapeutic assessement included CT scan (chest scan, abdominal scan, pelvic scan) which was practiced in $85 \%$ of cases and MRI (23\% of cases). The Endoscopic Rectal Ultrasongraphy was not made in any case. Nine percent of the sick had metastasis. All the patients had Radiochemotherapy before the surgery. In the majority of cases, the surgical way was median. However, laparoscopic surgery was practiced in $14 \%$ of cases. The surgical procedure was an Abdomino-perineal Resection (APR) in $72 \%$ of the cases. The rest of patients had a stoma of discharge (dump). The adjuvant treatment was composed of Radiotherapy (9\% of cases) and Chemotherapy (19\% of cases).The mortality rate was $19 \%$. The local recurrence was detected in $9 \%$ of cases. Metastatic evolution was noted in 3 cases. Conclusion Low Rectal Cancer is a serious problem of Health in the world. The two goals of surgery of this disease is to obtain clear "curative " margins and to limit post surgical functional disorders. Actually, Neoadjuvant Radiochemotherapy only can have good results in realizing a complete pathological Response (pCR).

\section{ASSESSMENT OF RECTAL POUCH CLOSURE IN SUBTOTAL COLECTOMIES FOR SEVERE ACUTE COLITIS: SINGLE STOMA APPROACH}

\section{Youness Bakali, Sarah Benammi, Mouna Aloui, Farid Sebbah, Mohamed Raiss, Abdelmalek Hrora}

\section{University Mohamed V, Rabat, MA}

Background/Aim Standard surgical care for Severe Acute Colitis is subtotal colectomy with ileostomy. However, the management of the rectal pouch remains controversial. Closure of the rectal pouch offers the advantage of having a single stoma and preventing infection of abdominal wall. Therefore the aim of our study is to assess the morbidity and mortality of this technique. Methods A single-center retrospective study conducted between 2014 and 2019, including patients admitted for surgical care of Inflamatory Bowel Disease undergoing subtotal colectomy for Severe Acute Colitis. Three groups of patients were compared: Subtotal colectomy with double stoma (G1), subtotal colectomy with sigmoid pouch placed subcutaneously (G2), and subtotal colectomy with rectal pouch placed in the pelvis (G3). The diagnosis of Sever Acute colitis was retained on a TrueLove and Witts score greater than 9. And indication for emergency surgery was retained upon failure of medical treatment for 5 consecutive days or in the presence of a serious factor; resistance to medical treatment, Haemorrhage, toxic megacolon. Results 35 patients were included. Median age was 34 years [28.5-47]. 60\% were men. Mean BMI was $19.25 \pm 1.8$. The median duration of the disease was 24 months [6-54]; $91.7 \%$ of the patients were on oral corticosteroids, $50 \%$ were on salazoperines, and $30.4 \%$ were on immunosuppressants (Methotrexate). Resistance to medical treatment was assessed in 50\%, 30.8\% reported haemorrhage, and $19.2 \%$ were megacolon cases. $71 \%$ of cases were laparoscopic surgery. Subtotal colectomy with double stoma (G1) was completed in $42.9 \%$ of cases, $28.6 \%$ with sigmoid pouch $(\mathrm{G} 2)$, and $28.6 \%$ with rectal pouch $(\mathrm{G} 3)$. The median operating time was 195 min [123-315]. The postoperative evolution was favorable in $78.4 \%$; all groups included. Study assessed $12 \%$ of intestinal occlusion, and intra-abdominal collections in $6 \%$. Mortality rate was $10.7 \%$. The study found no statistical significant difference at comparison of the postoperative complication between the three groups. Conclusion Despite the retrospective nature and the small number of patients in this study, we observed that the occurrence of an infectious complication, in particular pelvic sepsis on a rectal pouch is low, offering the possibility of having a single stoma and avoiding the risk of abdominal wall infection. 


\section{COLONIC STENTING AS A BRIDGE TO SURGERY IN OBSTRUCTING COLORECTAL CANCER. FIVE YEARS' EXPERIENCE FROM TERTIARY REFERRAL CENTRE}

\author{
Mahwish Khawar, Mahmood Dhaheri, Ibrahim Amer, Muniba Afzal, \\ Mohammed Yousif, Mohamed Abunada, Amjad Parvaiz
}

Hamad General Hospital, Hamad Medical Corportation, Doha, QA

Background/Aim Colonic stenting is used as an alternate way of ameliorating obstructive symptoms in the management of acute colonic obstruction. When successfully performed; it provides potential advantage to convert emergency to elective procedure leading to better short-term outcomes. We present our experience of utilization of colonic stent as bridge to surgery from single centre practice over five years. Methods Data was collected for all patients presenting between January 2015 and September 2020 with diagnosis of colorectal cancer in largest tertiary referral colorectal department of the country. All patients with obstructing colonic malignant lesions located between transverse colon and rectosigmoid region were included. Analysis performed for patients who underwent surgical resection with curative intent following the attempted stent procedures. It includes mode of surgery, stent placement success rates, length of stay, stoma rates, conversion and morbidity and mortality associated with surgery. Results Total of 485 patients with colonic cancer were treated at our institution. 44/485 (9\%) required emergency endoscopic stenting to relieve colonic obstruction. 35/44 (79\%) patients had successful clinical response to stenting. 14/44 (32\%) had procedure performed with palliative intent and did not require surgery. 21/44 (48\%) underwent elective procedure while the remaining $9(20 \%)$ patients required emergency surgery. The median time for the elective resection following stent placement was 6 weeks (range 4-8 weeks). Laparoscopic resection was performed in 19/21 (90\%) of elective resections with $9 \%$ conversion rate to open surgery. The results for both elective and emergency resections are shown below. Elective surgery $\mathrm{n}=21$ Emergency Surgery $\mathrm{n}=9$ Male $14(67 \%) 6$ (67\%) ns Age (median) 54 (41-75) 53 (22-78) ns Stoma 4 (19\%) 7 (78\%) (P less than 0.0025) Leak $3(14 \%) 0(0 \%)$ ns Disease free 11 $(52 \%) 1(11 \%)$ ( $\mathrm{P}$ less than 0.0374$)$. Conclusion Colonic stenting is associated with higher success rates for the relief of obstruction. It provides an opportunity to convert emergency to elective resection leading to significantly lower stoma formation, shorter length of stay and better disease-free survival in our study.

\section{ROBOTIC APPROACH IN COMPLICATED DIVERTICULAR DISEASE MAY REDUCE BLOOD LOSS AND AVOID A DIVERTING STOMA}

Ioannis Mykoniatis, Francesca Pecchin, Samuel Stefan, Najaf Siddiqi, Vicky Maertens, M. Wagh, S. Naqvi, K. Flashman, Jim Khan

\section{Queen Alexandra Hospital, Porthsmouth, GB}

Background/Aim Surgery remains the treatment of choice for complicated colonic diverticular disease (DD). The laparoscopic approach is increasingly being used due to its well-established shortterm clinical benefits. We aimed to investigate the safety, effectiveness and advantages of a robotic approach compared with a fully laparoscopic approach in the surgical management of complicated DD. Methods Between January 2014 and March 2019 all consecutive patients presenting with complicated DD (defined using set criteria) and having a minimally invasive elective sigmoid resection were included. Data were collected from a prospectively-maintained, ethically-approved hospital database. Patient demographics, operative findings and postoperative outcomes were recorded. We retrospectively reviewed patients who had a restorative anterior resection. Patients with a stoma only or a Hartman's resection were excluded, as were all emergency resections. Results During the study period 107 patients had minimally invasive sigmoid resection for complicated Diverticular disease. Thirteen patients were excluded (stoma only or a Hartman's procedure). Overall, 94 patients underwent colorectal resections; of these, the laparoscopic approach was used in 75 patients, and the robotic approach in 19 patients. Use of the robotic approach compared with the laparoscopic approach resulted in a comparable mean operative time ( 238.9 vs. $260 \mathrm{~min}$, respectively) but significantly lower mean intraoperative blood loss (20 vs. $140 \mathrm{ml}$; $p=0.005$ ). Conversion to open surgery was required in five laparoscopic cases, but no robotic cases. Significantly more temporary diverting stomas were performed using laparoscopy [61 (81.3\%) patients] than the robotic approach [four $(21 \%)$ patients] $(\mathrm{p}<0.001)$. The median hospital stay was also comparable between groups ( 7 for robotic vs. 8 days for the laparoscopic group) Anastomotic leak occurred in $6.6 \%$ of laparoscopy patients, but no robotic patients. In two laparoscopic cases, re-intervention was required. Conclusion Our preliminary experience highlights the potential benefits of robotic surgery in the management of complicated diverticular disease in terms of safety, efficacy and postoperative outcomes. The robotic technique can also decrease the rate of protective ileostomy creation, hence reducing common complications related to diverting stoma.

\section{POSTOPERATIVE KIDNEY INJURY AFTER RECTAL RESECTION AND ALL-CAUSE MORTALITY}

Joseph Borucki, Alexandra Fielding, Rebecca Woods, Lucy-Anne Webb, James Hernon, Simon Lines, Adam Stearns

\section{Norfolk and Norwich University Hospitals, NHS, Norwich, GB}

Background/Aim Kidney injury is a common complication of colorectal surgery affecting 6-20\% of patients. Whilst the effect on short term mortality is well defined, there has been no assessment of the long-term effect of kidney injury on mortality and renal function in patients undergoing rectal resection for malignant disease. This study aims to assess these effects. Methods This is a retrospective single site cohort study, examining all patients who underwent rectal cancer surgery between January 2003 and March 2017. Preoperative eGFR was compared to postoperative eGFR and patients were divided into cohorts based on decline in renal function from baseline, with $25 \%$ and $50 \%$ decline threshold corresponding to RIFLE R and I grade. Cox-proportional hazards regression analysis was performed between the cohorts. Kaplan-Meier analysis was performed between the cohorts and the curves compared with log-rank analysis. Trends in renal function were performed with linear regression analysis. Results 1159 patients were identified as eligible for inclusion into the study. Adjusted hazard ratios for post-operative deterioration in eGFR of $>25 \%$ and $>50 \%$ were $1.72(1.15-2.55, \mathrm{p}=0.007)$ and 2.74 $(1.40-5.36, p=0.003)$ respectively. A $>25 \%$ reduction in eGFR was associated with reduced median survival from $144.5(128.1-160.9)$ to 86.0 (64.0-108.0) months, whereas a $>50 \%$ decline was associated with a reduction to 53.3 (7.8-98.8). Early postoperative renal impairment was irreversible: patients with a $>25 \%$ postoperative reduction in eGFR had a mean decline at 10 years of $38.8 \%$, compared to $10.2 \%$ for those who did not. Conclusion Even modest reductions in renal function following colorectal surgery have been demonstrated to significantly increase long term mortality. There is also a significant effect on long term renal function. Research into 
protecting against kidney injury postoperatively should be explored as this represents a potential way to significantly improve long term survival.

\section{WATCH AND WAIT FOR RECTAL CANCER-ARE PATIENTS WITH LOCAL REGROWTH AT RISK FOR DEVELOPMENT OF DISTANT METASTASES?-A CONDITIONAL SURVIVAL ANALYSIS}

Laura Fernandez, Guilherme São Julião, Angelita Habr-Gama, Nuno Figueiredo, Bruna Vailati, Geerard Beets, Andrew Renehan, Cornelis Van de Velde, Rodrigo Perez

\section{IWWD International Watch \& Wait Database Consortium}

\section{Fundação Champalimaud, Lisbon, PT}

Background/Aim Watch and Wait (WW) for rectal cancer patients with clinical complete response (cCR) after neoadjuvant treatment (nCRT) remains controversial. Nearly $30 \%$ will develop a local regrowth (LR) during the first 3 years of follow-up. Although the vast majority of LRs have been managed with successful salvage surgery and acceptable local disease control, these patients may be at higher risk for development of distant metastases (DM). We aimed to investigate the association of LR and DM and the effect of LR in the risk of DM over time. Methods Data from the International Watch and Wait Database (IWWD) of all patients with cCR after nCRT managed non-operatively was retrospectively reviewed. Cox logistic regression was used to determine independent risk factors for worse distant metastases-free survival (DMFS). Conditional survival modelling was used to investigate the impact of local regrowth in the development of distant metastases over time. Time to event was calculated using two different scenarios for time zero to the diagnosis of DM: the date of decision for WW (1) and the date of LR (2). Results 793 patients who achieved cCR after nCRT were included. LR was the only statistically significant risk factor for worse DMFS (HR 4.34 95\%CI 2.16-8.71). Using conditional estimates, LR remains as a significant risk factor over time: patients with LR without DM for 3 years remain at higher risk for development of DM compared to patients without LR (3 years cDMFS LR 91.8\% vs no-LR 99.6\%). Using the date of LR as "time zero", the risk of DM remains higher until patients achieve 5 years of follow-up when compared to patients without LR (5 years cDMFS LR 99\% vs no-LR 99\%). Conclusion Local regrowth is the only independent risk factor associated for development of distant metastases. The risk of distant metastases remains significantly higher for at least 5 years after the development of local regrowth. These results may suggest that development of local regrowth may determine a second "hit" in the risk of metastatic spread among patients who achieve a $\mathrm{cCR}$ and undergo organpreservation strategies with Watch and Wait.

\section{TOTAL LASER HEMORRHOIDOPLASTY: A NOVEL TECHNIQUE FOR ADVANCED HEMORRHOIDAL DISEASE}

\section{Alkiviadis Pappas Athens, George Bagias}

\section{Athens Medical Group, Athens, GR}

Background/Aim Laser hemorrhoidoplasty (LHP) is a new minimal invasive and painless procedure for day-surgery treatment of symptomatic hemorrhoids. To date, LHP is recommended for low-grade hemorrhoidal disease $\left(\mathrm{I}^{\circ}-\mathrm{II}^{\circ}\right)$, yet little has been known for the role of LHP in advanced hemorrhoidal disease with prolapse. In this study we examine the feasibility and efficacy of a modified LHP technique, the Total Laser Hemorrhoidoplasty in treatment of $\mathrm{III}^{\circ}-\mathrm{IV}^{\circ}$ hmorrhoidal disease. Methods We performed a retrospective analysis on consecutive patients operated for symptomatic 3rd- and 4th-grade hemorrhoidal disease from January 2015 to December 2017. Total Laser Hemorrhoidoplasty consists of two distinct phases; firstly, the hemorrhoidal nodules are lifted by lifting the mucosa in two or three spots, then a laser probe is driven between hemorrhoidal tissue and anal canal. Around $90-110 \mathrm{~J}$ of thermal energy are applied in each anal tertiary, not only at the level of hemorrhoidal nodules but throughout the anal canal. The procedure is done under deep sedation in day surgery. The patients are discharged usually 3 or $4 \mathrm{~h}$ after surgery. Results A total number of 581 patients were identified, with a mean age of 48.1 years $( \pm 13.6)$ and a male-to female ratio of $2.5: 1$. 322 patients $(55.4 \%)$ hade $\mathrm{III}^{\circ} \mathrm{HD}$ and 259 patients $(44.6 \%)$ had $\mathrm{IV}^{\circ}$ HD. During the first postoperative week 452 patients $(77.8 \%)$ used at least one analgesic; yet, the median VAS score was 5 (0-8). One month after surgery, only 32 patients $(5.5 \%)$ complained about pain, mostly by defecation; yet, the median VAS score was extremely low (1, 0-4). Regarding rectal bleeding, 64 patients (11\%) reported bleeding by defecation during the first postoperative week, with almost no episodes afterwards. All patients returned to their daily activities at 3rd postoperative day. Complications rate was $3.3 \%$ with local inflammation being the most prominent one, followed by abscess. No patients developed stricture or fissure. The mean follow up duration was 16.1 months. Nineteen patients (3.3\%) reported hemorrhoidal prolapse, and 8 of them sought medical attention. Total recurrence rate was $3.9 \%$ (23 patients), while 7 patients $(1.2 \%)$ were re-operated. Conclusion Total laser hemorrhoidoplasty is a fast and feasible technique for treatment of advanced hemorrhoidal disease. Postoperative discomfort as well as recurrence rates are considerably low; therefore it has the potential to evolve to the standard surgical procedure for treatment of $\mathrm{III}^{\circ}-\mathrm{IV}^{\circ}$ hemorrhoidal disease.

\section{SELECTIVE HISTOPATHOLOGICAL EXAMINATION FOLLOWING APPENDECTOMY IS ONCOLOGICALLY SAFE AND ASSOCIATED WITH SUBSTANTIAL COST SAVINGS}

Vivian P. Bastiaenen,Joske de Jonge, Bartholomeus J.G.A. Corten, Elise A.J. de Savornin Lohman, Anne C Kraima, Hilko A Swank, Jaap L.P. van Vliet, Gijs J.D. van Acker, Anna A.W. van Geloven, Klaas H. in't Hof, Lianne Koens, Philip R. de Reuver, Charles C. van Rossem, Gerrit D. Slooter, Pieter J. Tanis, Valeska Terpstra, Marcel G.W. Dijkgraaf, Willem Bemelman

Amsterdam UMC, University of Amsterdam, NL

Background/Aim Due to the increased workload of pathologists and significant costs, the necessity of routine histopathological examination following appendectomy has been questioned for several decades. Unfortunately, prospective studies investigating the oncological safety of a selective policy are lacking. Methods In this multicenter, prospective, cross-sectional study (ClinicalTrials.gov number: NCT03510923), all appendices removed for suspected appendicitis were macroscopically examined by the surgeon for abnormalities suspicious for neoplasms. Before sending all specimens for histopathological examination, the surgeon judged whether assessment by the pathologist was indicated. Main outcomes were the number of missed appendiceal neoplasms with clinical consequences benefiting the patient following a selective policy (upper limit twosided 95\% CI below 3:1000 considered oncologically safe) and 
potential cost savings. Secondary outcomes included clinical management and outcomes. Results Sixty-five (0.89\%) of 7339 specimens exhibited appendiceal neoplasms with clinical consequences. Following a selective policy, surgeons would have refrained $4966(67.7 \%)$ appendices from histopathological examination. Appendiceal neoplasms with clinical consequences would not have been diagnosed in 22 patients. Residual disease was radically removed in five patients, implying that an appendiceal neoplasm with clinical consequences benefiting the patient would have been missed in 1.01:1000 patients (upper limit 95\% CI 1.61:1000). Of the remaining seventeen patients, ten would have been saved from unnecessary surgery. Among 43 patients in whom appendiceal neoplasms with clinical consequences would have been diagnosed, thirteen benefited, while harm was observed in 27 patients. Estimated cost savings established by replacing routine for selective histopathological examination were $€ 703.500$ per 10.000 patients. Conclusion Selective histopathological examination following appendectomy is oncologicall y safe and will likely result in a reduction of pathologists' workload, costs, and unnecessary additional resections. In fact, as both routine and selective histopathological examination resulted in clinical consequences which were more often harmful than beneficial, even complete abandonment of histopathological examination following appendectomy might be justified.

\section{SYNCHRONOUS SQUAMOUS CELL CARCINOMA OF THE ANORECTUM AND RECTUM, A RARE COINCIDENCE}

\section{Rafaela Parreira, Joana Mendes, Miguel Almeida, Ana Faustino, Maria Inês Leite}

\section{Hospital Divino Espirito Santo de Ponta Delgada, Ilha Terceira, PT}

Background/Aim Primary squamous cell carcinoma (SCC) in the colorectum is rare and represent a small fraction of colorectal malignancies, since more than $90 \%$ of colorectal disease are adenocarcinoma tumors. Because of its rarity, very little information is available in literature about the etiology, prognosis and optimal treatment. Occurrence of SCC in synchronous form are even more rare and unique with only few cases reports published in literature. Methods Case report. Results A 68-year-old male patient present to emergency department complaining of asthenia and rectal bleeding for 6 months but with worsening in the last 15 days. He denied abdominal pain or changes in bowel habits. He had no associated comorbidities. At admission, the patient was alert, eupneic, pale tone, tachycardic $(123 \mathrm{bpm})$ and normotensive $(112 / 67 \mathrm{mmHg})$. Physical examination was normal, while digital rectal examination revealed presence of blood and a lesion with restricted mobility about $3 \mathrm{~cm}$ from the anal verge. Blood samples showed a severe anemia with hemoglobin $6.2 \mathrm{~g} / \mathrm{dL}$. Colonoscopy showed two lesions, a ulcerovegetative lesion above anal verge and extensive sessile lesion at middle/upper rectum. Both biopsies demonstrated a SCC. A contrastenhanced computed tomography of the chest, abdomen and pelvis was also done but no lesions were found in any other site than anus/ rectum. As for local staging, pelvis magnetic resonance imaging staging defined tumor as T4a, with mesorectum and suspected lymph nodes involvement and tumoral extension to anal sphincter. A PETCT scan was performed which was suggestive of metabolically active nodular lesion localized to anorectum and to rectum. After multidisciplinary discussion, the patient was proposed to neoadjuvant radiotherapy and quimiotherapy. The response to the systemic treatment was favorable and the patient was submitted to abdominoperineal resection. Conclusion The prognosis for squamous cell carcinoma of the colorectum is worse than for that of adenocarcinoma, mostly because of the delayed diagnosis. The etiopathogenesis of SCC is still unclear. Extensive evaluation needs to be done to rule out all sites of possible primary disease before confirming the diagnosis. The occurrence of squamous cell carcinoma of the rectum in concurrent with squamous cell carcinoma of the anal canal is a unique occurrence and there are only a few cases report in literature. The management of these patients should be discussed in multidisciplinary team.

\section{LAPAROSCOPIC MANAGEMENT OF TRANSMESOSIGMOID HERNIA: OUR EXPERIENCE AND SYSTEMATIC REVIEW OF LITERATURE}

Stefano Lafranceschina, Paolo Frattini, Antonio Modoni, Elena Ventrella, Gianfranco Malagnino, Massimo Buonfantino

Ospedale San Paolo, Bari, IT

Background/Aim Mesosigmoid hernias (SMH) are especially rare and account only for $6 \%$ of all internal hernias. Approach to SMH is still challenging due to non-specific clear clinical features and imaging, until frank occlusion or strangulation occurs. We present a rare case of 40-year-old female patient affected by SMH managed by laparoscopy and literature review. Methods A 40-year-old woman was admitted in emergency with persistent vomiting and leucocytosis. Anamnesis revealed a laparoscopic left oophorectomy for endometriosis. On physical examination she presented generalized abdominal distension with mild tenderness on palpation. CT scan demonstrated dilated small bowel and a closed loop of small bowel in the pelvis. Emergency laparoscopy revealed a mechanical small bowel obstruction due to an incarcerated internal hernia. A loop of an ileum had herniated through a full-thickness defect in the sigmoid mesocolon, consistent with a transmesosigmoid hernia. The defect was slightly enlarged and the herniated loop was successfully reduced. After the repositioning, incarcerated small bowel regained vitality, peristalsis and was therefore not resected. The mesosigmoid defect was repaired with four stitches. The patient had an uneventful postoperative course and was discharged on 9th postoperative day. Results The understanding of SMH by improving of diagnostic images have expanded: $72.7 \%$ of patients had intestinal necrosis and therefore intestinal resection was required. Although a conservative approach has been attempted, all patients undergone surgery. The strangulation of the herniated loops in the SMH defect is closely related to the size of the opening. The average diameter of the defect is approximately $3.58 \mathrm{~cm}$ (range $2-5 \mathrm{~cm}$ ). Conclusion When the defect diameter is $2-5 \mathrm{~cm}$, spontaneous resolution becomes unlikely and the risk of strangulation increases. Timely and periodic reassessment of the conditions of patients receiving conservative treatment is mandatory to allow early recognition of intestinal ischemia. In this regard, CT can also be used to re-evaluate intestinal vitality. Laparotomy is certainly a valid tool, although as demonstrated by our experience, laparoscopic management must also be encouraged in emergency, especially if resection is not necessary and intervention requires only defect reduction and herniorraphy with the resulting advantages: better short and long-term outcomes and less hospitalization time. 


\section{LONG-TERM OUTCOMES AFTER COLORECTAL SURGERY IN PATIENTS WITH ULCERATIVE COLITIS-ASSOCIATED COLORECTAL CANCER VERSUS SPORADIC COLORECTAL CANCER. A PROPENSITY SCORE MATCHED RETROSPECTIVE COHORT STUDY WITH SYSTEMATIC REVIEW AND META- ANALYSIS}

Viviane Lin, Robin Lohse, Michael Madsen, Tina Fransgaard, Feza Remzi, Ismail Gögenur

Center of Surgical Science, Kфge, DK

Background/Aim Ulcerative colitis is associated with a higher risk for developing colorectal cancer, however, it is unknown if this translates into a worse prognosis when malignancy occurs. The aim of this study was to compare long-term outcomes between patients with ulcerative colitis-associated colorectal cancer and sporadic colorectal cancer. Methods All patients who underwent surgery with curative intent for colorectal cancer in Denmark between 1st of January 2004 and 30th of June 2016 were included in the study. Patients diagnosed with ulcerative colitis and colorectal cancer were identified and matched 1:5 with patients with sporadic colorectal cancer using propensity score matching. The primary outcome was disease-free survival, with recurrence-free survival and all-cause mortality as secondary outcomes. In order to relate the results of the current study to the existing literature, a systematic review with meta-analysis was conducted. Results A total of 1332 patients, 222 with ulcerative colitis and 1110 with sporadic colorectal cancer were included in the study. Disease-free survival was similar between the two groups with a HR 1.06 (95\% CI 0.85-1.32), as was recurrence-free survival HR 1.14 (95\% CI 0.86-1.53) and all-cause mortality HR 1.15 (95\% CI $0.89-1.48)$. The results of the systematic review identified seven other relevant studies, whereof none reported on disease-free survival, one on recurrence-free survival, and seven on all-cause mortality. Metaanalysis including the results of the current study showed a HR 1.67 (95\% CI 0.61-4.56) for recurrence-free survival, and HR 1.21 (95\% CI 0.93-1.56) for all-cause mortality. Conclusion Based on the current study and the existing literature there were no significant differences in long-term outcomes between ulcerative colitis-associated and sporadic colorectal cancer. However, the current results are limited by possible residual confounding and the meta-analysis by heterogeneity in confounding adjustment.

\section{ONCOLOGICAL IMPACT OF COVID 19 IN THE CANAVESIAN REGION OF PIEDMONT}

\author{
Luca Panier Suffat, Lorenzo Bono, Ivan Lettini, Stefano Pileci, Guido \\ Mondini, Lodovico Rosato
}

ASL TO 4, Pont Canavese, Turin, IT

Background/Aim Coronavirus disease 2019 (COVID-19), first reported in Wuhan, China, in December 2019, among a group of individuals presenting with pneumonia of unknown ethiology, is the greatest public health crisis since the influenza pandemic of 1918 . This emergency needs a new evaluation and allocation of resouces. In the last months surgeons have postponed or cancelled many elective treatment, such as the screening programs, reserving admission to the hospital only to patients with malignancy which could pose a threat to survival. Methods In our surgical Department, hub for territorial diagnosis and treatment colorectal cancer, from January and 15
October 2020, we treated 63 patients for colorectal cancer and analized their characteristics. Results 21 (33\%) patients underwent surgery in urgency for an occlusive disease. Among these group we performed 16 Hartmann procedure (76\%), 2 concomitant bladder resection and 3 ileal resection because of extra colonic organ disease involvement. Retrospectively analyzing the oncological data we found a great prevalence of advanced diseases, with 44 patients $(69.8 \%)$ in an advanced stage (T3 or more or $\mathrm{N}+$ ). Conclusion Careful assessment of risk and benefit is always required, but in the pandemic period the risk for contamination should be considered. Visits from relatives are not allowed in the hospital, so the patients undergoing major surgery should experience a significant isolation time with inevitable negative psychological consequences. In this scenario surgeon must accept compromises choosing a treatment with low short term complication rates and requiring less organizational efforts to reduce possibilities of contamination and pulmonary complications. Medical and surgical complications in oncological patients will be more carefully evaluated, including a diffuse analysis and definition of quality of life for patients and their families. The possibility of prolonging hospital stay, including significant time in the Intensive Care Unit, will be at the center of the surgical evaluation, for an adjusted risk-benefit balance sheet.

\section{PROPHYLACTIC PARASTOMAL MESH PLACEMENT FOR END COLOSTOMY AND ITS IMPACT ON PARASTOMAL HERNIA FORMATION}

Patrick Jull, Kelvin Adasonla, Rita Joarder, Andrew Aldridge, Raj Harshen, Giuseppe Preziosi

\section{East Sussex Healthcare NHS Trust, St. Leonards on Sea, GB}

Background/Aim Parastomal hernia (PSH) is common following colostomy formation. While the role of prophylactic mesh placement at the time of permanent end colostomy formation is debated, we aimed to assess whether a mesh has an impact on the risk as well as size of any hernia formed. Methods We collected data for consecutive patients undergoing elective permanent end-colostomy formation between 2013 and 2019, who had follow-up imaging. The study group was made of patients who had a prophylactic biological porcine mesh routinely implanted, with a sub-lay technique, at the time of permanent end-colostomy formation. This was compared to a contemporaneous non-mesh control group. CT scans were reviewed by a gastrointestinal radiologist, blinded to cohort and clinical outcomes, to assess for the radiological presence and grade of PSH according to the classification adopted by the British Hernia Society: IA: Bowel forming the colostomy with a sac $<5 \mathrm{~cm}$ IB: Bowel forming the colostomy with a sac $>5 \mathrm{~cm}$ II: Sac containing omentum III: Intestinal loop other than the bowel forming the stoma. Results Seventy-one patients identified who did not have prophylactic mesh placement compared to 70 who had. After excluding those who did not have imaging, 68 patients remained in the non-mesh group and 60 in the mesh group. There was no significant difference in baseline characteristics between the two groups, including BMI $[p=0.425]$, age $[p=0.478]$ and colorectal cancer diagnosis $[p=0.156]$. However the mesh group had longer follow-up [32 (20.1) vs 42 (22.8) months, $\mathrm{p}<0.05]$. Whilst there was no difference in the incidence of radiologically diagnosed PSH [non-mesh $48(71 \%)$ vs mesh 39 (65\%), $p=0.498]$, a higher proportion of type III hernias was observed in the no-mesh cohort [non-mesh $17(35 \%)$ vs mesh $3(8 \%), \mathrm{p}<0.05]$. No difference between groups was observed for the other hernia grades. Conclusion Prophylactic insertion of a parastomal mesh at the time of end colostomy formation might reduce the chance of a grade III 
parastomal hernia. This is clinically significant, as these hernias are more likely to be symptomatic or become obstructed.

\section{COLONIC MALT LYMPHOMA: AN UNUSUAL DIAGNOSE FROM A REGULAR OBSTRUCTION}

\author{
Tania Valente, Sandra Amado, Ines Goncalves, Nuno Ferreira, \\ Goncalo Ferreira, Arnaldo Figueiredo, Miguel Coelho

\section{Centro Hospitalar de Leiria, Leiria, PT}

Background/Aim MALT lymphoma represents around 7-9\% of nonHodgkin lymphomas. Frequently affecting the stomach, it can also be found in salivary glands, thyroid, skin and lung, among others. The colon is the rarest location for gastrointestinal tract MALT lymphoma, accounting for only $0.2 \%$ of colorectal cancers. Usually found incidentally, it can present with abdominal pain, constipation and weigh loss. Colonoscopy findings vary from single or multiple polypoid lesions and/or ulcerations. So far, there isn't a consensual treatment strategy. However, the prognosis is overall excellent, with a 5 -year disease free survival of $92 \%$. Methods Retrospective descriptive study of a case report based on information from clinical records, patient observation and analysis of complementary exams. Results We present a case of a 73-year-old male admitted at the emergency department with obstipation for 3 days. He denied vomiting and the abdomen was tender. There were no feces or blood on digital rectal examination and the CT-scan showed a $54 \mathrm{~mm}$ lesion in the descending colon, with extra-parietal involvement, regarding an exofitic tumor or adenopathic conglomerate. A left hemicolectomy was performed and the postoperative course was uneventful. Histological examination revealed a MALT lymphoma. Staging CT showed no evidence of metastatic disease and the gastric biopsies were negative for $\mathrm{H}$. Pylori. A Hematology consult was requested for further testing and treatment. Conclusion Colonic MALT lymphoma is rare, with very few published cases. In our case report, the patient presented with obstipation, leading to surgery as the first therapeutic approach. More cases will be needed to determine the best treatment and define a standard of care.

\section{PERFORATED JEJUNAL DIVERTICULUM-A RARE ENTITY}

\section{Rita Martelo, João Morais, Maria Angeles Rabago, Cláudia Marques, Francisco Rodrigue}

\section{Hospital Vila Franca de Xira, Lisbon, PT}

Background/Aim A jejunal diverticulum is a rare condition that affects only $1 \%$ of the general population, with a slightly higher prevalence in men. Perforation is a known complication but it is an unusual cause of acute abdomen and is difficult to diagnose since it may mimic the symptoms of a variety of diseases. Methods We report the case of a 35-year-old male, with a known diagnosis of Gilbert's syndrome, who presented to our emergency department with a 3-day history of fever, peri-umbilical abdominal pain and jaundice. Analyses revealed leukocytosis, an elevation of CRP and elevation of total bilirubin at the expense of indirect bilirubin. CT scan revealed a heterogeneous mass in the mesenteric root, with $5 \mathrm{~cm}$, containing extra luminal air and a calcified object adjacent to a small intestine loop, that could be related to an endoluminal foreign body with an contiguous collection or a diverticular mass with an associated inflammatory process. Results We decided to perform an urgent laparotomy, since there was considerable distention of the small intestine. Intra-operatively we found an abscess surrounding a jejunal diverticulum, located in the mesenteric border, measuring $8 \times 4 \mathrm{~cm}$, $70 \mathrm{~cm}$ distal to the ligament of Treitz, with an inflammatory mass at its tip, probably due to a foreign body reaction after partial perforation. Since there was considerably inflammation of the adjacent intestinal loop, we decided to perform an enterectomy with primary mechanical anastomosis. The surgical specimen confirmed a jejunal diverticulum perforated by an unidentified green object. The patient remembered that he had chewed a beer cap several days ago. He was discharge free of complications 5 days after the surgery. Conclusion Almost $50 \%$ of the jejunal diverticula are asymptomatic. Perforation is a rare complication and peritonitis can be localized because the diverticula are on the mesenteric border of the bowel and allow the small bowel mesentery to wall them off. The treatment of choice is segmental intestinal resection with primary anastomosis, since diverticulectomy may impair blood flow because of its mesenteric location, leading to anastomotic breakdown or fistula formation.

\section{A SINGLE SITE EXPERIENCE OF POST- COLONOSCOPY COLORECTAL CANCERS: IS THERE A MISSED LINK FOR THESE PATIENTS?}

Amandeep Dosanjh, Pradeep Janardhanan, Iram Parwaiz, Paul Lim, Shuker Yahia, Aravindan Narayanan

\section{Solihull, GB}

Background/Aim Colorectal cancer (CRC) is the 4th most prevalent cancer in the UK and accounts for around 45 deaths per day nationwide. The gold standard diagnostic modality is colonoscopy with histology; this is however dependant on a number of factors. There are a small number of patients that are diagnosed with CRC following a colonoscopy reported as normal, defined as a post-colonoscopy colorectal cancer (PCCRC). The Joint Advisory Group on GI endoscopy (JAG) recommended a quality indicator of $<5 \%$ PCCRC. This study aims to examine all colorectal cancers George Eliot Hospital (GEH) over 4 years and review common factors for PCCRC. Methods This is a single site retrospective study aggregating the result of 3 audits with identical methodology; Mar 15-Mar 17, Mar 18-Mar 19, and Apr 19-Mar 20. All patients diagnosed with colorectal cancer were identified from the hospital records. The electronic health record and unisoft databases were manually interrogated to identify patients with PCCRC. The definition was diagnosis of CRC within 36 months of a colonoscopy reported as normal—or for left sided lesions a normal flexible sigmoidoscopy. Records of those with PCCRC were examined in closer detail. Results 449 patients were identified with CRC in total. The majority of patients $(52.8 \%)$ were diagnosed by colonoscopy, $22.3 \%$ were diagnosed on CT scan, $12.5 \%$ were diagnosed by flexible sigmoidoscopy, $7.8 \%$ by CT-colonography and $4.7 \%$ by other modalities. In total there were 6 patients that met the criteria for PCCRC. Of these patients $50 \%$ had a tumour in the ascending colon, $16.7 \%$ in the caecum and $33.3 \%$ were rectosigmoid. All cancers were $\mathrm{T} 2$ or above. There was no pattern to indication for endoscopy, 2 patients had inflammatory bowel disease screening scopes and the rest had 2 week wait criteria. Half of endoscopies were consultant performed and half by specialist nurse. There were no procedural complications and discomfort was only noted for one patient. Prep quality was documented for $50 \%$ of PCCRC, 2 patients had poor prep and 1 moderate. Withdrawal time was documented for 1 patient. Conclusion There is an extremely low rate of PCCRC at George Eliot Hospital, 1.3\% of all cancers diagnosed had a previously normal endoscopy within 3 years. There was no common theme identified for patients with PCCRC, with the caveat that PCCRC rates were low and provided a small 
sample size. We would recommend a national database study of all PCCRC, utilising the JAG database, in order to identify those patients at risk of PCCRC.

\section{TRENDS IN INCIDENCE OF YOUNG ONSET COLORECTAL CANCER IN SCOTLAND}

\author{
Viswan Thiagarajah, James Kynaston
}

\section{Dunblane, $G B$}

Background/Aim Studies have reported a rising incidence of colorectal cancer in young adults across the globe. The aim of this study was to review incidence of young onset colorectal cancer in Scotland over a 24 year period. Methods An observational study was carried out to examine the incidence rates of colorectal cancer in Scotland between January 1994 and December 2018. Patients aged between 20 and 49 were included and divided into three age groups (20-29, 30-39 and 40-49). Incidence rates were extracted from the Scottish Heath and Social care platform under the following ICD 10 codes: C18, C19-20, C18-20. Joinpoint regression software was used to analyse temporal trends in the log-transformed incidence rates. Results Across both proximal and distal colorectal tumours, the greatest rise in incidence rates was observed in those aged 20-29. For distal tumours in those aged 30-39 there was a continuous increase over the 24-year study period. In contrast, the incidence rates of proximal and distal tumours remained stable in those aged 40-49. Sub-analysis by gender revealed no significant difference in incidence rates between males and females. Conclusion There has been a significant rise in incidence rates of colorectal cancer in Scotland in those aged 20-39. The largest increase was in the 20-29 age group. Awareness of this may help inform both the public and medical professionals that colorectal cancer can affect the young as well as the old.

\section{COMPARISON AMONG THREE PREPARATION TECHNIQUES FOR COLONOSCOPY. ANALYSIS OF 300 CASES}

\section{Monica De Jesús, Quintín H Gonzalez, Jesus A Bahena}

\section{Hospital HMG Coyoacán, Mexico, MX}

Background/Aim To compare three preparation techniques used in colonoscopy in terms of tolerance, side effects, and efficacy of colon cleansing. Methods A prospective, randomized and comparative study was carried out in which a sample of 300 cases was analyzed from 2015 to 2020, which were randomly distributed into three groups, assigning each group a different preparation. Group A was given sodium phosphate (fleet fosfosoda), which was administered orally, two $45 \mathrm{ml}$ bottles diluted in $250 \mathrm{cc}$ of water each at 18 and $20 \mathrm{~h}$, group B was prepared with two picosulfate sachets sodium (picoprep) at 18 and $20 \mathrm{~h}$ diluted in a glass of $250 \mathrm{cc}$ each and between one and the other 3 glasses of $250 \mathrm{cc}$ of water. Group $\mathrm{C}$ were administered two bisacodyl tablets in a single oral dose and two macrogol sachets (NULYTELY bis) diluted in $1 \mathrm{~L}$ of water each taken at 19 and $21 \mathrm{~h}$ and a liquid diet the day before the study. Results For all patients the Boston scale was used to evaluate the preparation, group A obtained an average on the scale of 8 , in group B it was obtained 9 and in group $\mathrm{C}$ a score of 8 , without observing a difference statistically significant with a $\mathrm{p}$ value $>0.05$. Regarding the tolerance of the preparation, in group A it was reported that $23 \%$ referred nausea, $31 \%$ referred colicky abdominal pain, $5 \%$ presented vomiting and a level of mild dehydration. In group B $30 \%$ had nausea,
$37 \%$ abdominal pain, $13 \%$ vomiting and the level of dehydration was mild and in group C $33 \%$ reported nausea, 36\% presented vomiting, $18 \%$ reported abdominal pain and the level of dehydration was moderate, it should be noted that in this last preparation the most important symptom was vomiting, so the tolerance level of the patients was higher for preparation $\mathrm{A}$ and $\mathrm{B}$, however they show a value of $p>0.05$. Complications were reported in no patient during colonoscopy. Conclusion The three preparations are effective for the preparation with the Boston scale without reaching a statistically significant difference and in terms of side effects, greater effects were observed in the preparation of group C (Nulytely bis) due to the volume that the patient must take in a short time, however when comparing them they did not reach a statistically significant difference.

\section{MANAGEMENT OF ACUTE DIVERTICULITIS IN HOSPITAL SETTING: A SINGLE CENTER RETROSPECTIVE STUDY}

Catarina Osório, Luísa Frutuoso, Joana Correia, António Soares, Rosa Sousa, Jorge Costa, Mário Nora

CHEDV_Centro Hospitalar de Entre o Douro e Vouga, Vila Nova de Gaia, PT

Background/Aim Acute diverticulitis (AD) is one of the most common gastrointestinal disorders among inpatients. The majority of admitted patients with $\mathrm{AD}$ can be managed medically and emergent surgical treatment is required in only $15-25 \%$. The morbidity and mortality for emergency surgery in AD has been associated with rates has high as $25 \%$ and $50 \%$, respectively. Our objective was to study the inpatient management of $\mathrm{AD}$ in our surgical department and compare it to the current practice. Methods A retrospective review of patients admitted in the general surgery department of a Portuguese secondary hospital, from July 2017 to June 2020 for acute diverticulitis. We analyzed demographic data, the severity of the disease (according Modified Hinchey classification), length of stay, surgical and medical treatment, morbi-mortality and follow-up. Results A total of 94 patients (46 men and 48 women) with $\mathrm{AD}$ diagnosis were admitted in our department. The mean age was $62.3 \pm 14.7$ years old. $43 \%$ of patients had previous diverticulosis and in $19 \%$ the present episode was not the first. $60 \%$ of the admitted patients had complicated AD at admission (? Hinchey Ib). Hinchey Ib and II was present in $38 \%$ of patients; treatment was managed by percutaneous drainage in $14 \%$ of the cases and in $19 \%$ surgery was needed. Among the surgical cases, $70 \%$ had intraoperative Hinchey III AD independently of the Hinchey classification found at admission. Peritoneal toilet with drainage was the most used surgical technique $(40 \%)$, followed by Hartmann procedure (15\%). $25 \%$ of the procedures were performed by laparoscopy. The overall postoperative morbidity and mortality was $40 \%$ and $20 \%$, respectively. Mean length of hospital stay was $7.3 \pm 5.8$ days. In $2 \%$ of patients a colorectal cancer was diagnosed in the follow-up colonoscopy and $9.6 \%$ of the patients required posterior elective resection ( $89 \%$ performed by laparoscopy) for persistent symptoms or colorectal cancer. Conclusion In conclusion, the majority of patients admitted for $\mathrm{AD}$ can be managed medically or with minimally invasive procedures. Emergent surgical treatment should be reserved for patients with diverticular peritonitis or who fail to respond to medical treatment. When necessary, surgeons should favor minimally invasive surgical techniques and avoid a permanent stoma, to improve patient's quality-of-life and reduce overall morbidity and mortality. 


\section{CONSIDERATIONS OF MORTALITY AND FUNCTIONAL STATUS IN COLORECTAL CANCER SURGERY IN THE ELDERLY}

\author{
Samantha Greenfield, Victoria Russell, Paul Ziprin \\ Imperial College, London, GB
}

Background/Aim Colorectal cancer surgery in elderly patients is inherently high-risk due to patients' lack of functional reserve and comorbidities. 30-day postoperative surgical mortality is a well-defined parameter of survival in colorectal surgery, but longer survival outcomes are less well-reported. In this study, we analysed the outcomes of a large cohort of patients undergoing major colorectal cancer resection. We compared 30-day, 90-day and 1-year mortality in $<75$ and $>75$ patient groups, as well as investigating the change in their performance status, performance score, and length of stay (LOS). Methods Patients undergoing colorectal cancer resection between December 2017 and October 2020 were prospectively identified from a Teaching Hospital single-centre cancer database. Patients were stratified into $>75$ years and $<75$ years groups. Primary outcome measures were postoperative 30-day, 90-day, and 1 -year mortality. Secondary outcomes were change in Montreal Frailty Score and Performance Score with respect to pre-morbid status and discharge status, and LOS. Results We examined 1903 records for patients referred on the Trust Colorectal Cancer Pathway between October 2017 and April 2020. 342 patients underwent surgery, $<75$ $\mathrm{n}=257,>75 \mathrm{n}=82.1$ patient died in the $<75$ group at 30 days $(0.39 \%)$ compared to $\mathrm{n}=3(3.7 \%)$ in the $>75$ group $(\mathrm{p}=0.019)$. Mortality at $90 \mathrm{~d}$ is not statistically significant with $\mathrm{p}=0.109 .14 / 196$ patients died at 1 year in the $<75$, compared to $14 / 61$ in the $<75$ group $(\mathrm{p}=0.003)$. We found a statistically significant decrease in the performance and frailty scores in the elderly group (frailty $p=0.000021$, performance $p=0.0003$ ), and a significantly higher LOS $(p=0.018)$. Conclusion The findings suggest though there is no statistical difference in mortality at $90 \mathrm{~d}$ between the two age groups, there was a markedly lower survival at 1 year. We found significantly increased LOS and marked worsening of performance and frailty scores in elderly patients. This equates to increased burden on posthospital community support. This study suggests that the decision to operate on elderly patients with colorectal cancer needs to be weighed carefully with the potential for significant reduction in survival, performance, and quality of life. This should be reflected in communication between patients and their relatives prior to operative intervention.

\section{USING INDOCYANINE GREEN (ICG) DURING LAPAROSCOPIC BOWEL RESECTIONS FOR ELECTIVE CANCER PATIENTS REDUCES ANASTOMOTIC LEAKS AND INPATIENT LENGTH OF STAY}

\section{Anna Bleakley, Olusegun Komolafe \\ NHS Lanarkshire, Glasgow, GB}

Background/Aim Anastomotic leakage is the dreaded complication in colorectal surgery, associated with significant morbidity and mortality. Poor perfusion of the bowel anastomosis is a significant contributing factor. ICG is a dye administered intravenously during laparoscopic colorectal surgery to assess bowel perfusion by fluorescent imaging. Our hospital was the first in our region to routinely use ICG and the aim of this study was to determine whether its use has led to improved patient outcomes after elective colorectal laparoscopic resections for cancer. Methods This is a single-centre comparative study of a prospectively maintained database of 75 consecutive patients who underwent elective colorectal laparoscopic resections for cancer from January 2019 until October 2020, with clinical data collected from electronic notes. The primary outcome investigated was anastomotic leakage. Secondary outcomes investigated included clinical suspicion of anastomotic leak (patients who went on to have a CT abdomen and pelvis for suspicion of anastomotic leakage), post-operative ileus, and in-patient length of stay. Cohorts were compared with 2 test. Results 19 patients had resection with ICG fluorescence angiography, and 56 patients without. No patient in the ICG group, and three patients in the non-ICG group (5.4\%) had anastomotic leakage; p-value 0.31 . The ICG group were less likely to have CT for suspected anastomotic leak $10.5 \%(2 / 19)$ vs $23.2 \%$ (13/56), p-value 0.29; and, post-operative ileus 5.3\% (1/19) vs $19.6 \%$ (11/56), p-value 0.18 . There was statistically significant reduction in mean inpatient length of stay when ICG was used (3.9 days, 95\% CI 3.2-4.7) compared to when it was not used (6.8 days, 95\% CI 5.0-8.5). Conclusion Only a small number of previous studies have compared anastomotic leak rates with and without ICG and they have found that its use leads to a significant reduction in anastomotic leakage. Our sample size, though small, supports these findings. We also found that using ICG led to a significant decrease in inpatient length of stay, likely reflecting the morbidity associated with anastomotic leakage. ICG fluorescence angiography is now established as our normal practice for all colorectal resections. It is a safe, innovative technology easy to incorporate in to existing surgical practice.

\section{PREVALENCE OF GASTROINTESTINAL SYMPTOMS IN ACUTE EMERGENCY PATIENTS-WHAT THE COLORECTAL SURGEON NEEDS TO KNOW}

Kristof Nemeth, Rahman Shahinur, Melissa Ong, Christiana Boules, Mohamed Toeima, Ioannis Nikolopoulos

\section{Bromley, $G B$}

Background/Aim Gastrointestinal (GI) symptoms have been reported with coronavirus disease (COVID-19), but data on the prevalence and the association with severity of disease are limited. This can be a safety concern for surgeons as they might see COVID-19 patients presenting with primary GI symptoms. We sought to characterise the GI manifestations of COVID-19 in acute patients attending the emergency department. Methods We performed a review of our emergency departments prospectively maintained database of patients between 20/03/2020 and 20/04/2020 who had positive Polymerase Chain Reaction (PCR) COVID-19 test. We evaluated the prevalence of GI symptoms and their association with the severity of COVID-19. We also looked at the ethnicity of patients and its possible role in the prevalence of symptoms. Chi-squared test of the R statistical software was used to analyse the data. For the analysis of Chi-squared test of independence between multiple groups the "rcompanion" package of the same software environment was applied. Results Of the 174 eligible patients (94 male) 12 had nausea, 14 vomiting, 37 diarrhoea and 9 abdominal pain. 53 patients were very frail and early discussion about resuscitation status was carried out following which they received active treatment on ward level with a do not resuscitate order being in place. Of the other 121 patients, 16 were admitted to ITU and 9 patients died ( 7 of these patients were admitted to ITU). There was no demonstrable difference for nausea $1 / 11$ severe, $P=0.9$ ), vomiting $(0 / 11$ severe $)$, diarrhoea $(6 / 28$ severe, $\mathrm{P}=0.39)$ and abdominal pain $(1 / 7$ severe $\mathrm{P}=1)$ between the severe and non-severe groups. We 
were also unable to demonstrate any difference between different ethnic groups (29 Black patients, 12 Asian patients, 54 White patients and 24 Other Ethnicities). Nausea (4 Black, 0 Asian, 7 White and 0 Other ethnicities, $\mathrm{P}=0.15$ ), vomiting ( 3 Black, 2 Asian, 5 White, 1 Other ethnicities, $\mathrm{P}=0.67)$, diarrhoea (10 Black, 2 Asian, 9 White and 7 Other ethnicities $\mathrm{P}=0.25)$ and abdominal pain $(1$ Black, 2 Asian, 1 Other ethnicities, $\mathrm{P}=0.49$ ). Conclusion Acute GI symptoms associated with COVID-19 are highly prevalent in emergency patients and the attending surgeon should consider COVID-19 infection in all patients presenting with GI symptoms even without respiratory symptoms. We have not been able to demonstrate increased prevalence of GI symptoms in severe cases and there was no statistical difference in the prevalence of GI symptoms in different ethnic groups.

\section{THE RETROPERITONEAL FASCIA-A ROADMAP TO A SAFE COMPLETE MESOCOLIC EXCISION}

\author{
Tushar Mishra, Ankit Sahoo, Pankaj Kumar, Prakash Kumar Sasmal
}

AIIMS Bhubaneswar, Bhubaneswar, IN

Background/Aim Adequate knowledge and understanding of the retroperitoneal anatomy is important not only for complete mesocolic excision, but also to improve the safety of the surgery. The retroperitoneal fascia remains key to direct the steps of the surgery as well as decrease chances of injury to important structures like the ureter and the duodenum. We aim to outline how the retroperitoneal fascia guide the surgery. Methods We routinely perform laparoscopic radical right hemicolectomy by the medial to lateral approach creating a retroperitoneal tunnel. We present our understanding of the retroperitoneal fascia and how It can be used as a road map for a standardizing the technique for complete mesocolic excision for rightsided colonic carcinoma. Results We find the fascial plane a useful landmark to protects the ureter, duodenum and the pancreas. Conclusion The impact of the fascial anatomy on the operative steps will be emphasized which becomes all the more important, when the surgery is done laparoscopically.

\section{SUBCUTANEOUS LEIOMYOSARCOMA: A 5-YEAR CASE SERIES OF AN ONCOLOGY INSTITUTE IN PORTUGAL}

Mariana Claro, Pedro Martins, Paula Pinto, Inês Mónica, Augusto Moreira, Joaquim Abreu de Sousa

Hospital do Litoral Alentejano, E.P.E., Santarém, PT

Background/Aim Skin leiomyosarcomas are rare malignant neoplasms that derive from smooth muscle tissue, accounting for 2-3\% of all soft-tissue sarcomas. Subcutaneous leiomyosarcomas have a poor prognosis, with high rates of local-regional recurrence and metastasis (30-40\%). We present a systematic review of all patients with a histopathologic diagnosis of subcutaneous leiomyosarcoma in Instituto Português de Oncologia do Porto between 2013 and 2017. Methods We present 2 different cases of subcutaneous leiomyosarcomas. Results The first case reports to a 62-year-old male presenting with a left peri-anal nodule with a 2 months growth. Excisional biopsy performed in March 2013 was compatible with a $3.5 \mathrm{~cm}$ intermediate grade leiomyosarcoma with a mitotic index $>20 \mathrm{mi}$ toses/HPF and tumor necrosis; with deep margins involved. He was then sent to our Institution and proposed to a wide excision with a Limberg Plasty. Thoraco-abdominopelvic CT and pelvic MRI showed no distant metastasis or residual disease. The procedure went uneventfully and no residual disease was found on the histopathology. At 1-year post op, the patient developed a $4 \mathrm{~cm}$ inguinal mass compatible with lymph node metastasis. PET scan also revealed lung metastasis. The patient was started on palliative chemotherapy with doxorubicin with poor response and rapid disease progression and died 2 years after. The second case is of a 61-year-old female with a history of peri-anal tumefaction with a 1-year evolution. The excisional biopsy taken on July 2013 showed a $1.5 \mathrm{~cm}$ low grade leiomyosarcoma with a mitotic index of 8 mitoses/HPF without tumor necrosis. The patient underwent a wide excision of the scar without evidence of residual disease. Upon referral to our Institution, active surveillance was decided after discussion on a multidisciplinary team. The patient remains disease free until the present date. Conclusion Surgical treatment is the gold standard for localized tumors and of paramount importance on local disease control. However, distance recurrence is much more common as tumor biology remains the main survival free predictor. Hence, surgical functional outcomes must be heightened.

These two cases suggest that are the tumors' characteristics rather than the quality of the surgery that dictates patient's prognosis. The role of radiotherapy has not been clearly defined.

\section{ADVANTAGE OF INTRAOPERATIVE ULTRASOUND SCAN FOR IDENTIFICATION OF SMV IN OBESE PATIENTS UNDERGOING ROBOTIC CME FOR RIGHT SIDED COLON CANCER}

Vicky Maertens, Samuel Stefan, Ioannis Mykoniatis, Najaf Siddiqi, Mira Wagh, Jim Khan

\section{Birdham, GB}

Background/Aim In patients with right sided colon cancer Complete Mesocolic Excision (CME) appears to have some oncological benefits compared to conventional colectomy. The CME is however a more complex operation due to variability in vascular anatomy and the associated potential morbidity such as SMV injury. Identification of SMV can be facilitated especially in obese patients with the novel use of intraoperative ultrasound scan as reported by us previously. This study aimed to assess postoperative outcomes after robotic CME, comparing patients with BMI of 28 or less and obese patients with BMI 29 or more. Methods Seventy-five consecutive patients with right sided colon cancer underwent robotic CME using the Da Vinci $\mathrm{Xi}^{\circledR}$ system between December 2014 and December 2017. Patients were divided in to two groups, group 1 (non obese BMI $<28$ ) and group 2 (Obese BMI > 29). In group 2 patients, SMV detection was aided using the robotic ultrasound device. Patient demographics, operative findings and postoperative outcomes were evaluated, such as conversion rate, operation time, length of stay (LOS), complication rate and readmissions. Results Thirty-three (group 2, mean age 64.5 years) were compared with 42 patients (group 1 mean age 68 years). There were no significant differences in patient demographics between the two groups. There were no conversions in either group. The use of the intraoperative ultrasound scan did not impact on the operating time (median operative time of $180 \mathrm{vs} .185 \mathrm{~min}$ in group 1 and 2, respectively). Overall complication rate was not significantly different in both groups with $7(16 \%)$ in the group 1 vs. 6 (18\%) complications in the group 2). There were no cases of major vascular injury. One patient in group 2 had a lymphatic leak casing a lymphocele managed conservatively. One patient had anastomotic leak in group 2. There were no perioperative deaths. Median LOS was 6 vs. 5 days between the two groups and a similar number of patients 
were readmitted within 30 days ( 2 vs. 4 in group 1 and 2). R0 resection was achieved in $97 \%$ and $94 \%$ in the two groups with a median lymph node harvest of 33 and 31, respectively. Conclusion CME is technically more challenging than conventional colectomy and involves more risk, especially in obese patients. This study shows that results after robotic CME in obese patients are comparable to those of non-obese patients. Risk of vascular injury can be minimised with the use of intraoperative ultrasound scan.

\section{A CASE OF METASTATIC INVASIVE DUCTAL CARCINOMA TO COLON 27 YEARS AFTER INITIAL DIAGNOSIS}

\author{
Anzel Jansen Van Rensburg, Andrew Riddell
}

\section{Queensland Health, Redcliffe, AU}

Background/Aim Breast cancer is the most common neoplasm to affect women, affecting up to $32 \%$. Common sites of metastasis include liver, lungs and bone. Metastasis to the gastrointestinal tract is a rare. We describe a case of a 74 yo female that presents with metastatic breast cancer to bowel 27 years after initial diagnosis. A large case series reports breast cancer Gastrointestinal (GI) metastasis to be less than $1 \%$. Of these, $8 \%$ found in oesophagus, $28 \%$ in stomach, $19 \%$ to small bowel and $45 \%$ at the colon and rectum. Timing of diagnosis varies from 10 to 20 years. Most GI metastases are thought to be from lobular carcinoma despite ductal carcinoma being more common. The infiltration pattern seen in bowel is more diffuse for lobular whereas ductal appears more nodular. Methods 74 yo female initially diagnosed with invasive ductal carcinoma in 1991. She proceeded to wide local excision and axillary lymph node clearance. Post operatively she proceeded to radiation and chemotherapy. In 1996 she was diagnosed with bony metastasis and treated with Tamoxifen until 2001. Surveillance imaging found progression of ovarian lesions and patient proceeded to hysterectomy and bilateral salpingectomy. Histology confirmed metastatic ductal breast cancer. Her treatment was then changed to Aromasin until 2013 then ceased and she continued clinical follow up. Surveillance imaging then detected a retroperitoneal mass in July 2016 and patient restarted Aromasin. Surveillance colonoscopy in 2018 identified a fungating non obstructing mass in sigmoid colon. Histology from biopsy showed poorly differentiated invasive ductal carcinoma of breast. She proceeded to laparoscopic assisted anterior resection. Sigmoid colon showed metastatic invasive ductal carcinoma of breast with lymphovascular and perineural invasion. Fourteen of the seventeen lymph nodes showed metastatic breast cancer with extra nodal extension. Markers showed oestrogen positive, progesterone and HER2 negative. The patient remained on Aromasin therapy. Repeat colonoscopy in July 2018 showed no colon recurrence.

\section{Results/Conclusion}

Breast cancer metastasis to colon is very uncommon. It can be an incidental finding on surveillance investigations, however can also present with various radiological and clinical findings. This highlights the importance of considering this as differential in patients with history of previous breast cancer.

\section{MULTICYSTIC PERITONEAL MESOTHELIOMA (MCPM) OF THE ASCENDING COLON PRESENTING WITH ACUTE ABDOMINAL PAIN}

Mark Redden, Andrew Riddell
Redcliffe Hospital, Queensland Health, Redcliffe, AU

Background/Aim MCPM is a rare neoplasm arising from the mesothelial cells of the peritoneum. The pathophysiology is unclear but it most commonly occurs in young women and there is an association with endometriosis and previous surgery. Approximately 150 cases have been reported globally. MCPM usually presents with subacute abdominal pain or as an incidental finding and usually involves the pelvic peritoneum. Complete excision is the mainstay of therapy but local recurrence is common. Cytoreductive surgery combined with hyperthermic intra-peritoneal chemotherapy (HIPEC) may be effective in such cases. 1 death has been reported in a patient who declined further treatment for recurrence. We present a case of MCPM overlying the ascending colon which presented with acute abdominal pain. Methods A 32-year-old female presented to the emergency department with 1 day of right lower quadrant abdominal pain. She had a history of endometriosis, cholecystectomy and hysterectomy. Vital signs were normal and there was right side abdominal tenderness. Inflammatory markers were normal. Ultrasound abdomen did not identify the appendix or the right ovary but noted a $102 \times 43 \times 52 \mathrm{~mm}$ complex multilocular cyst. CT abdomen showed a low-density mass with adjacent inflammatory change anterior to the ascending colon. Given the inflammatory changes on imaging it was thought that the lesion represented an abscess. A drain was inserted under ultrasound guidance. Clear fluid was aspirated from the lesion and sent for analysis. M/C/S showed no growth. Cytology did not identify malignant cells. CEA and CA19.9 were negative. Results The patient's symptoms improved and she was discharged. A subsequent CT abdomen one month later showed that the lesion persisted. The patient then proceeded to laparoscopy which revealed a multi-cystic structure overlying the distal ascending colon and hepatic flexure. The lesion was adherent to the epiploic appendages and in places was continuous with the omentum. It was dissected and removed whole. Histology demonstrated MCPM. Conclusion This case of MCPM is unique due to the acuity of the presenting pain and the location of the lesion. It is important to consider MCPM as a differential in cases of intra-peritoneal cystic lesions. Complete excision is vital as local recurrence is common, however, repeat excision combined with HIPEC may be effective in cases of recurrence.

\section{PERIANAL ALVEOLAR RHABDOMYOSARCOMA: A CASE-REPORT}

Paula Pinto, Pedro Martins, Mariana Claro, Rita Canotilho, Ana Margarida Correia, Catarina Baía, Inês Mónica, Mariana Claro, Joaquim Abreu de Sousa

Instituto Português de Oncologia do Porto, Porto, PT

Background/Aim Alveolar rhabdomyosarcoma (aRMS) is the most undifferentiated subtype of rhabdomyosarcoma associated with an aggressive behavior, with a peak incidence at 10 to 25 years. aRMS most often arise in the extremities and perineal region although any location can be affected. $90 \%$ of aRMS are associated with recurrent Forkhead box O1 (FOXO1). The outcome of patients with perianal or perineal aRMS has been regarded poor with combined survival from 25 to $50 \%$. Treatment options have included chemotherapy, conservative or radical excision of the tumor and local radiotherapy. Methods A case report. Results A 25-year-old woman presented with perianal pain with defecation and was diagnosed with perianal fistula. The anal ultrasound revealed a densification of the perianal fat. The patient was submitted to a biopsy that revealed a high grade aRMS, with poor differentiation, grade 3 (FNCLCC) with less than 5 mitosis. The MRI reported a perianal mass with sphincter involvement and 
extension to ischio-rectal fossae. The PET scan described, apart from the primary lesion, inguinal ganglion methastasis. The genetic study showed the rearrangement of FOXO1 gene. Considering this, the patient was submitted to primary chemotherapy. Subsequently, the patient repeated the PET scan and there was no evidence of malignancy. The case was discussed in a multidisciplinary consultation and the patient was submitted to extended excision of the residual tumor via Kraske incision and inguinal linfadenectomy. The pathological analysis described a residual tumor with free surgical margins and linfadenectomy specimen did not have malignancy involvement. Afterwards, the patient was proposed to adjuvant radiotherapy. However, three months after the surgery the patient developed local relapse and diffuse methastasis. It was decided to submit the patient to palliative chemotherapy. Conclusion The perianal aRMS is a very aggressive tumor with poor prognosis and can present with disseminated disease at diagnosis. The surgical resection impact is not well clarified and the decision has to take into account the morbidity of the procedure (considering the quality of life after the surgery) and its efficiency in the control of the local disease. According to literature, surgery resection appears to have poor impact in overall survival, specially in high grade lesions.

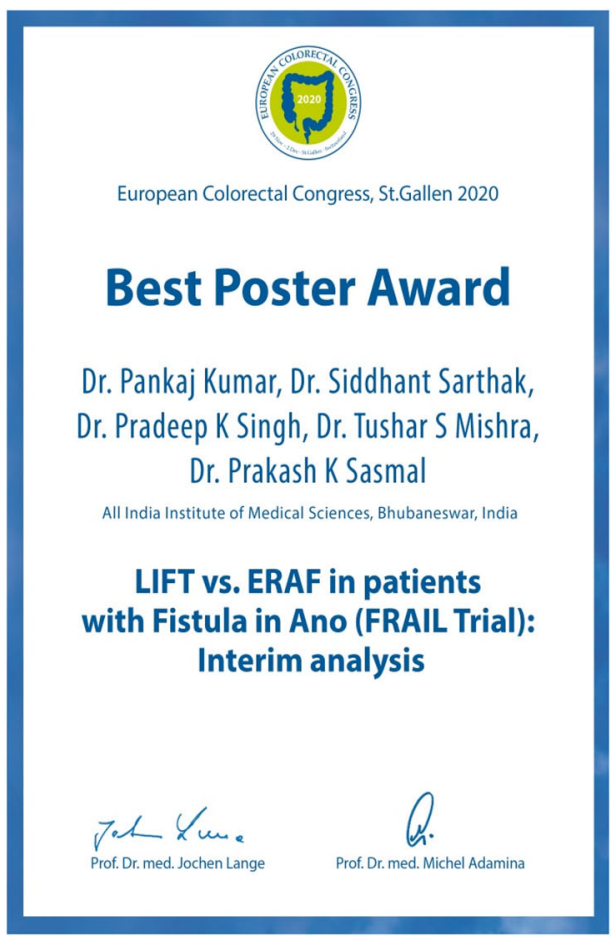

Dr. Pankaj Kumar from the All India Institute of Medical Sciences, Bhubaneswar, India was presented the Best Poster Award for his work on "LIFT vs. ERAF in patients with fistula in ano-an interim analysis of the FRAIL randomised controlled trial.

Publisher's Note Springer Nature remains neutral with regard to jurisdictional claims in published maps and institutional affiliations 